\title{
Dipole blockade in a cold Rydberg atomic sample
}

\author{
Daniel Comparat and Pierre Pillet
}

June 7, 2010

\begin{abstract}
We review here the studies performed about interactions in an assembly of cold Rydberg atoms. We focus more specially the review on the dipole-dipole interactions and on the effect of the dipole blockade in the laser Rydberg excitation, which offers attractive possibilities for quantum engineering. We present first the various interactions between Rydberg atoms. The laser Rydberg excitation of such an assembly is then described with the introduction of the dipole blockade phenomenon. We report recent experiments performed in this subject by starting with the case of a pair of atoms allowing the entanglement of the wave-functions of the atoms and opening a fascinating way for the realization of quantum bits and quantum gates. We consider then several works on the blockade effect in a large assembly of atoms for three different configurations: blockade through electric-field induced dipole, through Förster resonance and in van der Waals interaction. The properties of coherence and cooperativity are analyzed. Finally, we treat the role of dipole-dipole interactions between Rydberg atoms responsible for Penning ionization. The perturbation of the dipole blockade by ions and the evolution of the Rydberg towards an ultracold plasma are discussed.
\end{abstract}

\section{Introduction to Rydberg atoms}

The field of cold Rydberg atoms or molecules knows today an intense international activity, motivated by both fundamental scientific research and applications. The main attractivity of Rydberg systems is linked to the existence of huge electric dipolar momenta, offering various possibilities such as manipulation of the Rydberg particles with small gradients of electric field [1], or control of interactions between two particles at a macroscopic distance [2. By using highly excited Rydberg gases with strong and long-range inter-particle interactions, many complex quantum systems can be achieved with a full control of the dynamics, at the crossing of different areas as solid state or plasma physics. The effects of the long-range dipole-dipole interactions between Rydberg atoms have been recently reviewed in [2. The Rydberg excitation offers efficient tools for a quantum engineering especially for the entanglement of neutral particles 
(see the review in reference [3]). A demonstrated example is the dipole blockade [4] of the laser excitation, effect on which is focused this review and which is the result of the long-range interatomic dipole-dipole interactions shifting the Rydberg energy levels and preventing their laser excitation.

In the eighties, the atomic highly excited or Rydberg states (with $n, \ell, m_{\ell}$ respectively principal, orbital angular and magnetic quantum numbers) were already extensively studied for their exaggerated properties, as for instance their giant size $(\sim 1 \mu \mathrm{m}$ for $n \sim 100)$ ranging as $n^{2}$ in atomic units (Bohr radius, $\left.a_{0}\right)$ [5]. Such states are so sensitive to weak perturbations that they offer the opportunity to explore phenomena inaccessible for other physical systems in laboratory experiments. Rydberg atoms have huge polarizabilities leading to extreme collisional properties of room temperature atoms, in particular, large cross sections and long interaction times.

The effect of the dipole-dipole interaction displays very clearly in the interactions between Rydberg atoms having high principal quantum number $n$, whose dipole moments scale as $n^{2}$. Due to the strong and long-range interactions, a Rydberg gas offers the opportunity for studying $N$-body, many-body cooperative or collective effects [6, 7, 8, 2]. A key point is that it is experimentally possible to tune, for instance by micro-wave or external electric fields, the Rydberg energy levels to achieve a resonant condition. One of the most interesting possibility, on which this review is focused on, is when the two Rydberg atoms exchange resonantly internal energy by making transitions between Rydberg levels in a $r_{1}+r_{2} \rightarrow r_{1}^{\prime}+r_{2}^{\prime}$ iso-energetic reaction. This configuration of the collisional process, a so called Förster resonance [9], has been first demonstrated by Thomas F. Gallagher's group (Virginia University, USA) [10, 11] in a thermal ensemble and later together with Pierre Pillet's group (Orsay, France) in a cold atomic gas 12 , 13.

We concentrate this review to the study of cold Rybderg gases. Such study started in Pierre Pillet and Thomas Gallagher's groups in 1998 with already signature of $N$-body effects in order to interpret the system as a frozen gas with properties quite similar to those of an artificial solid [13, 12]. The first modeling of cold dipolar interaction was thus based on the so called "frozen Rydberg gas" hypothesis [14]. However, it has very soon be recognized that a dipole gas does not stay a long time as a frozen gas. The whole dynamics of the system necessitates to take into account the dipole forces between close Rydberg atoms [15]. These forces are at the origin of the creation of ions by Penning ionization. An extreme and impressive effect of this dynamics is the ultra-cold plasma formation [16, 17, 18].

The dipole-dipole interaction between Rydberg atoms creates a shift depending on the interatomic distance, giving the asymptotic dependence of the molecular potential curves at large distance from which the forces derive. This energy shift can also allows to control the excitation of the neighboring atoms around a previously excited one, as proposed in 2000-2001 [19, 4]. The shift created by the dipole-dipole interactions allow for instance a single Rydberg excitation in a large atomic sample, creating a Fock state. The dipole blockade regime for two atoms have been recently observed in the collective single 
excitation of a pair of individually trapped atoms [20, 21] . Such a result opens the way to the control of few-atom sample. Several previous studies in manyatom sample have displayed a large variety of phenomena: absorption, emission or ionization by black-body radiation, cooperative spontaneous emission with limited superradiance, collective excitation, many body interactions, dynamical dipolar forces, excitation transfer, dipole orientation effects, fine or hyperfine atomic effects, random position of atoms or ions effects in ultracold plasmas... Finally, with the great experimental control obtained, a many-atomic sample can probably be employed as quantum simulator to answer some challenging open questions of condensed matter [22, 23].

The paper is organized as following. In the section 2, we recall a few of the main characteristics of Rydberg atoms. In section 3 we discuss the RydbergRydberg interactions and more precisely the dipole-dipole ones. The case of the retardation effects is described carefully. In the section 4, we expose a first consequence of the dipole-dipole interaction, the dipole blockade of the Rydberg laser excitation of cold atoms. We also discuss recent experiments using simply two atoms, and succeeding to entangle them through a collective excitation in the blockade regime. In section 5 we detail the condition for the observation of the blockade in the case of a large sample in the configurations of the van der Waals interaction, of a permanent dipole induced by a static electric field, and of a Förster resonance. In the section 6 we discuss the limit of the picture of the frozen Rydberg gas, through the presence of the forces exerted between two close Rydberg atoms in dipole-dipole interaction. We report the possibility to control the attractive or repulsive character of these forces, for preventing the Penning ionization particularly important in the evolution of cold Rydberg gases toward ultracold plasmas. The section 7 is the conclusion.

\section{Properties of Rydberg atoms}

The properties of Rydberg atoms can be considered as really exaggerated compared to "classical" atoms [5. We have already mentionned the size, we underline the following characteristics

1. The binding energy is very small.

2. The radiative lifetime is very long.

3. The dipole matrix elements are big.

4. They are very sensitive to electric fields.

First, the binding energy $(\sim 300 \mathrm{GHz}$ for $n \sim 100)$ is very small $E=$ $\frac{e^{2}}{a_{0}} \frac{m-m_{e}}{m} \frac{1}{2\left(n-\delta_{\ell}\right)^{2}}$, where $e^{2}=\frac{q_{e}^{2}}{4 \pi \varepsilon_{0}}, m_{e}$ is the electron mass, $m$ the mass of the atom, and $q_{e}$ the electron charge. $n^{*}=n-\delta_{\ell}$ is the effective quantum number where $\delta_{\ell}$ is called the quantum defect and depends mainly only of the orbital angular momentum $\ell$ of the valence electron. The low $\ell$ angular states 
orbits penetrate the core and are therefore the most perturbated ones with large quantum defect. For instance the $\ell=0$ ( $s$ states) have a quantum defect $\delta_{0}=4.052$ in the cesium case. Spin-orbit effect are still present, even at high $n$ values, e.g. a fine structure splitting on the order of $100 \mathrm{MHz}$ exists for $\mathrm{Rb}(p)$ levels at $n=100$ [24].

Second, the radiative lifetime is very long, on the order of several microseconds. A useful very simplified formula to estimate the lifetime is [25] $n^{3}(\ell+1 / 2)^{2} 10^{-10} \mathrm{~s}$. Note that the lifetime rapidly increases as $n$ or $\ell$ increases. This formula does not include the blackbody effect [26] which, for a temperature $T$, increase the decay rate by roughly $2 \times 10^{7} \frac{T(K)}{300 K} n^{-2}\left(s^{-1}\right)[27$.

Third, as mentioned in the introduction, the radial dipole matrix element $R_{n \ell}^{n^{\prime} \ell \pm 1}=\left\langle n \ell|r| n^{\prime} \ell \pm 1\right\rangle$ are big1, on the order of $a_{0} n^{2}$. A useful semi classical expression [28, 29] is:

$R_{n \ell}^{n^{\prime} \ell \pm 1} \approx \frac{a_{0}}{s} \frac{n_{c}^{5}}{\left(n^{*} n^{\prime *}\right)^{3 / 2}}\left[J_{-s}^{\prime}(e s) \pm \sqrt{e^{-2}-1}\left(J_{-s}(e s)-\frac{\sin (\pi s)}{\pi s}\right)+(1-e) \frac{\sin (\pi s)}{\pi}\right]$

where $s=n^{* *}-n^{*}, n_{c}^{3}=\frac{2\left(n^{*} n^{\prime *}\right)^{2}}{n^{*}+n^{\prime *}}, e^{2}=1-\left(\frac{\ell+\ell^{\prime}+1}{2 n_{c}}\right)^{2}$ and $J_{s}(z)$ is the Anger function ( $J_{s}^{\prime}$ its derivative). This expression is valid to high accuracy (below 5 percent) for low angular momentum $(\ell<9)$ states 30 . It is also correct when $n^{\prime *}=n^{*}(s \rightarrow 0)$ and extension to the continuum states is straightforward, the only change is on the normalization factor [31, 32. The main message is that the electric dipole transition matrix can acquire huge values, $\sim n^{2} q_{e} a_{0}$, where the atomic unit of electric dipole moment is $-q_{e} a_{0}=8.48 \times 10^{-30} \mathrm{C} . \mathrm{m}=2.54$ Debye.

Forth, the Rydberg states are very sensitive to electric fields. The system of the hydrogen atom in the presence of an electric field is well known to have analytical solution. The effect of the electric field is to lift the $\ell$-degeneracy of the $n$ manifold. In the case of alkali atoms, the behavior is not different for high angular momentum states, while the low ones with large quantum defect have a quadratic energy behavior versus the electric field. The standard way to calculate the external electric field effect, i.e. the effect of the $-\vec{\mu} . \vec{F}$ Stark Hamiltonian, where $\vec{\mu}=q_{e} \vec{r}$ is the dipole operator of one atom, is to follow the work in reference [33]. A typical result is shown in figure1. If a (external or due to ions) static electric field $\vec{F}$ is present the Rydberg states are mixed, creating a permanent dipole moment for the Rydberg atoms. Adding a small electric field is therefore a very simple way to control the Rydberg-Rydberg interaction.

For small dc electric fields, i.e. when the dipole coupling is of the same order as the energy difference of the nearest state with allowed dipolar transition, the Stark effect is quadratic and the shift can be calculated with a reasonable approximation in a two level model. As illustrative example, in the cesium, this case concern the $r=n \ell=n p$ state is mainly mixed with the $r^{\prime}=n^{\prime} \ell^{\prime}=(n-1) d$

\footnotetext{
${ }^{1}$ This has not to be confused with the reduced matrix element $\left\langle n \ell\|r\| n^{\prime} \ell \pm 1\right\rangle=$ $\sqrt{2 \ell^{\prime}+1} C_{\ell+10,10}^{\ell 0}\left\langle n \ell|r| n^{\prime} \ell \pm 1\right\rangle, C_{j_{1} m_{1}, j_{2} m_{2}}^{j m}$ being the standard Clebch-Gordan coefficient.
}

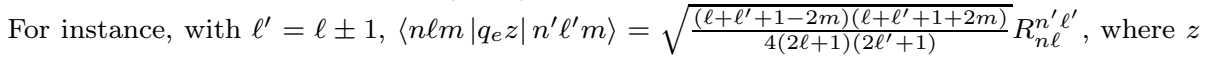
is the coordinate along the quantization axis. 


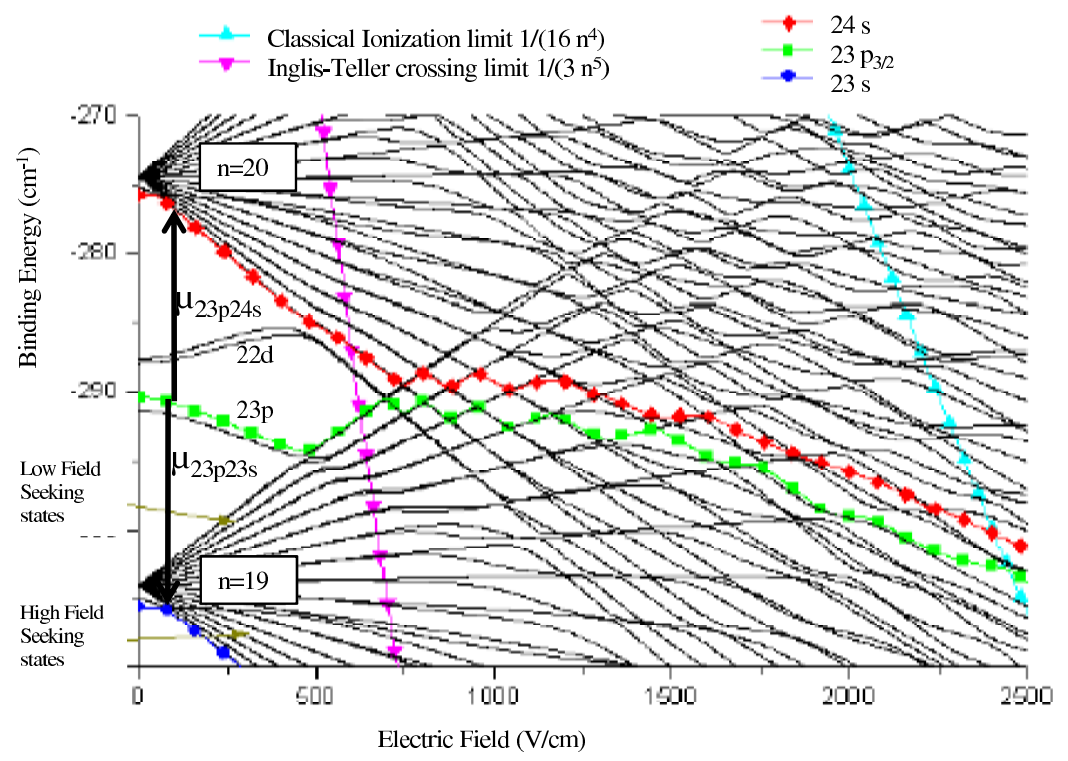

Figure 1: Energy levels of the cesium Rydberg state $n \ell j m_{j}$ with $\left|m_{j}\right|=1 / 2$ versus the electric field. The fine structure of the $23 p$ level is visible. The Förster resonance $23 p+23 p \rightarrow 23 s+24 s$ is resonant for a field of $\sim 80 \mathrm{~V} / \mathrm{cm}$.

state (the closest energetically nearby state with allowed dipole transition see figure 1). For simplicity of the writing we do not express here any dependence of $j$ the total angular momentum, neither of $m_{j}$ (or $m_{\ell}$ ) its projection along a chosen axis the external field or the internuclear axis. Depending on the experimental conditions (laser polarization mainly), the projection of the angular momentum can be controlled or not.

We denote by $\vec{\mu}_{r r^{\prime}}=\left\langle r|\vec{\mu}| r^{\prime}\right\rangle$ the transition dipole moment of the $|r\rangle \rightarrow\left|r^{\prime}\right\rangle$ transition. So the new eigenstates $\left(|r(\vec{F})\rangle,\left|r^{\prime}(\vec{F})\right\rangle\right)$ are given by the diagonalization of the Hamiltonian matrix:

$$
\left(\begin{array}{cc}
E_{r} & -\mu_{r r^{\prime}} F \\
-\mu_{r r^{\prime}} F & E_{r^{\prime}}
\end{array}\right)
$$

where $E_{r}$ and $E_{r^{\prime}}=E_{r}+\hbar \omega_{r r^{\prime}}$ are the energies of states $r$ and $r^{\prime}$ in absence of an electric field. We introduce the scale parameter $\vartheta$ characterizing the dipole coupling for each level $r$ by $\tan \vartheta=\frac{\mu_{r r^{\prime}} F}{\hbar \omega_{r r^{\prime}} / 2}$. The atom in state $|r(\vec{F})\rangle$ acquires a classical permanent electric dipole $\vec{\mu}_{r}(\vec{F})$ aligned along the local electric field $\vec{F}$ with value $\mu_{r}(F)=\left\langle r(\vec{F})\left|q_{e} z\right| r(\vec{F})\right\rangle=\mu_{r r^{\prime}} \sin \vartheta$. The resulting shift in energy for $r$ is $\hbar \Delta_{r}(\vec{F})=\frac{\hbar \omega_{r r^{\prime}}}{2}\left(1-\sqrt{1+\tan ^{2}(\vartheta)}\right)$.

In the case of non hydrogen atoms as alkali ones, another important feature of the effect of the electric field different from the hydrogen is the avoided crossings between the Stark levels of two different manifold. As a consequence the electric- 
field ionization of the Rydberg atoms depends on the risetime of the pulsed ionization field and correspond to the ionization classical limit. Consequently, an electric field of $3.21 \times\left(\frac{100}{n}\right)^{4} \mathrm{~V} / \mathrm{cm}$ is usually enough to ionize a Rydberg state.

\section{Interaction between two close Rydberg atoms}

Rydberg studies are often done in presence of an external electric field, either to ionize the atoms, to induce a permanent dipole moment or to study what is called a (resonant) excitation transfer.

Detail study of van der Waals and dipole-dipole energy shifts of pairs of interacting Rydberg atoms for different quantum numbers $n, l, j$, and $m_{j}$, taking into account a large number of perturbing states has been performed, and useful formula have been given in references [34, 35, 36, 24]. We would like here simply to introduce some of the key notions.

The electrostatic interaction hamiltonian between two atoms, the first one (core $A$ electron 1) and the second (core $B$ electron 2) separated by $\overrightarrow{R_{A B}}=R \vec{n}$ is, with obvious notations: $H=\frac{e^{2}}{R}-\frac{e^{2}}{r_{A 2}}-\frac{e^{2}}{r_{B 1}}+\frac{e^{2}}{r_{12}}$. Using $\left(\|\vec{R}+\vec{r}\|^{2}\right)^{-1 / 2}=$ $\frac{1}{R}\left(1-\frac{\vec{n} \cdot \vec{r}}{R}+\frac{3(\vec{n} \cdot \vec{r})^{2}-r^{2}}{2 R^{2}}+\cdots\right)$, the leading term in the asymptotic expansion (i.e. large $R$ ), is the so called dipole-dipole interaction and is found to be:

$$
H_{12}=\frac{e^{2}}{R^{3}}\left(\vec{r}_{A 1} \cdot \vec{r}_{B 2}-3\left(\vec{r}_{A 1} \cdot \vec{n}\right)\left(\vec{r}_{B 2} \cdot \vec{n}\right)\right)=\frac{\vec{\mu}_{1} \cdot \vec{\mu}_{2}-3\left(\vec{\mu}_{1} \cdot \vec{n}\right)\left(\vec{\mu}_{2} \cdot \vec{n}\right)}{4 \pi \varepsilon_{0} R^{3}} .
$$

Due to the long range interaction electron 1 is always close to core $A$ and electron 2 to core $B$ so there is not exchange interaction, i.e. no spatial overlap between the wave function of electron 1 and 2 . We thus think that there is no confusion in the notation $\vec{\mu}_{1}$ which is the dipole operator for the atom $A$.

Before using this hamiltonian to calculate potential energy curves between Rydberg atoms, we would like to study this hamiltonian in a (semi-)classical point of view which will allow us to use the retardated potentials.

In the appendix (section 9), see also [37, 38, we shall show the equivalence of this semi-classical approach, with the establishment of the master equation of the evolution of a pair of two-level atoms coupling with the electromagnetic field of the vacuum. The master equation is obtained in the Born-Markov approximation. It takes into account several effects blind to the semi-classical picture namely: the cooperative spontaneous emission or superradiance demonstrating the strong link between the two non-separable effects.

\subsection{Semi-classical derivation of the dipole-dipole hamilto- nian interaction}

\subsubsection{Instantaneous dipole-dipole interaction}

Classically speaking, a particle, such as one atom in the $|r(\vec{F})\rangle$ state, which possesses a permanent electric dipole moment, $\vec{\mu}$, generates an electric field. At a 
(vectorial) distance, $R \vec{n}$, the expression of the field is $\vec{E}=\frac{1}{4 \pi \varepsilon_{0}}[3(\vec{n} \cdot \vec{\mu}) \vec{n}-\vec{\mu}] \frac{1}{R^{3}}$. For two particles, in states $\left|r_{1}(\vec{F})\right\rangle$ and $\left|r_{2}(\vec{F})\right\rangle$, separated by the distance, $R \vec{n}$, the energy of the system is given by $V_{12}=-\vec{E}_{1} \cdot \vec{\mu}_{2}=-\vec{\mu}_{1} \cdot \vec{E}_{2}=\frac{\vec{\mu}_{1} \cdot \vec{\mu}_{2}-3\left(\vec{\mu}_{1} \cdot \vec{n}\right)\left(\vec{\mu}_{2} \cdot \vec{n}\right)}{4 \pi \varepsilon_{0} R^{3}}$. This results is exactly the quantum mechanical one given by Eq. (2), i.e. $V_{12}=\left\langle r_{1}(\vec{F}), r_{2}(\vec{F})\left|H_{12}\right| r_{1}(\vec{F}), r_{2}(\vec{F})\right\rangle$. More generally the hamiltonian $H_{12}$ is simply $V_{12}$ with the vectors dipoles $\vec{\mu}_{1}, \vec{\mu}_{2}$ becoming operators.

In the case where the imposed external field $\vec{F}$ is stronger than all other fields, such as the sum of the fields created by the dipoles, all dipoles are aligned along the same field $\vec{F}$. If we define the angle $\theta$ as $\cos \theta=(\vec{n} . \vec{F}) / F$ we thus have

$$
V_{12}=\frac{\mu_{1} \mu_{2}}{4 \pi \epsilon_{0} R^{3}}\left(1-3 \cos ^{2} \theta\right)
$$

This $1-3 \cos ^{2} \theta$ angular dependence has been observed experimentally by the Noel's group in [39] using a quasi one dimensional cylindrical atomic sample which could be rotated with respect to the external electric field.

\subsubsection{Retardated dipole-dipole interaction}

In absence of electric field, the Rydberg atoms have no permanent electric dipole. However they can still interact through exchange of excitation corresponding to the existence of a transition dipole from state $|n \ell\rangle=|r\rangle$ to state $\left|n^{\prime} \ell^{\prime}\right\rangle=\left|r^{\prime}\right\rangle$; the typical example being the $n s \rightarrow n p$ transition of an alkali atom.

Classically speaking, the transition $r \rightarrow r^{\prime}$, with dipolar matrix element $\vec{\mu}$ and transition energy $\hbar \omega=\hbar \omega_{r r^{\prime}}=E_{r^{\prime}}-E_{r}$ corresponds, using the complex representation, to an oscillating electric dipole momentum, $\vec{\mu} \exp (-i \omega t)$. This dipole radiates an electromagnetic field, at a vectorial distance, $R \vec{n}, \vec{E}(t)=$ $\vec{E}(\vec{R}) \exp (-i \omega t)$, with

$$
\vec{E}(\vec{R})=\frac{\exp (i k R)}{4 \pi \varepsilon_{0}}\left\{\left[k^{2}(\vec{n} \times \vec{\mu}) \times \vec{n}\right] \frac{1}{R}+[3(\vec{n} . \vec{\mu}) \vec{n}-\vec{\mu}]\left(\frac{1}{R^{3}}-\frac{i k}{R^{2}}\right)\right\}
$$

where $k=\omega / c$ is the wave number of the light. Thus in the reaction $r_{1}+r_{2} \rightarrow$ $r_{1}^{\prime}+r_{2}^{\prime}$, the electromagnetic field $\vec{E}_{1}(t)=\vec{E}_{1}(\vec{R}) \exp \left(-i \omega_{r_{1} r_{1}^{\prime}} t\right)$, radiated by the atom 1 interacts with the dipole $\vec{\mu}_{2}(t)=\vec{\mu}_{2} \exp \left(-i \omega_{r_{2} r_{2}^{\prime}} t\right)$ to give the interaction energy $-\vec{E}_{1}(t) \cdot \vec{\mu}_{2}(t)=-\vec{E}_{1}(\vec{R}) \cdot \vec{\mu}_{2} \exp \left(-i\left(\omega_{r_{1} r_{1}^{\prime}}+\omega_{r_{2} r_{2}^{\prime}}\right) t\right)$. The phase indicates the energy mismatch for the transition and $-\vec{E}_{1}(R \vec{n}) \cdot \vec{\mu}_{2}$ is the energy in complex notation, so the (real) energy is given by

$$
\begin{aligned}
H_{12}= & \operatorname{Re}\left[-\vec{E}_{1}(\vec{R}) \cdot \vec{\mu}_{2}\right]=\frac{1}{4 \pi \varepsilon_{0}}\left\{\left[\vec{\mu}_{1} \cdot \vec{\mu}_{2}-3\left(\vec{n} \cdot \vec{\mu}_{1}\right)\left(\vec{n} \cdot \vec{\mu}_{2}\right)\right]\left(\frac{\cos (k R)}{R^{3}}+\frac{k \sin (k R)}{R^{2}}\right)(\text { () })\right. \\
& {\left.\left[\vec{\mu}_{1} \cdot \vec{\mu}_{2}-\left(\vec{n} \cdot \vec{\mu}_{1}\right)\left(\vec{n} \cdot \vec{\mu}_{2}\right)\right] \frac{k^{2} \cos (k R)}{R}\right\} }
\end{aligned}
$$

where $k=k_{1}=\omega_{r_{1} r_{1}^{\prime}} / c$ is the wave number of the light. We could have also calculate the energy by using $\operatorname{Re}\left[-\vec{\mu}_{1} \cdot \vec{E}_{2}\right]$, which differs at first glance because 
$k$ would have been $k_{2}=\omega_{r_{2} r_{2}^{\prime}} / c$. But, due to energy conservation $\omega_{r_{1} r_{1}^{\prime}}+\omega_{r_{2} r_{2}^{\prime}} \sim$ 0 , only near resonant term would contribute in the final evaluation so $k_{1} \sim-k_{2}$. We note $\lambda=2 \pi /|k|$ the wavelength of the transition.

For a short distance, $R<\lambda=\lambda / 2 \pi$, the hamiltonian corresponds essentially to the electrostatic dipole-dipole interaction calculated previously in Eq. (2), and which can thus be interpreted as an exchange of excitation between the two atoms (as an exchange of a virtual photon).

For a large distance, $R>\lambda$, only the term in $1 / R$ of the radiated electromagnetic field is important.

It is interesting to consider the order of magnitude of the different parameters in the case of the electric-dipolar transition $n p \longrightarrow n s$ of an alkali atom. The difference between the quantum defects of the $s$ and $p$ states for any alkali atom (except lithium) is 0.5 . The wave-length associated to the transition is therefore $\lambda \sim 9 \mathrm{~cm}$ (resp. 2, 0.6, 0.25, 0.07 ) and $\lambda=\lambda / 2 \pi \sim 1.5 \mathrm{~cm}$ (resp. 0.3, 0.09,0.04, 0.01 ) for $n \sim 100$ (resp. $60,40,30,20$ ). These values should to be compared to the size of the cold atomic sample usually of the order of $0.1 \mathrm{~cm}$, which exceeds $\lambda$ for $n>22$ and $\lambda$ for $n>40$. At a distance of $1 \mu \mathrm{m}$, the dipole-dipole coupling is of the order of $80 \mathrm{GHz}$ (resp. 10, 2, 0.65, 0.13) for $n \sim 100$ (resp. 60, 40, 30, $20)$.

Finally, to have the quantum mechanical equivalent of Eq. (5) we have to proceed as previously, i.e. to replace the dipole vectors by operators. For the atom $i, \vec{\mu}_{i}^{r_{i} r_{i}^{\prime}}=\left\langle r_{i}|\vec{d}| r_{i}^{\prime}\right\rangle\left|r_{i}^{\prime}\right\rangle\left\langle r_{i}\right|$ is the dipolar operator associated to the transition $r_{i} \rightarrow r_{i}^{\prime}$ of energy $\hbar \omega_{r_{i} r_{i}^{\prime}}=E_{r_{i}^{\prime}}-E_{r_{i}}$. If we consider the reaction $r_{1}+r_{2} \leftrightarrow r_{1}^{\prime}+r_{2}^{\prime}$, such as the $41 d+49 s \rightarrow 42 p+49 p$ almost resonant reaction in rubidium (see figure 17). The total hamiltonian $H=H_{1+2}$ would contain the non interacting hamiltonian $H_{1}$ and $H_{2}$ and hamiltonian containing all the near resonant interactions $H_{12}$. The reaction $r_{1}^{\prime}+r_{2} \leftrightarrow r_{1}+r_{2}^{\prime}$ is not included because being out of resonance. Finally,

$$
\begin{aligned}
H_{1+2}= & \hbar \omega_{r_{1}}\left|r_{1}\right\rangle\left\langle r_{1}\left|+\hbar \omega_{r_{1}^{\prime}}\right| r_{1}^{\prime}\right\rangle\left\langle r_{1}^{\prime}\left|+\hbar \omega_{r_{2}}\right| r_{2}\right\rangle\left\langle r_{2}\left|+\hbar \omega_{r_{2}^{\prime}}\right| r_{2}^{\prime}\right\rangle\left\langle r_{2}^{\prime}\right|+ \\
& H_{12}^{r_{1} r_{1}^{\prime} ; r_{2} r_{2}^{\prime}}+H_{12}^{r_{1}^{\prime} r_{1} ; r_{2}^{\prime} r_{2}}
\end{aligned}
$$

where for instance $H_{12}^{r_{1} r_{1}^{\prime} ; r_{2} r_{2}^{\prime}}$ being the hamiltonian given by Eq. (5) where $\vec{\mu}_{1}$, being replace by $\vec{\mu}_{1}^{r_{1} r_{1}^{\prime}}, \vec{\mu}_{2}$, being replace by $\vec{\mu}_{2}^{r_{2} r_{2}^{\prime}}$ and $k=\omega_{r_{1} r_{1}^{\prime}} / c \sim-\omega_{r_{2} r_{2}^{\prime}} / c$.

\subsection{Migration and Förster vision}

The dipole-dipole coupling allows a pair of Rydberg atoms to exchange the excitation in the reaction $n p+n s \leftrightarrow n s+n p$. Independently of the external field it is an exactly resonant transition which can lead to a diffusion of the $n p$ excitation if surrounded by $n s$ atoms [40, it is sometimes calls migration reaction. Analogy can be made with excitation diffusion of excitons [41] or spin glasses systems [42]. Such coupling necessitates nevertheless to prepare the atoms in different states. 
Another configuration based on transition dipoles is the Förster resonance one where two atoms exchange internal energy in dipole-dipole interaction, corresponding to different dipole transitions. As simple illustrative example (see Figure 19, let us consider the cesium case and the excitation of the $n p$ states in an electric field. By Stark effect, the $n p$ energy level can be put midway between the energy of the $n s$ and $(n+1) s$ states. So that a reaction of the type $n p+n p \leftrightarrow n s+(n+1) s$ is resonant (i.e. does not require any energy input). The name of "Förster resonance" has been given by Thad Walker and Mark Saffman in the reference2 [9] by analogy with the FRET (Förster Resonant Energy Transfer) process present in biology [43. The FRET occurs when the dipolar interaction between two molecules allows excitation transfer between one molecule to the other one which then becomes fluorescent. The resonance condition comes form the fact at a given distance the dipolar interaction match the energy difference of the two molecular systems opening the reaction. The terminology "Förster resonance" does not seems to be fully defined but is usually used when the two initial states are the same i.e. for reactions of the type $n \ell+n \ell \leftrightarrow n_{1} \ell_{1}+n_{2} \ell_{2}$ which are listed for several atoms in reference [24]. This choice comes because most experiments use a single atomic species (homonuclear case, $A=B$ ) excited towards the same Rydberg level. However, to keep the spirit of the FRET transfer it is probably better to extend the term "Förster" resonance to any type of resonance which, contrary to the migration reaction, are not always exactly resonant such in as the rubidium $41 d+49 s \rightarrow 42 p+49 p$ reaction.

\subsection{Frontier of the long range van der Waals interaction between Rydberg atoms}

The dipole-dipole hamiltonian $H_{12}$ given by Eq. (5) or more simply by the non retardated Eq. (2) couples a Rybderg pairs of atoms $\left|r_{1}\right\rangle_{A}\left|r_{2}\right\rangle_{B}=\left|r_{1}, r_{2}\right\rangle$ towards other pairs $\left|r_{1}^{\prime}, r_{2}^{\prime}\right\rangle$ creating, in function of the internuclear distance $R$ between the two atoms the potential curves. In presence of electric field we should use the $|r(F)\rangle$ states, but to simplify the notations we study the case of the absence of an electric field where there is no permanent dipole moment $(\vartheta=0)$. The hamiltonian describing the dipole-dipole interaction, in the atomic bases $\left|r_{1}^{\prime}, r_{2}^{\prime}\right\rangle$ and $\left|r_{1}, r_{2}\right\rangle$ is: $\left(\begin{array}{cc}\hbar \Delta & V \\ V^{\dagger} & 0\end{array}\right)$ where we have noted the dipoledipole coupling $V=\left\langle r_{1}, r_{2}\left|H_{12}\right| r_{1}^{\prime}, r_{2}^{\prime}\right\rangle$ and $\hbar \Delta=\left(E_{r_{1}^{\prime}}+E_{r_{2}^{\prime}}\right)-\left(E_{r_{1}}+E_{r_{2}}\right)$ the Förster energy mismatch. $V$ is basically $C_{3} / R^{3}$ with $C_{3}$ being $e^{2} R_{n_{1} \ell_{1}^{\prime} \ell_{1}^{\prime}}^{n_{1}^{\prime}} R_{n_{2} \ell_{2}^{\prime} \ell_{2}^{\prime}}$ times a coefficient (called $\sqrt{\mathcal{D}}$ in [24]) depending on angular (Clebsch-Gordan) coefficients. We do not resolve the $m_{j}$ projection so $V$, or $C_{3}$, is an operator in this subspace. The same block matrix representation can be used in Eq. (11), and results are generally valid using this block representation. We note $\left|\widetilde{r_{1}, r_{2}}\right\rangle$ and $\left|\widetilde{r_{1}^{\prime}, r_{2}^{\prime}}\right\rangle$ the new eigenstates. As an example we show in Fig. 2 the

\footnotetext{
${ }^{2}$ Despite the typographical error in the name "Förster" in the title.
} 


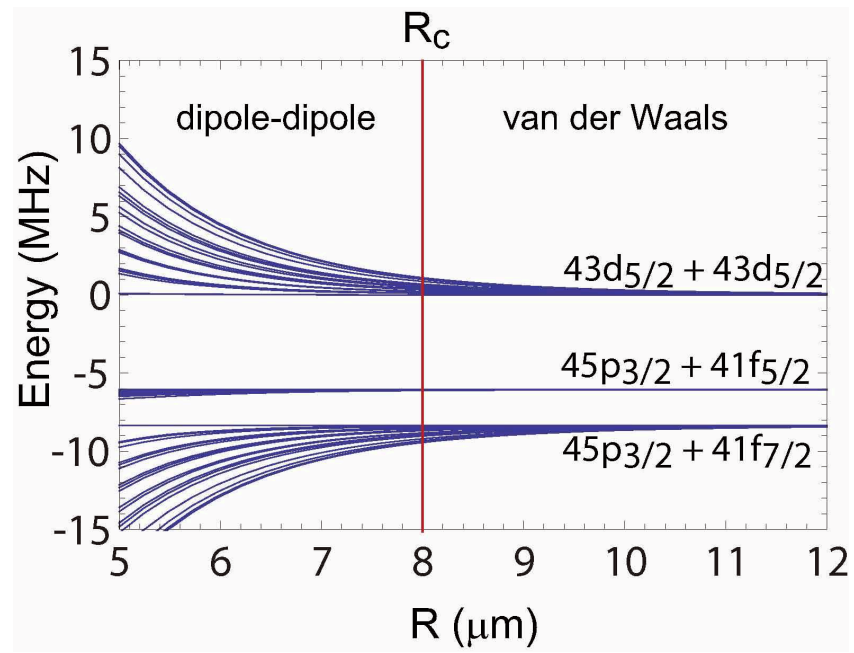

Figure 2: Potential energies for the interaction channel $43 d_{5 / 2}+43 d_{5 / 2} \rightarrow$ $45 p_{3 / 2}+41 f$ in rubidium Rydberg atoms. The cutoff radius $R_{c}=R_{v d W}$ represents the distance scale for the transition from resonant dipole-dipole to van der Waals behavior. From [3].

$43 d_{5 / 2}+43 d_{5 / 2}$ rubidium potential curves. The two $(j=5 / 2,7 / 2)$ nearby $f$ states interact strongly with the $43 d_{5 / 2}$ state. There are states with extremely small $C_{3}$, resulting in eigenstates that are nearly flat in energy and that would make difficult any blockade experiment.

The $r_{1}+r_{2}$ energy shift, which constitutes the $R$-dependent potential curves between the atoms, is given by the equation $\hbar \widetilde{\Delta}(R)=\frac{\hbar \Delta}{2}-\operatorname{sign}(\hbar \Delta) \sqrt{\frac{\hbar \Delta^{2}}{4}+\frac{C_{3}^{2}}{R^{6}}}$ where $\frac{C_{3}^{2}}{R^{6}} V^{\dagger} V=\left[24\right.$. Finally, the component of the new eigenstate $\left|\widetilde{r_{1}, r_{2}}\right\rangle$ on the states $\left|r_{1}, r_{2}\right\rangle$ and $\left|r_{1}^{\prime}, r_{2}^{\prime}\right\rangle$ are in ratio $\frac{V}{\hbar \Delta-\hbar \Delta}$.

We can define a cross-over distance, sometimes called van der Waals radius, $R_{v d W}$ via $\hbar \Delta=C_{3} / R_{v d W}^{3} . R_{v d W}$ denotes the region where the energies transition from the van der Waals to the resonant dipole-dipole form.

At small distances, $R \ll R_{v d W}$ the energy shift verifies $\hbar \widetilde{\Delta} \approx V=C_{3} / R^{3}$ which is the largest possible interaction energy between two Rydberg atoms. For instance, in the cesium case when the two atoms are in state $\left|r_{1}, r_{2}\right\rangle=$ $|n p, n p\rangle$, they interact strongly with the symmetric state $\left(\left|r_{1}^{\prime}, r_{2}^{\prime}\right\rangle=(\mid n s,(n+\right.$ $1) s\rangle+|(n+1) s, n s\rangle) / \sqrt{2}$ and not with the antisymmetric one $(|n s,(n+1) s\rangle-$ $|(n+1) s, n s\rangle) / \sqrt{2}$. This which leads to symmetric energy shifts $\mathrm{E}= \pm C_{3} / R^{3}$, corresponding to the symmetric and antisymmetric state

$$
[|n p, n p\rangle \pm(|n s,(n+1) s\rangle+|(n+1) s, n s\rangle) / \sqrt{2}] / \sqrt{2} .
$$

It is interesting to notice that the two atoms are naturally entangled in the Hund's base state $\left(\left|r_{1}, r_{2}\right\rangle+\left|r_{1}^{\prime}, r_{2}^{\prime}\right\rangle\right) / \sqrt{2}$ by the Förster coupling. This has to 
be compare to the entanglement created by the migration reaction $r_{1}+r_{2} \rightarrow$ $r_{2}+r_{1}$ in the homonuclear case where the best base to treat the problem is $\left(\left|r_{1}, r_{2}\right\rangle \pm\left|r_{2} r_{1}\right\rangle\right) / \sqrt{2}$.

At large distances $R \gg R_{v d W}$, the perturbated levels $r_{1}^{\prime}+r_{2}^{\prime}$ are far away (in energy compare to the coupling $V$ ) of the $r_{1}+r_{2}$ ones. When only one nearby energy level dominates, the energy shift expression becomes $\Delta E \approx \frac{\left(C_{3} / R^{3}\right)^{2}}{\hbar \Delta}=$ $C_{6} / R^{6}$. When no single $\left|r_{1}^{\prime} r_{2}^{\prime}\right\rangle$ state dominates the energy level shift of the $r_{1}, r_{2}$ state, this shift can still be calculated by using the second order perturbation theory: $\hbar \widetilde{\Delta}=\sum_{r_{1}^{\prime}, r_{2}^{\prime}} \frac{\left|\left\langle r_{1} r_{2}\left|H_{12}\right| r_{1}^{\prime} r_{2}^{\prime}\right\rangle\right|^{2}}{\left(E_{r_{1}^{\prime}}+E_{r_{2}^{\prime}}\right)-\left(E_{r_{1}}+E_{r_{2}}\right)}$.

Concerning the nomenclature some texts mean by van der Waals only the attractive forces and then sometimes distinguish van der Waals-Keesom, van der Waals-Debye, and van der Waals-London; others used "van der Waals" terms to design any kind of interactions repulsive or attractive ones. In this text we shall distinguish between effect calculated by first order perturbation theory and effect calculated using second order perturbation theory.

The first order perturbation theory, which creates interaction depening as $1 / R^{3}$ where $R$ is the interatomic distance, can be used when permanent dipole moment (i.e. Keesom's type) or near resonant transition dipole moment (Förster's type) exists. This is the case for states $n \ell+n^{\prime}(\ell \pm 1)$ state in homonuclear atoms $(A=B)$. Because of the $A(n s)+B(n p) \rightarrow A(n p)+B(n s)$ resonant migration transition $n s+n p$ (i.e. one atom in $n s$ level and the other in $n p$ level) present a $1 / R^{3}$ potential curve. This is the levels used in light-assisted collisions or photoassociation [44, 45].

The second order perturbation theory is used in other cases, i.e. when only induced dipole (London's type) moment are considered. It is thus mainly used in zero electric field, and the interaction energy has a $1 / R^{6}$ dependence as for the $n s+n s$ potential curves. We shall not consider here more complex interactions, i.e. higher order terms in the asymptotic expansion, such as quadrupolar one, because they are usually negligible. For instance interaction in $n p+n p$ state is of the $C_{5} / R^{5}+C_{6} / R^{6}$ form. However the $C_{5} / R^{5}$ quadrupolar term is usually small compare to the van der Waals $C_{6} / R^{6}$ one [24].

As the dipoles scales as $n^{2}$ the $C_{3}$ coefficient present a rapid $n^{4}$ scaling, and because $\hbar \Delta$ scales as the level spacing i.e. as $n^{-3}$, the $C_{6}$ coefficient present a rapid $n^{11}$ scaling.

\section{Conditional and collective laser Rydberg-excitation in the two-atoms blockade regime}

Interaction between cold atoms have been discussed several times in the context of quantum computation [46, 19, 4, 47. Since 2001, a large interest for the Rydberg atoms arise due to two theoretical propositions by Zoller's group [19, 4 ] intended to realize a fast quantum gate using ultracold atoms excited in Rydberg states. Indeed, the basics of a quantum gate is the control of a (quantum-)bit by another. This is exactly what was proposed in these references, and is described 
on the figure 3 the Rydberg excitation of one atom depends of the excitation of the first one due to the fact that the energy level is affected by the dipole-dipole interaction: this is the so called "dipole blockade" effect. Before considering the laser Rydberg-excitation of a large ensemble of cold atoms, we would like to introduce the interaction of two Rydberg atoms under a laser field excitation.

We would then described briefly experiments that have performed by Antoine Browaeys and Philippe Grangier's group (Institut d'Optique Graduate School, Palaiseau, France), in collaboration with our group, and by Mark Saffman and Thad Walker's group (University of Wisconsin, USA). Both group uses very small dipole trap to initially confine small number, ultimately single atoms [48, 49]. The two experimental setups are similar (even if the dipole trap is smaller in the French setup ensuring single atom trapping) and the distance between the two traps can typically be varied between 3 and $20 \mu \mathrm{m}$. Several Rydberg excitation state have been used $43 d_{5 / 2}, 58 d_{3 / 2}$, for their near resonant Förster properties at zero field, or $79 d_{5 / 2}, 90 d_{5 / 2}, 97 d_{5 / 2}$ for their stronger and stronger van der Waals interaction strength [3].

\subsection{Principle of the Rydberg blockade}
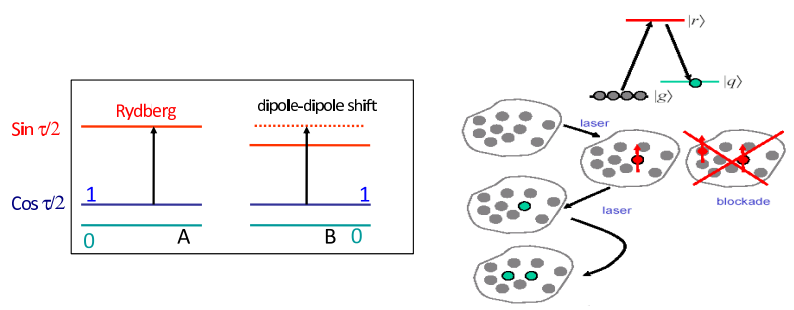

Figure 3: Application of the dipole blockade between two atoms. Left: the shift created by the dipole-dipole interaction allow to control the excitation of the second atom $B$ by the first one $A$ by preventing its excitation when the first atom $A$ has been excited. Right: when generalized to a larger atomic sample, the shift created by the dipole-dipole interaction allow a single excitation creating a Fock state. The short lived Rydberg Fock state can then be transfer by laser to a more stable one.

In the dipole blockade [4 of the laser excitation of cold Rydberg atoms the ground state $|g\rangle$ and the Rydberg state $|r\rangle$ of each atom are coupled by a laser with a Rabi frequency $\Omega$. When one atom is excited in a Rydberg state, the dipole-dipole interaction shifts the resonance and prevent the laser excitation of the second as explained also in figure 8 a). More precisely, when the two atoms are in state $|r, r\rangle$, they interact strongly which leads to symmetric energy shifts $E= \pm C_{3} / R^{3}$. When the interaction energy becomes larger than both the Rabi frequency and the laser resolution, the laser is out of resonance with the transition coupling the singly with doubly excited state, and only one atom at a time can be transferred to the Rydberg state. 


\subsection{Many photon laser excitation of two atoms}

The ground state - Rydberg transition is usually in the UV region so experimentalists usually prefer to excite Rydberg state via an intermediate level. Before describing the experiments, it is important to know if a more accurate treatment than "forgetting" about this intermediate state, to recover the previous 2 level $(g, r)$ picture, is needed or not. This procedure has been studied in detail by the Dresden group [50] in the rubidium $g=5 s \rightarrow 5 p \rightarrow r$ scheme. The relaxation of the populations and of coherences have to be taken into account using Optical Bloch Equations. The authors show, see Figure 4, that if it is possible to adiabatically eliminate the coherence in the equations to reach standard two level rate equation, the adiabatic elimination of the intermediate state has to be done with care. For instance, when the Autler-Townes splitting, of the intermediate step, are equal to the dipole-dipole Rydberg shift this increases the Rydberg excitation probability ( $n \sim 65$ case in Figure $4 \mathrm{f}$ ). as shown for $n \sim 65$ in Figure $4 \mathrm{f}$ ). This phenomenum has been called by the authors an "antiblockade" effect because it leads to an non so efficient blockade mechanism. This anti-blockade effect can not be predicted using simply the $(g, r)$ picture but is easily understand by coupling (dressing) by laser the ground state with the intermediate level. The anti-blockade arise when this Autler-Townes state is put into resonance with the shifted Rydberg-Rydberg level.

\subsection{Conditional excitation of a single Rydberg atoms}

We are going to treat theoretically two different cases: the conditional Rydberg excitation of one atom in presence a close neighbor Rydberg atom and the collective Rydberg excitation of a pair of atoms. The two situations refer to two different experiments: the conditional one addresses individually each atom by separate lasers, whereas the collective one addresses both atoms by the same laser. We treat here the most general case of a non-resonant Förster resonance. The total hamiltonian is $H_{1+2}$, cf Eq. (7), added to the laser interaction hamiltonian: $-\vec{\mu}_{1} \cdot \vec{E}_{L} \cos \left(\omega_{L} t-\vec{k} \cdot \vec{R}_{1}\right)$ for the atom 1 and $-\vec{\mu}_{2} \cdot \vec{E}_{L} \cos \left(\omega_{L} t-\vec{k} \cdot \vec{R}_{2}\right)$ for the atom 2 .

For a conditional excitation, we consider a one-photon excitation driving. The ground state $\left(g_{2}\right)$ of the second atom toward Rydberg state $\left|g_{2}\right\rangle \rightarrow\left|r_{2}\right\rangle$

transition with a Rabi frequency $\Omega=\left\langle g_{2}\left|-\vec{\mu}_{2} \cdot \vec{E}_{L}\right| r_{2}\right\rangle / \hbar$ and a laser detuning $\delta=\omega_{L}-\omega_{g_{2} r_{2}}$. The first atom is previously excited in a Rydberg state $r_{1}$. We have to consider the transition $\left|r_{1}, g_{2}\right\rangle \leftrightarrow\left|r_{1}, r_{2}\right\rangle$ and the Förster coupling $\left|r_{1}, r_{2}\right\rangle \leftrightarrow\left|r_{1}^{\prime}, r_{2}^{\prime}\right\rangle$ (strength $V$ ). We choose to decompose the wavefunction as:

$$
\psi(t)=a_{g}(t)\left|r_{1}, g_{2}\right\rangle+e^{i \vec{k} \cdot \vec{R}_{2}} e^{-i \omega_{L} t}\left[a_{r}(t)\left|r_{1}, r_{2}\right\rangle+a_{F}(t)\left|r_{1}^{\prime} r_{2}^{\prime}\right\rangle\right] .
$$



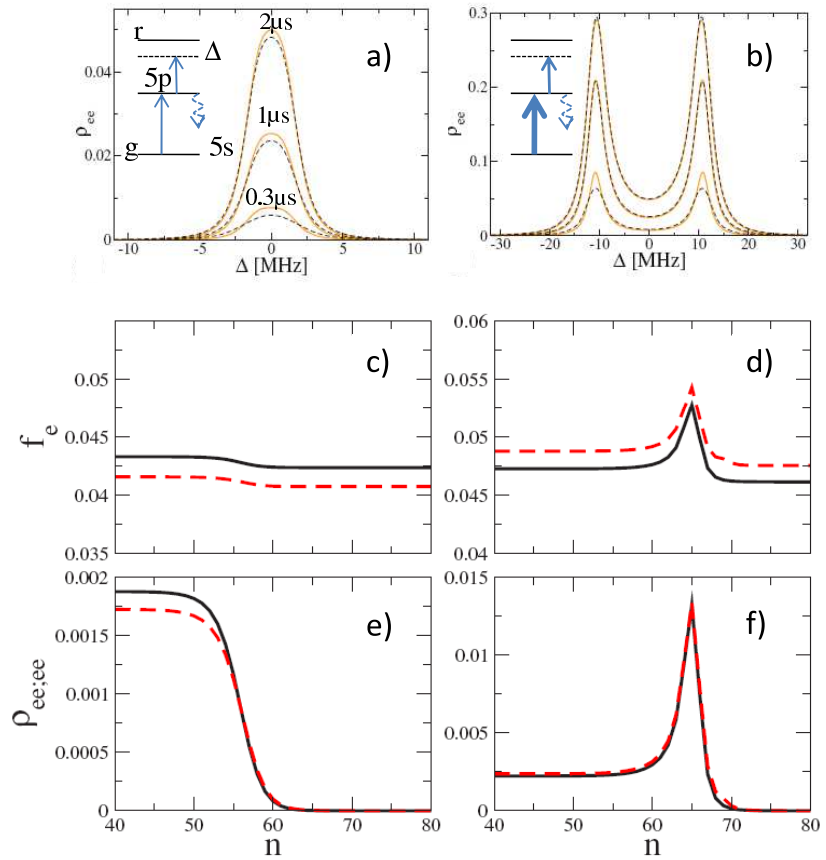

Figure 4: Population of the Rydberg level for the rubidium two-step excitation. Solid line is the result of the Rate Equation and dashed line of the full Optical Bloch Equation. Each column shows results with constant Rabi frequency, but the second column describes results when an higher laser intensity is used for the first excitation step, as schematically indicated in the inset of a) and b). a) and b) display the Rydberg excitation for a single atom (for different pulse length of $0.3,1.0$ and $2.0 \mu \mathrm{s}$. c)-f) results for two interacting atoms case (scaled to the case of two $n=48$ (s) atoms separated by $5 \mu \mathrm{m}$ ). $\rho_{e e}$ is the population of the Rydberg level, $f_{e}$ is the fraction of excited atoms, $\rho_{e e, e e}$ is the probability that both atoms are in the Rydberg state. The blockade effect is visible for $n>60$ in (e), and the anti-blockade is visible for $n \sim 65$ in (f). Adapted from [50].

The evolution of the system is given by (with energy origin for the state $\left(\left|r_{1}, r_{2}\right\rangle\right)$ :

$$
\begin{aligned}
i \frac{d a_{g}}{d t} & =\delta a_{g}+\frac{\Omega}{2} a_{r} \\
i \frac{d a_{r}}{d t} & =\frac{\Omega^{*}}{2} a_{g}+\left(V^{*} / \hbar\right) a_{F} \\
i \frac{d a_{F}}{d t} & =(V / \hbar) a_{r}+\Delta a_{F}
\end{aligned}
$$

Where we have performed the rotation wave approximation consisting to neglect the non resonant terms. We are not solving here the equation, but its resolution 


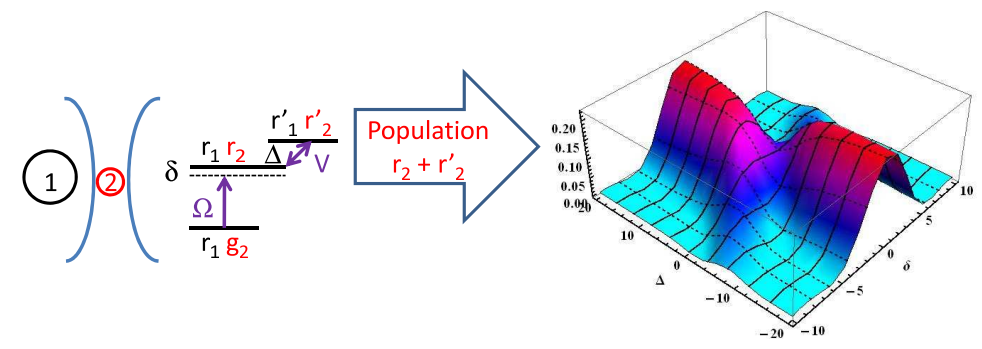

Figure 5: Calculations of the conditional excitation versus the laser-excitation, $\delta$, and the Förster resonances detuning $\Delta$. The calculation is performed at small time $\Omega t=1$ and in a strong coupling $(V=3 \hbar \Omega)$ regime. At Förster resonance, $\Delta=0$, we observe a blockade effect of the excitation at resonance $\delta=0$.
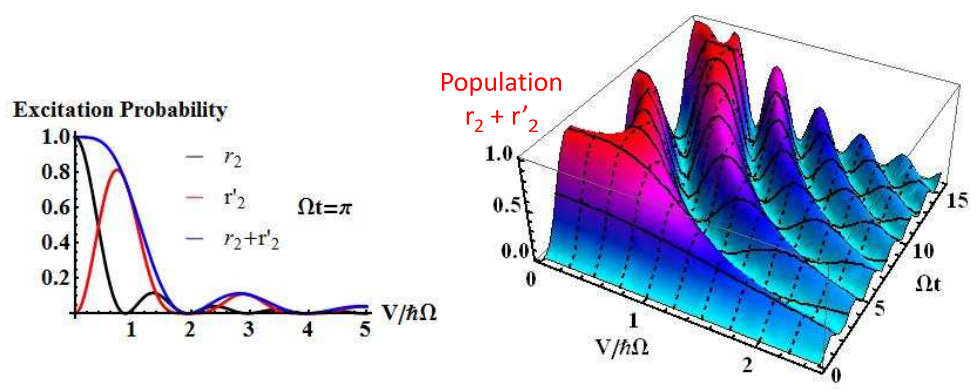

Figure 6: Conditional excitation of a second atom into a Rydberg state $\left|r_{2}\right\rangle$ when a neighboring atom is already in Rydberg state. Same notations as in figure 5 . Excitation probability in function of the strength of the dipole-dipole interaction $V$, of the interaction time $t$ (in Rabi $\Omega$-frequency units). Rabi oscillation are visible in the time evolution. The blockade of the excitation is obtained as soon as $V \gg \hbar \Omega$.

is easy, especially in the Förster resonance case of $\Delta=0$, if using the $\left|\widetilde{r_{1}, r_{2}}\right\rangle$ base.

The figure 5 shows in a perturbative regime for the excitation the probability of excitation versus laser excitation resonance, $\delta$, and the Förster resonance, $\Delta$. At Förster resonance, $\Delta=0$, we observe a blockade effect of the excitation at laser resonance $\delta=0$. This corresponding to the lift of the degeneracy between $\left|r_{1} r_{2}\right\rangle$ and $\left|r_{1}^{\prime} r_{2}^{\prime}\right\rangle$ state due to the dipole-dipole coupling. When scanning the laser detuning $\delta$ this leads also to a broadening, or even a splitting in our strong coupling case, of the Förster resonance.

The figure 6 shows, in a more detail way, the probability for the Rydberg excitation of a second atom versus the Förster coupling for a square pulse excitation. As visible in the case of a $\pi$ excitation $(\Omega t=\pi)$, for intermediate coupling $(V \simeq \Omega)$ a large amount of population are in the Förster Rydberg states $r_{1}^{\prime}$ and $r_{2}^{\prime}$. 


\subsection{Experimental realization of conditional Rydberg exci- tation}

As mentioned, previously, propositions to use pairwise controlled dipole-dipole interactions 46] as a very efficient realization of a quantum logic gate or to entangle neutral atoms has pushed experimentalist to realize the gedanken experiment proposed in reference 4 .

Rabi oscillation (previously observed by M. Weidemüller's group in an larger atomic sample [51]) has first been observed, by the Wisconsin team, in laser excitation of a single trapped atom. This has indicated that the system is not submitted to strong decoherence effects and has opened the way to further experiments [52]. The dipole blockade of the laser excitation, for a pair of individually trapped atoms, has indeed been observed recently by the two experimental groups [53, 20]. Two atoms are confined in two independent optical dipole traps, which are usually turned off during the excitation to avoid an extra light-shift. A successful excitation of an atom to the Rydberg state is detected through the loss of the atom when the dipole trap is turned back on, as atoms in the Rydberg state are not trapped in the tweezers. One of the key result is that single Rydberg-excited rubidium atom blocks excitation of a second atom located more than $10 \mu \mathrm{m}$ away (this so called blockade radius depends on the choice of the Rydberg state) has shown for instance in figure 7

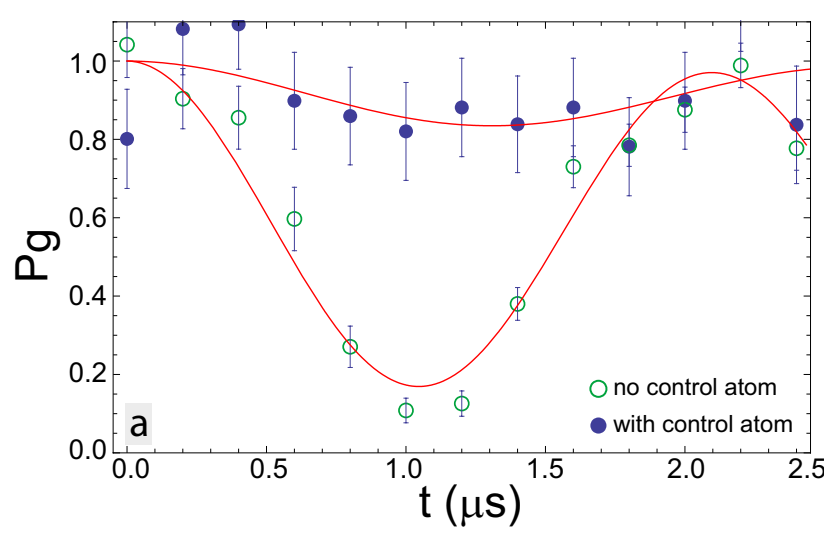

Figure 7: Single atom Rabi oscillation and evidence for blockade of the Rydberg excitation when a second atom is present. Experimental data for Rydberg excitation of the target atom with and without a second (control) atom present. From [53] 


\subsection{Experimental realization of collective excitation of a pair of Rydberg atoms}

The collective two-atom behavior has also been experimentally demonstrated with the excitation of an entangled state between the ground and Rydberg levels [20]. In this experiment both atoms are simultaneously illuminated by the excitation laser beams.

The principle is indicated in Figure 8 (a), when both atoms are simultaneously excited. A fundamental consequence of the blockade is that as any of the two atoms can carry the excitation, they end up in the entangled state $\left|\Psi_{+}\right\rangle=\frac{1}{\sqrt{2}}\left(|g, r\rangle e^{i k R_{2}}+|r, g\rangle e^{i k R_{1}}\right)$, where $R_{1}$ and $R_{2}$ are the position of the two atoms, and $k$ the wavevector of the excitation. The coupling from the two-atom state $|g, g\rangle$ toward the state $\left|\Psi_{+}\right\rangle$is $\sqrt{2} \Omega$, while the state $\left|\Psi_{-}\right\rangle=$ $\frac{1}{\sqrt{2}}\left(|g, r\rangle e^{i k R_{2}}-|r, g\rangle e^{i k R_{1}}\right)$ is not coupled with the ground state. In the blockade regime, the two atoms are therefore described by an effective two-level system involving collective states $|g, g\rangle$ toward $\left|\Psi_{+}\right\rangle$with a Rabi frequency $\sqrt{2} \Omega$.

The figure 8 (b) shows the experimental result where the Rydberg excitation is applied either to a single atom or to two neighboring atoms at a distance of $3.6 \mu \mathrm{m}$. The probability to excite both atoms in the Rydberg state is suppressed, as it is expected in the blockade regime. The probability to excite only one of the two atoms as a function of the duration of the excitation, can be compared with the probability to excite one atom inside a given trap when the other trap is empty. The two probabilities oscillate with different frequencies in ratio $1.38 \pm 0.03$ close to the expected value of $\sqrt{2}$. This result is the signature of the collective behavior of the two atoms and has been used to create the entanglement of two hyperfine ground state atoms by laser inducing the decay of the Rydberg state. The entangled state, reached with a fidelity of 0.75 , is generated in only $200 \mathrm{~ns}$ using pulsed two-photon excitation and has been quantify by applying global Raman rotations on both atoms [54. Finally the first demonstration of a CNOT gate, with fidelity of 0.73 , using neutral atoms have been achieved in Wisconsin [21].

Both the Palaiseau and Wisconsin experiments suffer from atoms losses during the gate operation and results have to be improved to reach the high fidelity results obtained with trapped ions. However these exciting recent experiments, pave the way for demonstrating more complex operations with several qubits [3]. Finally, such clean experiments performed in sample containing only few atoms have brought better understanding of the collective behavior as well as on the decoherence processes occurring in Rydberg assembly. Such, small atom number experiments are ideal to study of a Rydberg sample. Because a recent review article cover these subjects we shall not focus more on it here [3]. We simply give a simple theory able to describe the system 


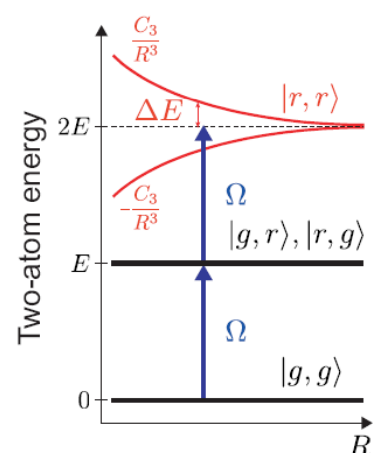

(a)

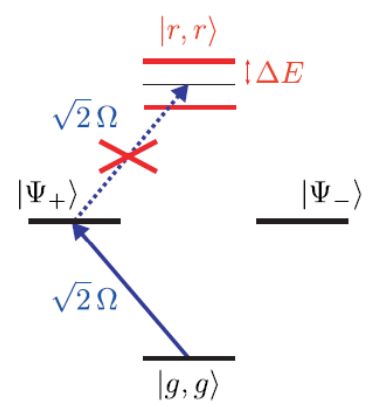

(b)

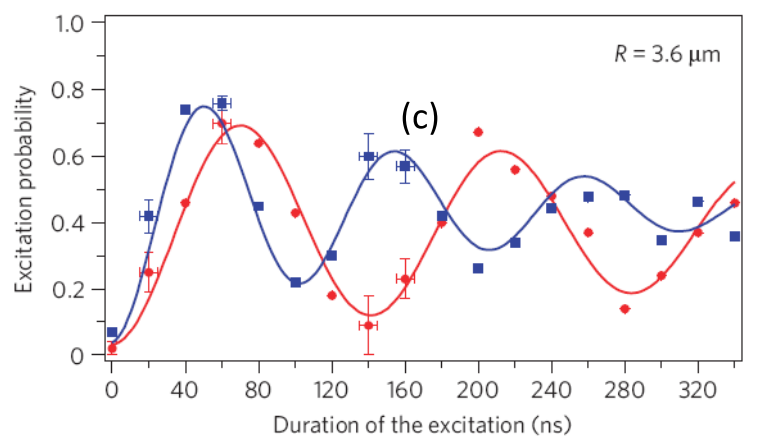

Figure 8: Principle and experimental realization of the Rydberg blockade. (a) Principle of the blockade between two atoms, in the regime of conditional excitation. When both atoms are simultaneously excited in the blockade regime, the symmetrical state $\left|\Psi_{+}\right\rangle$, described in the text, is only coupled to the ground state $|g, g\rangle$ with a strength $\sqrt{2} \Omega$ while the state $\left|\Psi_{-}\right\rangle$is not coupled by the laser to the states $|g, g\rangle$ and $|r, r\rangle$. c) Collective excitation of the two atoms separated by $3.6 \mu \mathrm{m}$. The circles represent the probability to excite one atom when the second atom is absent. The squares represent the probability to excite only one atom when the two atoms are trapped and are exposed to the same excitation pulse. From [20]

\subsection{Theory for collective excitation of a pair of Rydberg atoms}

The case of the collective excitation of the pair of atoms is a little bit different from the conditional excitation. Indeed, to realize a collective excitation both atoms should be undiscernable for the laser excitation, we call $|r\rangle$ the target Rydberg level and $|F\rangle$ the Förster state coupled to $|r, r\rangle$, for instance in the experiment described in figure $8,|r\rangle=|58 d\rangle$ and $|F\rangle=(|60 p, 56 f\rangle+|56 f, 60 p\rangle)$. 
A good basis to study the process is the Dicke states:

$$
\begin{array}{r}
|g, g\rangle \\
\left|\Psi_{+}\right\rangle=\frac{1}{\sqrt{2}}\left(|g, r\rangle e^{i k R_{2}}+|r, g\rangle e^{i k R_{1}}\right) \\
\left|\Psi_{-}\right\rangle=\frac{1}{\sqrt{2}}\left(|g, r\rangle e^{i k R_{2}}-|r, g\rangle e^{i k R_{1}}\right) \\
|r, r\rangle
\end{array}
$$

with the Förster state $|F\rangle$ added. We note that there is no laser coupling between the ground state $|g, g\rangle$ of the pair of atoms and the antisymmetrical state $|-\rangle$. So the wave-function for the pair of atoms is written as

$\psi(t)=b_{g}(t)|g, g\rangle+b_{+}(t) e^{-i \omega_{L} t}\left|\Psi_{+}\right\rangle+e^{-2 i \omega_{L} t} e^{i k R_{1}} e^{i k R_{2}}\left[b_{r}(t)|r, r\rangle+b_{F}(t)|F\rangle\right]$

The equations for the laser excitation are

$$
\begin{aligned}
i \frac{d b_{g}}{d t} & =2 \delta b_{g}+\frac{\Omega \sqrt{2}}{2} b_{+} \\
i \frac{d b_{+}}{d t} & =\frac{\Omega^{*} \sqrt{2}}{2} b_{g}+\delta b_{+}+\frac{\Omega \sqrt{2}}{2} b_{r} \\
i \frac{d b_{r}}{d t} & =\frac{\Omega^{*} \sqrt{2}}{2} b_{+}+\left(V^{*} / \hbar\right) b_{F} \\
i \frac{d b_{F}}{d t} & =(V / \hbar) b_{r}+\Delta b_{F}
\end{aligned}
$$

The coupling with the symmetrical one $|+\rangle$ is the Rabi frequency, $\Omega$, for the excitation of a single atom multiplied by a factor $\sqrt{2}$. The figure 9 shows at resonance laser $\delta=0$, and for a resonant Förster configuration $\Delta=0$, the transfer of populations for a pair of atoms in the Rydberg states out and in the blockade regime. We have represented: $\left|b_{+}\right|^{2}$ which represent the probability that one atoms is excited, $\left|b_{r}\right|^{2}+\left|b_{F}\right|^{2}$ that two atoms are excited and $\left|b_{F}\right|^{2}$ that the population is in the Förster state.

\section{Dipole-dipole interaction in a many atoms gas sample}

The dipole blockade effect can in principle be generalized to an assembly of $N$ atoms. However, the energy shift created by the dipole-dipole interactions creates, in a randomly distributed sample, a band of energy level and not anymore simply two levels as for the two atoms case. In simple case, such as in van der Waals one, the energy levels mainly depend on the number of excited Rydberg states and the principle of the blockade can be easily extended. However, in pure dipolar $\left(1 / R^{3}\right)$ cases the band structure can be very complex. Indeed, even in a simple three atomic system, T. Pohl and P. R. Berman [55] have shown 

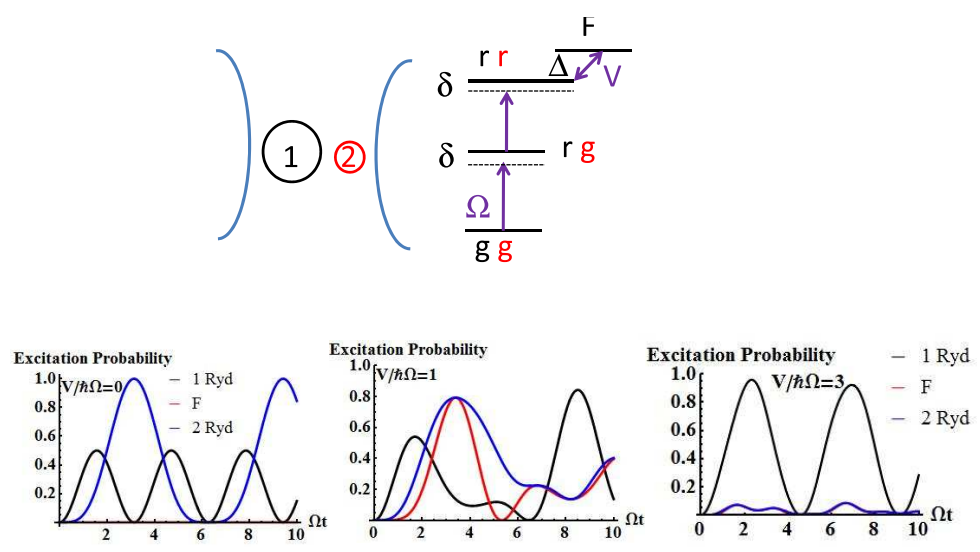

Figure 9: Collective excitation of two atoms, i.e. creation of a single but delocalized Rydberg excitation. The laser, of single atom Rabi frequency $\Omega$, is tuned at the resonance $\delta=0$, and the Rydberg state is energy shifted by a resonant Förster dipole-dipole interaction of strength $V=\left\langle F\left|H_{12}\right| r, r\right\rangle$ equals to 0,1 and 3 times $\hbar \Omega$. We present the probability that one atoms (number 1 or 2) is excited in the Rydberg state $|r\rangle$, that two atoms are excited but in the Förster state $|F\rangle$ and that the two atoms are excited in any Rydberg states $|r, r\rangle$ or $|F\rangle$. The blockade occurs when the Rydberg state is shifted more than the Rabi frequency i.e. for $V \gg \hbar \Omega$. Rabi oscillation are visible and, in the blockade regime, with a $\sqrt{2}$ speed enhancement for the collective excitation (single delocalized Rydberg excitation) compare to the single atom one.

that a noninteracting (so called zero energy) state exist even if strong two-body interaction exists. Existence of such zero states is very interesting to study but is problematic for blockade experiments because they could be accessed by many-photon excitation even when the two-photon excitation is well blockaded.

We are now going to describe experiments that have tried, and succeed, to observe the dipole- blockade in a many atoms system. In a simple picture, if the volume of the laser excitation is small enough, no second atom can be excited after the Rydberg excitation of a first one, producing an atomic ensemble in a singly excited collective state as pictured in Figure 3. Thus, the dipole-dipole interaction between Rydberg atoms should lead to a limitation of the number of excited atoms i.e. a partial, or local blockade of the excitation.

Depending on the size of the sample the blockade can be total (single excitation allowed) or limited. It may be useful to introduce the "blockade sphere" picture [56] which is especially well adapted to the quasi-isotropic van der Waals interaction. The excitation of a single Rydberg atom prohibits, via the blockade mechanism, subsequent excitations of other ground-state atoms within the radius $R_{b}$ of the blockade sphere. Since the $N_{b}$ atoms within the blockade sphere are indistinguishable, they behave as a "superatom" [57. The term "superatom" is given here by analogy with the superatom in clusters physics which is a clus- 
ter of atoms that exhibits some of the properties of elemental atom. Here the superatom interacts with the excitation light via a collective $\sqrt{N_{b}}$ enhancement of the Rabi frequency [58]. The dipole blockade effect implies that only a single atoms can be excited among $N_{b}$, into the state

$$
\left|\Psi_{+}\right\rangle=\frac{1}{\sqrt{N_{b}}} \sum_{i}\left|1(g), \cdots,(i-1)(g), i(r),(i+1)(g), \cdots, N_{b}(g)\right\rangle e^{i \vec{k} \cdot \vec{R}_{i}}
$$

, creating a faster (by a factor $\sqrt{N_{b}}$ ) Rabi oscillation of the one atom excitation compare to the Rabi oscillation of a single isolated atom. This behavior has indeed been observed on many atomic system by T. Pfau's team [59] before being very clearly observed in the case of $N_{b}=2$ by the Palaiseau group [20, 60].

\subsection{Saturation of the Van der waals Rydberg excitation}

In order to observe the blockade effect the standard pulsed dye laser spectroscopy is not accurate enough and a spectroscopic accuracy on the order of the dipolar interaction involved (typically $\sim 10-100 \mathrm{MHz}$ ) is required. In 2004, P. Gould and E. Eyler's group in Connecticut [56] uses a narrowband pulsed dye laser to realize the first demonstration of a partial van der Waals blockade as shown in figure 10 .

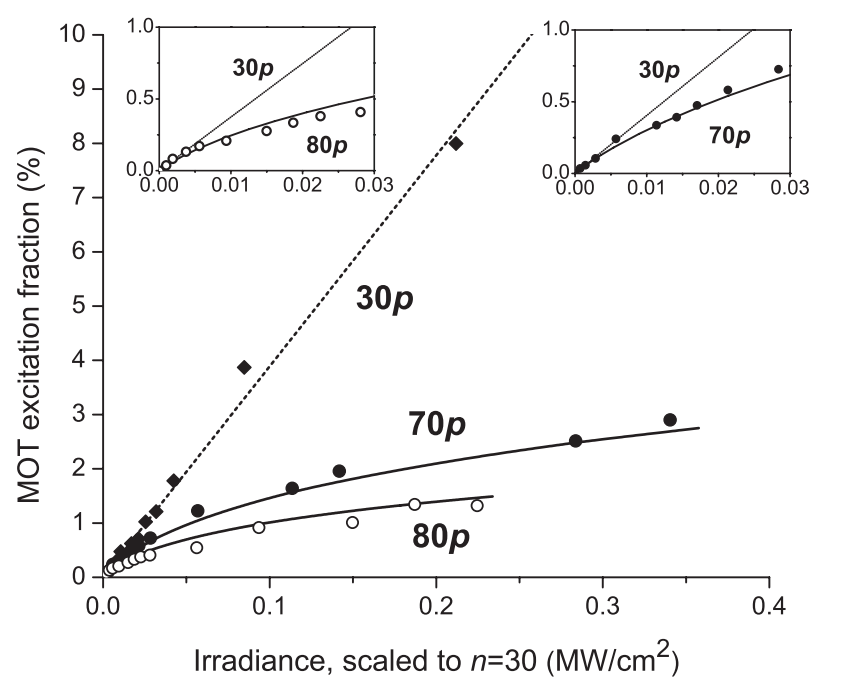

Figure 10: Van der Waals blockade of the excitation. Dependence of the Rydberg excitation fraction in function of the laser power exciting Rydberg state for several $n$ principle quantum numbers. For large $n$ the saturation of the Rydberg excitation clearly indicates a blockade effect. From [56]

However experiments using Continuous Wave (cw) lasers are the more numerous ones. The first one has been reported by Matthias Weidemüller's group 
in 2004 (Fribourg, Allemagne) [61]. The main difficulties of such cw experiments is that the presence of a single ion during the excitation can stop the excitation and then mimic the effect of the dipole blockade as probably observed in this first experiment [50, 62. Indeed, a single ion creates a spurious field of $150 \mathrm{mV} / \mathrm{cm}$ at a distance of $10 \mu \mathrm{m}$ which is roughly hundred times higher than the field created by the dipole of a $(n=50)$ Rydberg state. Such field could easily shifting energy levels by few $\mathrm{MHz}(300 \mathrm{MHz}$ for $n=50 \mathrm{Rb}$ atoms), i.e. larger than the laser linewidth creating a blockade of the excitation. Thus, to avoid any formation of ions during the excitation, the duration of the exciting laser light should be short enough. Another limitation of the blockade occurs for a broad-band or for a high-intensity excitation. In such cases, the suppression of the excitation is no longer expected, since pairs of close atoms can be excited out of resonance. However, despite all these difficulties, as we shall see several groups (including Weidemüller's one) have succeeded to observe the Rydberg blockade of the excitation.

As studied by Francis Robicheaux [63] and Hans Peter Büchler [64, and recently observed by the Stuttgart's group [65] (see figure 11), the number $N_{b}$ of blocked Rydberg atoms (total number of atoms divided by the number of excited Rydberg atoms) displays algebraic scaling laws, with a universal exponent, in function of the initial ground state atomic density $n_{g}$. This can be seen as a validation of the blockade radius picture. Indeed, in the van der Waals regime one simple scaling law is $N_{b} \sim\left(\frac{4 \pi}{3} n_{g} \sqrt{\frac{C_{6}}{\hbar \Omega}}\right)^{4 / 5}$. This is derived from the fact that the number of atoms blocked per excited atom is $N_{b} \sim n_{g} \frac{4 \pi}{3} R_{b}{ }^{3}$ (when the excited atom blocks all other atoms within the spherical volume of blockade radius $R_{b}$ ) and from $C_{6} / R_{b}{ }^{6} \sim \hbar \Omega \sqrt{N_{b}}$ because the blockade occurs when the detuning equals the excitation linewidth (i.e. the Rabi frequency for sufficiently narrow linewidth laser).
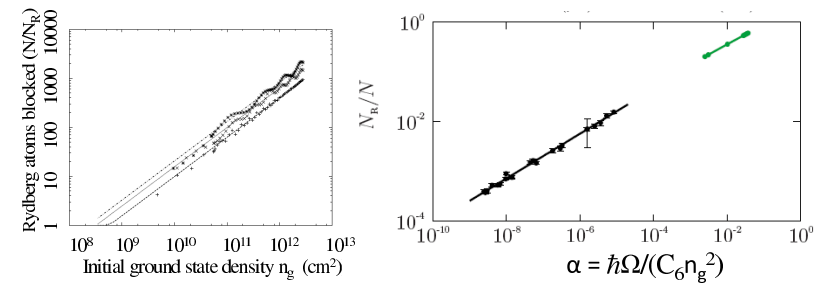

Figure 11: Scaling of the Rydberg number $N_{R}$ versus the initial atom number $N$ (or density $n_{g}$ ). Left theory (adapted from [63]), the three sets are for three different value of the (single atom) Rabi frequency $\Omega$, the line are the simple law $N_{R} \sim\left(\frac{4 \pi}{3} n_{g} \sqrt{\frac{C_{6}}{\hbar \Omega}}\right)^{4 / 5} \propto \alpha^{-0.4}$. Right (adapted from [65]). Experimental points (black) and theoretical points (green) with fit $N_{R} / N \propto \alpha^{0.45}$ for the experiment and $N_{R} / N \propto \alpha^{0.4}$ for the theory. 


\subsection{Statistic of the Rydberg excitation}

Evidence for the dipole blockade of Rydberg excitation can also examined in the statistical distributions of the number of Rydberg excitations created in ensembles of interacting Rydberg atoms. Indeed, in a simple picture, a fully saturated sample would lead to a well defined number of excited Rydberg atoms equal to the volume of the sample divided by the blockade sphere volume. The number of Rydberg atoms should then be well defined i.e. highly sub-Poissonian. This has been observed first by Georg Raithel's group (Ann Arbor, Michigan) and confirm later by the Novosibirsk's group [66, 67, 26]. Precise study of the effect on the finite detection efficiency has been performed [68, 69, 70] allowing to spectroscopically study few cold rubidium Rydberg atoms confined in a small laser excitation volume as shown in figure 12
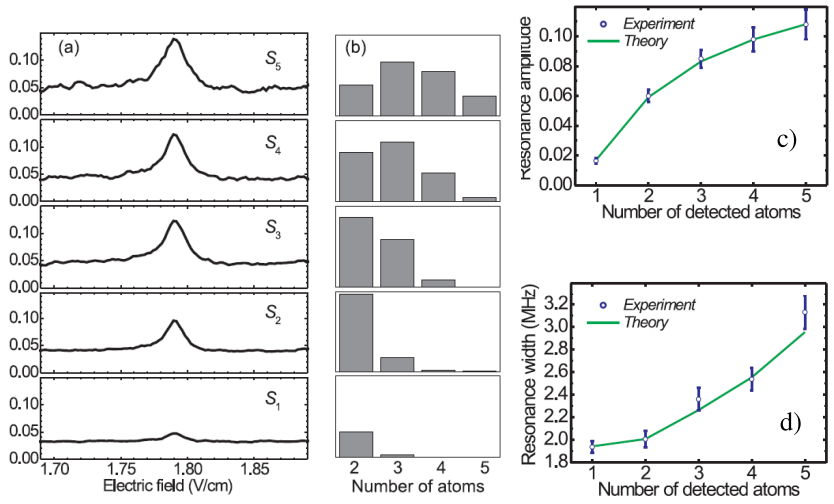

Figure 12: Small atom number Rydberg excitation. a) Experimental spectra of the Förster reaction in rubidium $37 p_{3 / 2}+37 p_{3 / 2} \rightarrow 37 s_{1 / 2}+38 s_{1 / 2}$ for atoms randomly positioned in a $(18 \mu \mathrm{m})^{3}$ cubic volume. b) Theoretical corresponding probability distributions for the number of actually interacting Rydberg atoms. $(\mathrm{c}, \mathrm{d})$ Monte Carlo Theory and experiment for the resonance amplitude and resonance width. From [70]

\subsection{Probing the band interaction: blockade or antiblock- ade effects}

Experimental investigation of the band of energy level, created by the dipoledipole interactions, has been first performed by James Martin's group (Waterloo, Canada) 71] using a radio-frequency probe transition (see figure 14). The author observe broadening of the band when adding, by RF transfer, strongly interaction Rydberg atoms. A closely related effect have been observed by T. Gallagher's group, but through direct broadening of the energy-transfer resonances $n p+n^{\prime} s \rightarrow n^{\prime} s+n p$ by introducing an additional Rydberg state $\left(n^{\prime}+1\right) p$ which does not participate directly in the energy-transfer process but is strongly 
coupled to the final states $n^{\prime} s\left(n^{\prime}=33\right.$ in the experiment) [40. Since then several group have studied similar behavior [72] (see figure 12 (d)). These experiments indicates that the measured linewidths are sensitive to the Rydberg atomic density, indicating the influence of interatomic interactions.

The difference in energy levels band spectrum in the van der Waals interaction or in a pure dipolar cases has been indeed observed using cold atoms in an optical dipole trap [73]. The experiment uses two pairs of independently tunable laser pulses to spectroscopically probe the spectrum in a double-resonance excitation scheme. By increasing the magnitude of an applied electric field, the Rydberg-atom interactions vary from van der Waals (at zero electric field) to dipole-dipole $\left(45 d_{5 / 2}+45 d_{5 / 2} \rightarrow 43 f_{7 / 2}+47 p_{3 / 2}\right)$ resonant Förster configuration, leading to characteristic signatures in the measured spectra as shown in figure 13. The zero-field spectrum (a) exhibits a wing on the negative side providing evidence for a band of two-Rydberg $(|2 r\rangle=|r r\rangle)$ excitation showing that the interactions among $45 d_{5 / 2}$ atoms are negative (attractive) and primarily van der Waals $\left(C_{6} / R^{6}\right)$ in nature. The red spectrum (b) taken at a field creating a resonant Förster configuration, exhibits symmetric wings, providing evidence for two bands $\left( \pm C_{3} / R^{3}\right)$ of $|2 r\rangle$ excitation frequencies symmetrically located consistent with the effect of a Förster resonance. It should be noted that contrary to the two atoms picture given in the bottom part of the figure no strong blockade is experimentally observed at resonance. This is due to the angular averaging effect created by all the binary interactions present (40 atoms are excited in this experiment).

This experiment highlight also that even in presence of a strong dipole interaction creates a band of energy levels, this should not necessary lead to an efficient broadening of the direct laser excitation. Indeed, the wings of the band is produced when two atoms are strongly shifted by interactions and are therefore not accessible, due to blockade, in a single excitation step, as shown by the non broadening black curve in figure 13).

Specific interatomic distance $R$ leads to specific energy position $E$ in the band structure especially in the $E=C_{6} / R^{6}$ van der Waals behavior. Therefore, by choosing the detuning of a probe laser, specific pair distance could be selected. This very attractive possibility can be observed using a very simple laser scheme. Indeed, in a two-step excitation scheme an Autler-Townes energy splitting create a detuning for the excitation step toward the Rydberg atoms as already shown in figure 4 [74. In other word pair of atoms at the distance $R=\left(C_{6} / E\right)^{1 / 6}$, where $E$ is the Autler-Townes energy shift can be specifically excited. The antiblockade has been experimentally observed recently on a random distributed MOT sample [75] and results are presented in figure [15] Data clearly show that, when the coupling energy matches the interaction energy of the Rydberg long-range interactions, the otherwise blocked excitation of close pairs becomes possible. The experiment, open the way to address specifically distance between Rydberg atoms. 


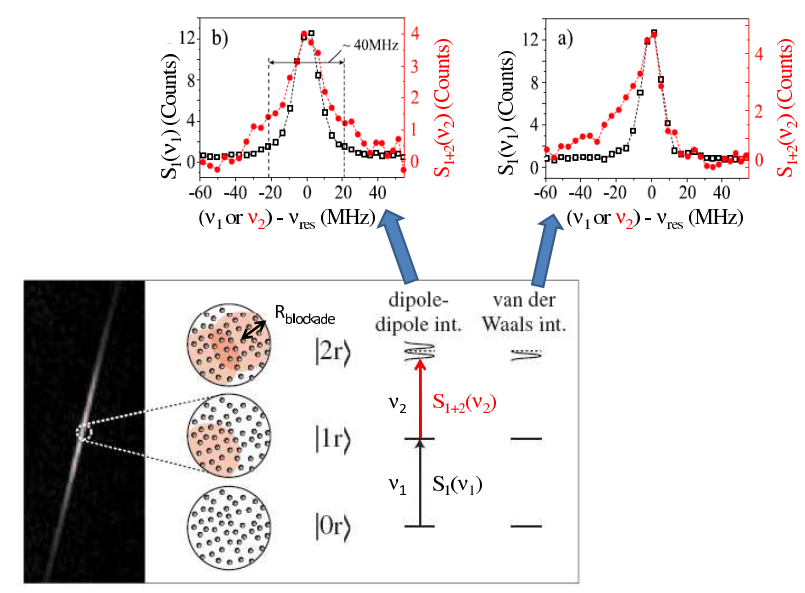

Figure 13: Experimental study of energy levels band spectrum in the van der Waals and in a pure dipolar interaction cases. The (left) low panel shows a shadow image of atoms in an optical dipole trap. The right panel shows the blockade radius $R_{b}$ as well as an excitation domain with zero, one, or two interacting Rydberg excitations. Upper figures: spectra for zero applied field (a) and for an applied field to reach the Förster resonance (b). $S_{1}\left(\nu_{1}\right)$ (black squares; left axis) scan corresponds to the transition $|0 r\rangle \rightarrow|1 r\rangle$. In order to probe the transition $|1 r\rangle \rightarrow|2 r\rangle, \nu_{1}$ is kept at resonance $\nu_{\text {res }}$ while a second pulses $\nu_{2}$ is scan and extra signal is recorded in the $S_{1+2}\left(\nu_{2}\right)$ scan (red circles; right axis). Adapted from [73].

Figure 14: Experimental investigation of the broadening of the $45 d_{5 / 2}$ Rydberg energy levels when dipole-dipole interaction (i.e. when $46 p_{3 / 2}$ atoms) is added to the system. The probing of the $45 d_{5 / 2}$ Rydberg energy levels is performed using a two-photon $45 d_{5 / 2} \rightarrow 46 d_{5 / 2}$ microwave transition. Adapted from [71].

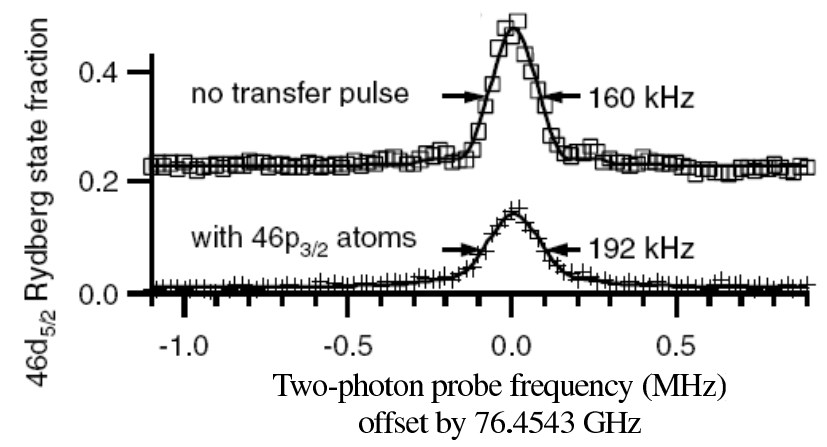


Figure 15: Observation of the antiblockade effect. Comparison between calculated (a) and measured (b) 62d Rydberg excitation spectra (upper graphs black) and Penning ionization spectra (lower graphs red) taken at different time delay. Model and experiments have an Autler-Townes splitting of $100 \mathrm{MHz}$ and a density of the trapped ground state atoms of $7 \times 10^{9} \mathrm{~cm}^{-3}$. From [75].
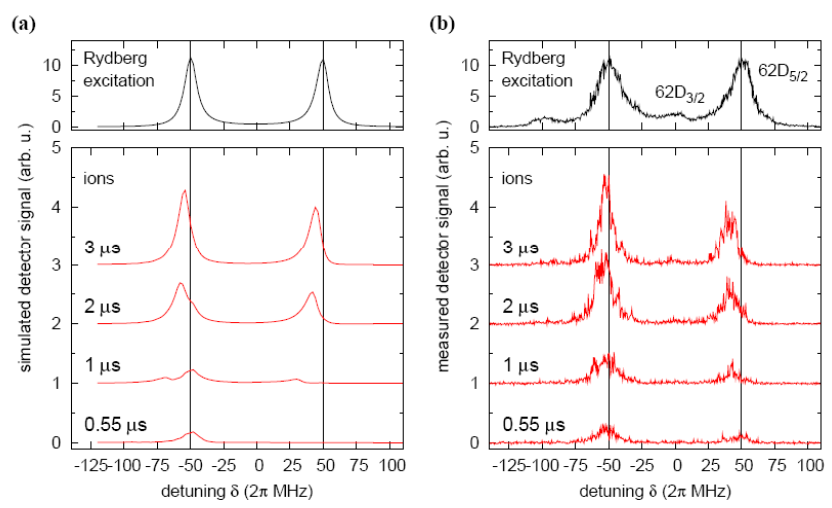

\subsection{Dipolar blockade}

\subsubsection{Experimental observation}

If the van der Waals blockade, due to $1 / R^{6}$ type of potential interaction, has been observed in 2004 , the dipole blockade itself, i.e. with $1 / R^{3}$ interaction, has been observed only in 2006 by our group; in two different experiments, FRET [76] and permanent dipole interaction [77], both using external electric field.

Figure 16: Evidence for the dipole blockade induced by electric field. (a) Excitation of the spectral lines of the $75 p_{3 / 2}$ level for different electric fields. The shift of the lines is due to the Stark effect and the reduced signal is mainly due to the dipole blockade effect. (b) Comparison of spectral lines of the $70 p_{3 / 2}$ for two different electric fields. (c) Kinetic Monte Carlo simulation. Adapted from [77.

The evidence for the dipole blockade induced by electric field is shown in Figure 16. The Rydberg atoms are excited from a cloud produced in a cesium 
vapor loaded magneto-optical trap. The laser excitation is provided by three $\mathrm{cw}$ resonant lasers simultaneously applied during a time of $300 \mathrm{~ns}$ with a repetition rate of $80 \mathrm{~Hz}$. The dipole blockade controlled through Stark effect presents an efficiency of $60 \%$ limited by the resolution of the excitation $(\sim 5 \mathrm{MHz})$. In order to avoid any discussion concerning ionic effects, the experiment has been performed at relatively low atomic density, using a short time excitation with quite low laser intensity resulting to less than one ion present per shot.

\subsubsection{Mean field and nearest neighbor approximation.}

As shown in Figure 16 c) model of the excitation including the dipole-dipole interaction of each atom with its closest Rydberg neighbor gives good agreement with the dipole blockade data. The model is the following. An atom $i$ can be excited in a Rydberg state if the excitation linewidth (taken into account the laser linewidth, laser broadening and natural lifetime) $\hbar \Delta_{\text {exc }}$ is smaller than the dipole-dipole interaction $V=\sum_{j} V_{i j}$, which is the sum of all possible interacting with neighbors. Monte Carlo simulations can be performed to choose the interatomic distances [62. Assuming rate equation for laser excitation kinetic Monte Carlo method has been chosen because it is very general, faster than usual Monte Carlo methods, and gives exacts solutions of any real system evolving through a master equation (or rate equations).

However, simplifications on the $\sum_{j} V_{i j}$ calculation can be performed to have an analytical model giving qualitative predictions.

As previously discussed, in the isotropic Van der Waals case $V_{i j} \propto \frac{1}{R_{i j}^{6}}$ and it is possible to replace the sum $\sum_{j} V_{i j}$ by an integral which leads to a mean field picture [56. This explains why the blockade sphere picture is well adapted to the isotropic van der Waals isotropic interaction. To be precise, a small angular dependence is still present in the Van der Waals interaction and the blockade sphere is indeed more a blockade ellipsoid (see Figure 13 of Reference [24]). However, blockade sphere picture and mean field approach has to be used with care for the anisotropic dipole-dipole interaction. The main reason comes from the fact that the angular average of $V_{i j}$ is zero, because $\int_{\theta=0}^{\theta=\pi}\left(1-3 \cos ^{2} \theta\right) \sin \theta d \theta=0$. More precisely, in a mean field coarse grain approach, the individual atomic position are smooth out and the sum $\sum_{j \neq i} V_{i j}$ is replace by an integral and a simple mean field approach would simply gives zero. Consequently, a mean field type of treatment has to be done carefully for instance by separating the nearest neighboring interactions in the sum. Indeed, a Monte Carlo study 62 clearly indicates that the nearest neighboring interaction plays an important, if not a dominant, role. Similarly, F. Robicheaux [78] has shown that the pair fluctuation at small separation is the dominant factor contributing to line broadening. Finally in the Förster case the pairs of very close atoms play a particular role for the observation of broad resonances. More precisely, at exact resonance the interaction has a finite spatial range when out of resonance the interaction is peaked at some distance [79].

Consequently, in a simple estimation of $\sum_{j} V_{i j}$ we can isolate the nearest 
neighbor atom from all other atoms $j$ having larger internuclear distance. For these far away atoms the values of $R_{i j}$ are regularly spaced so the angular average can be performed and leads to a mean field value, which is simply zero for atoms in the center of a spherical ensemble. For a uniform distribution of atoms, the distribution of the distance of the nearest neighbor has Erlang distribution 3 . For simplicity we could assume a single nearest-neighbor distance $R_{0}$ given by $R_{0}^{3} n_{\mathrm{Ryd}} \sim 1$. Thus $V \sim \mu(F)^{2} /\left(4 \pi \varepsilon_{0} R_{0}^{3}\right) \sim \mu(F)^{2} n_{\mathrm{Ryd}}(t) /\left(4 \pi \varepsilon_{0}\right)$ increase with time as the Rydberg density $\left(n_{\mathrm{Ryd}}\right)$. Finally the maximum achievable Rydberg density is simply $n_{\mathrm{Ryd}, \text { Max }}$ given by: $\hbar \Delta_{\mathrm{exc}} \sim \frac{\mu(F)^{2} n_{\mathrm{Ryd}, \mathrm{Max}}}{4 \pi \varepsilon_{0}} \sim n^{4} \frac{\mu_{p d}^{2} F^{2}}{\Delta_{p d}^{2} / 4} \frac{n_{\mathrm{Ryd}, \mathrm{Max}}}{4 \pi \varepsilon_{0}}$ This model is enough to qualitatively explain the results of Figure 16 and especially the fact that $n_{\mathrm{Ryd} \text {,Max }}$ decreases when $F$ increases.

If the angular average of the $1 / R^{3}$ part of the potential is zero, the $1 / R$ retarded interaction between the dipoles has an angular dependence such that the interaction does not vanish when integrated over the sample. Indeed when all dipoles are aligned we can define the angle $\theta$ by $\cos \theta=(\vec{n} \cdot \vec{\mu})$, and the retardated (generalisation of the hamiltonian given by Eq. 5) becomes:

$$
H_{12}=\frac{\mu_{1} \mu_{2}}{4 \pi \varepsilon_{0}}\left\{-\frac{k^{2} \cos (k R) \sin ^{2} \theta}{R}+\left(1-3 \cos ^{2} \theta\right)\left[\frac{\cos (k R)}{R^{3}}+\frac{k \sin (k R)}{R^{2}}\right]\right\},
$$

This formula is derived using quantum electrodynamics in the appendix in Eq. (42).

Thus, for the $1 / R$ interaction atoms which are farther apart are relatively more important than they are for the static $1 / R^{3}$ dipole-dipole interaction. An interesting feature of this interaction, which up to now has never been studied, is that it is a true collective, many atom interaction rather than many binary (nearest neighbors) interactions.

\subsection{3 $1 / R^{3}$ behavior.}

For non spherical ensemble the angular averaging of the $1 / R^{3}$ interaction is not zero and the results can be very different. This as been demonstrated by $\mathrm{H}$. B. Van Linden van den Heuvell's group (Amsterdam) 80] where the authors used, not permanent dipole interaction, but the $41 d+49 s \rightarrow 42 p+49 p$ Förster resonance. The interaction in time and space are controlled by varying the laser excitation beam separation as shown in figure 17. The result clearly show the $1 / R^{3}$ dependence by using two spatially separated atomic samples. A first volume is for the $41 d$ atoms and a second one for the $49 \mathrm{~s}$ atoms. Quantum beat oscillations in $\sin ^{2}(V t) \sim t^{2} V^{2} \propto t^{2} / R^{6}$, which are expected on the basis of the coherent coupling between atoms separated by distance $R$ are not observed because they are average (dephased) when summing over all atoms. However the spatial average $\int t^{2} /\left(x^{2}+d^{2}\right)^{3} d x$, along the $x$ coordinate of a laser, leads to a $t^{2} / d^{5}$ dependence of the signal clearly visible in figure 17

\footnotetext{
${ }^{3}$ The probability to find a $k^{t h}$ nearest neighbor at a distance $R$ is given by the Erlang distribution $4 \pi R^{2} \frac{3}{4 \pi k !} \frac{\left(R^{3}\right)^{k-1}}{\left(R_{0}^{3}\right)^{k}} e^{-\left(\frac{R}{R_{0}}\right)^{3}}$ and $R_{0}=\left(\frac{4 \pi n_{\mathrm{Ryd}}}{3}\right)^{-\frac{1}{3}}$.
} 

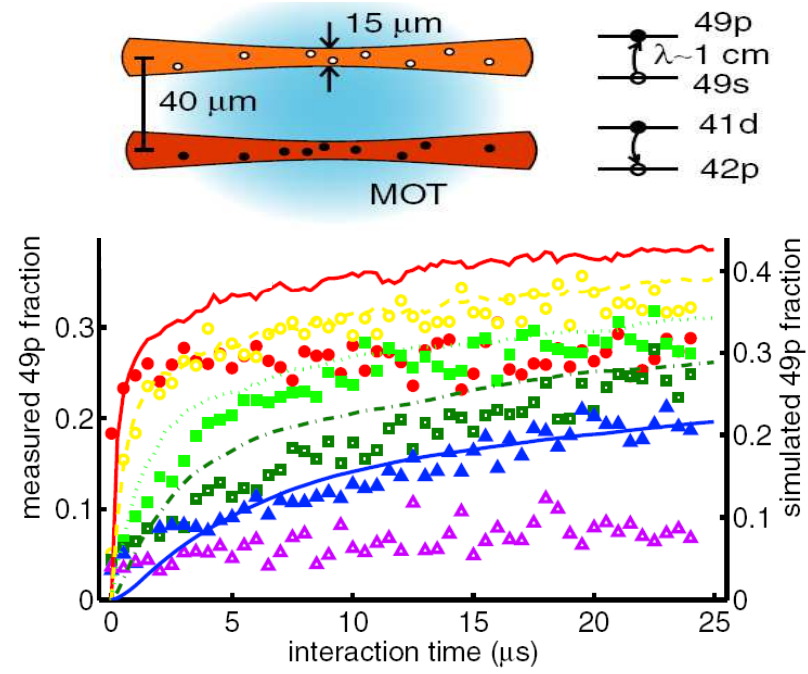

Figure 17: Spatial and time resolution of dipole-dipole interaction between Rydberg atoms. Upper part, schematic of the Förster $\left(41 d_{3 / 2}+49 s_{1 / 2} \rightarrow\right.$ $42 p_{1 / 2}+49 p_{3 / 2}$ ) experiment. Lower part: measured production of the $49 p$ state as a function of the interaction time for several separation of the cylinders: from $d=0 \mu \mathrm{m}$ (red) to $20,30,40,50 \mu \mathrm{m}$ and $\infty$ (purple). A full many-body quantum calculation, performed by F. Robicheaux, (solid line) reproduces the main features of the experiments, mainly the fact that the transfer rate scaled as $d^{-5 / 2}$ with the effective separation distance $d$. Adapted from [80].

\subsection{Blockade at Förster resonance}

The dipole blockade has also been observed at in a FRET experiment in a cesium sample (see figure 18) [76]. The efficiency of the process is characterized by the minimum of the excitation. It is limited to $30 \%$, essentially due to the relatively low $n$ considered $(n<42)$. We notice that the $n p$ excitation at the $n p+n p \rightarrow n s+(n+1) s$ Förster resonance corresponds to an efficient transfer towards the levels $n s$ an $(n+1) s$ levels. Few ions are present and are produced after the laser excitation mostly due to blackbody ionization. It is interesting to notice that the number of ions is greater for higher electric field than the field at the Förster resonance. This result is interpreted as a Penning ionization process because of the attractive force due to dipole-dipole interaction. A clear evidence of such effect will be exposed in the next section.

Complementary to this dc electric-field-induced resonant energy transfer, the ac Stark effect (Radio Frequency of micro-wave) can also be used to induce degeneracy and resonant energy transfer between cold Rydberg atoms. This has been clearly demonstrated by H. B. Van Linden van den Heuvell's team using RF fields in spatially separated volumes [81 as well as by J. D. D. Martin's group using a microwave field [82, 83]. This method for enhancing interactions 
Figure 18: Dipole blockade at Förster resonance. The Cs resonance $38 p_{3 / 2}+$ $38 p_{3 / 2} \rightarrow 38 s_{1 / 2}+39 s_{1 / 2}$ is study versus the applied electric field. Upper curve: total number of Rydberg atoms (a, black) and number of $38 \mathrm{~s}$ atoms (b, green). Lower curve: number of formed ions (c, blue). Adapted from [76].

has more flexibility due to the possibility of varying the applied frequency in addition to the amplitude as in the dc case.

\subsection{Coherence studies of Rydberg sample}

Narrowband excitation opens the way to study in more detail coherent effects with Rydberg atoms. For instance, adiabatic transfer becomes possible and have indeed been observed [84, 85]. We could also mention the work based on Coherent Population Trapping or on the Electromagnetically Induced Transparency (EIT) technique, which have been first demonstrated by C. Adams's group (Durham, UK) [86] allowing to have narrow line information [87] even in a vapor cell room temperature sample [88.

Study of coherence effect inside an ultra-cold Rydberg gas have been pioneered by T. Gallagher's group which observed a density-dependent dephasing attributed to the inhomogeneities in the exchange interactions using Ramsey's type of spectroscopy [89]. In 2005, using time-resolved narrow-band deexcitation spectroscopy, the Orsay's group studied similar coherence as well as the destruction of the Rydberg sample coherence induced by the motion of atoms due to dipolar forces [90. A clear indication of the modification of the coherence of excitation has been reported in [52]. With one atom in the trap the authors observed Rabi oscillations but, when more than one atom is in the trap they observed loss of contrast of the Rabi oscillations due to the van der Waals interaction of the excited atoms. These experiments could not distinguish between real decoherence or simple dephasing. Spin-echo, or phase reversal technique, first use by T. Pfau's team [59, 91, 92, then experimentally and theoretically study by other teams [93, 94, 95], have revealed that in a frozen gas, the evolution can indeed be hamiltonian and the coherence still present even if hidden for stan- 
dard detection methods. Finally, double crossing Landau-Zener-Stueckelberg oscillations in the dipole-dipole interaction between Rydberg atoms has been observed recently (single crossing have been studied by Pillet's group [15, 96]), in H. B. van Linden van den Heuvell's group, using an externally applied radiofrequency field, proving coherent dipole-dipole interaction during at least $0.6 \mu \mathrm{s}$ [97.

Conclusions of all these studies are that the dephasing rate is found to increase with density and that for a typical Rydberg density of $10^{9} \mathrm{at} / \mathrm{cm}^{3}$ decoherence occurs in less than $1 \mu \mathrm{s}$. This obviously put limits concerning the capability to realized quantum gate in many atoms sample [3].

Another collective or cooperative effect of cold Rydberg sample, similar to the behavior of Frenkel excitons [41, concerns their superradiant behavior as observed by many groups [98, 99]. As the theory predicts [37, 38] small atom systems show the increased emission generated by the strong dipole-dipole interaction. However, for large numbers of atoms the effect of the dipole-dipole interaction on collective emission is reduced. These non linear effects are closely related to strong coupling to light observed in micro-wave of black-body coupling in Serge Haroche's group [100, 101]. Other non linear effect such as the already mentioned EIT or four-wave mixing have been observed and are promising for studying quantum effects in blockaded atom cloud [102].

\subsection{Quantum equilibrium behavior of a Rydberg sample}

What are the equilibrium quantum properties of a dipolar gas sample? Since 2000 several theoretical studies [103, especially by G. Shlyapnikov, P. Zoller, M. Lewenstein or L. Santos' groups, have tackled this problem of dipolar gases near quantum degeneracy [104, 105]. The phase diagram of such extremely cold systems is complex and depends on the ratio of dipole-dipole interactions over contact (scattering) interactions. Such studies have obviously a lot of connection with solid state physical systems [106, 107]. Similar dipole-dipole studies can be performed using cold polar molecules [108, 109, 110, 111, 112] or magnetic dipolar gases, such as beautifully observed in strongly perturbed anisotropic expansion of a Chromium condensate by Tilman Pfau's group [113, 114]. Rydberg gases can be seen as frontier system with huge dipole-dipole interaction. "Thermodynamical" equilibrium is rich (see Figure 19) and has strong similarity with spin magnetism because the ground state of the Hamiltonian describing the system exhibits a similar phase transition in both systems [64, 112, 115]. However, the reduced lifetime and inelastic collisions have prevented, up to now, to study such equilibrium type of behavior. For instance, Tilman Pfau's group, study the Rydberg interaction inside a Bose-Einstein condensate [116] where only the high density play a role but no special quantum effect due to the coherence properties of the Condensate have been observed.

An interesting idea, which can help to study equilibrium states, is to combine the large dipole given by Rydberg states with the long lifetime given by the ground state atoms [103]. It consist to induce a the large dipole moment to long lived ground state atoms by coupling them weakly (i.e. by dressing them 
out of resonance) to Rydberg states [112. However controlling the amount of Rydberg population necessary seems challenging [117, 3].
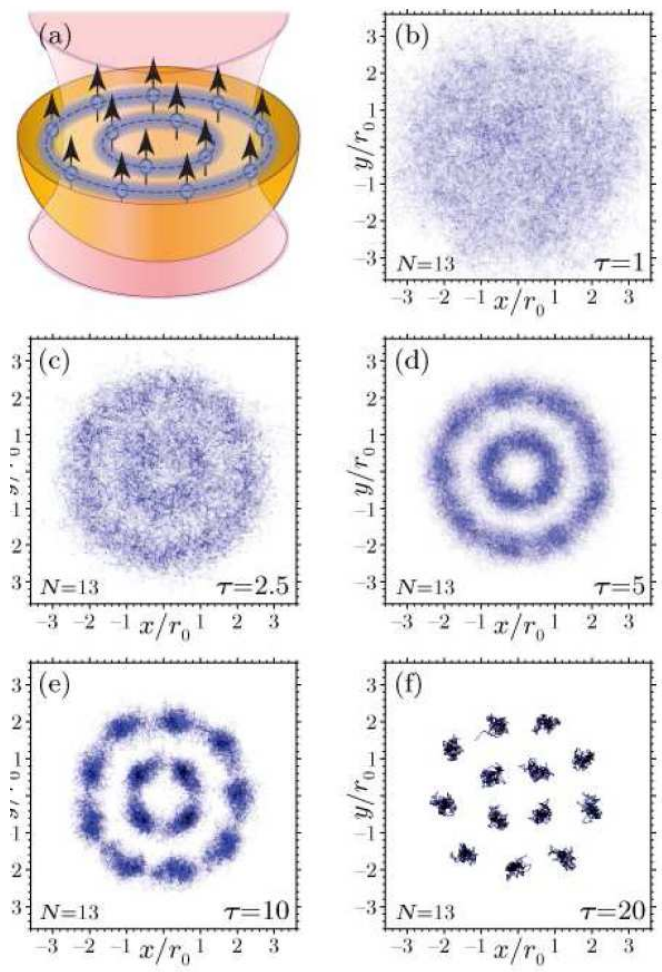

Figure 19: Ground state estimates of many Rydberg atoms system. In a possible experimental setup (scheme in a): Rydberg-dressed atoms are confined to 2D by a strong confining laser beam, with dipoles polarized perpendicular to the plane. In-plane harmonic confinement is provided e.g. by the beam waist. (b-f) Monte Carlo snapshots of the density of particles for $N=13$ dipoles, for several strength $\tau$ of the dipole-dipole interactions. (b) superfluid; (c) supersolid; (d-e) ring-like crystals; (f) classical crystal. From [112] see also [115].

\subsection{Multi-body effects in the modelization of an ultracold Rydberg gas}

Before describing difficulties presented by the many-atomic treatment, we first present what is the convenient hamiltonian description of the physics. 


\subsubsection{Laser Rydberg excitation of a large ensemble of cold atoms}

The laser excitation of several atoms is much more complex to treat that the evolution of two atoms (which is derived in detail in the appendix 9), even if we stay in a frozen gas approach. For instance in the example of the $n p+n p \leftrightarrow n s+(n+1) s$ reaction cesium atoms, the atoms can be excited in states $\left\{\left|r^{(0)}\right\rangle,\left|r^{(1)}\right\rangle,\left|r^{(2)}\right\rangle\right\}=\{|n s\rangle,|n p\rangle,|(n+1) s\rangle\}$. We also the Förster hamiltonians terms corresponding to an exchange of internal energy, $n p+n p \leftrightarrow$ $n s+(n+1) s$ and $n p+n p \leftrightarrow(n+1) s+n s$ between two atoms $i$ and $j(i$ being the first one and $j$ the second in the equations). We have also migration hamiltonian terms, corresponding to an exchange of excitation, $i(n s)+j(n p) \leftrightarrow i(n p)+j(n s)$, $(n+1) s+n p \leftrightarrow(n+1) s+n p$ and $(n+1) s+n s \leftrightarrow(n+1) s+n s$. Thus the total hamiltonian is very rich, but all reactions between an atom $i$ and an atom $j$ can be treated.

In general, if all the possible Rydberg states in the $i=1, \cdots, N$ atoms system are labeled by $r^{(n)}$, the hamiltonian (containing even off resonant terms) is given by the generalization of Eq. 7

$$
H=\sum_{i} \sum_{n} \hbar \omega_{r^{n}}\left|r_{i}^{(n)}\right\rangle\left\langle r_{i}^{(n)}\right|+\sum_{i, j} \sum_{n, n^{\prime} ; n^{\prime \prime}, n^{\prime \prime \prime}} H_{12}^{r_{i}^{(n)} r_{i}^{\left(n^{\prime}\right)} ; r_{i}^{\left(n^{\prime \prime}\right)} r_{i}^{\left(n^{\prime \prime \prime}\right)}}
$$

\subsubsection{Optical Bloch equations to study laser Rydberg excitation}

If we want to include the radiative lifetime it is necessary to describe the system using the time evolution of the density matrix $\rho$ given by the optical Bloch equations. Detail treatment has been given in [62, 50] and we are not going to enter in any detail here, but the next sections will highlight some of the difficulties. Very briefly, taking the trace over all the atoms except the one labeled $i$ in the optical Bloch equations, gives the evolution of the density matrix $\rho_{i}$ for the particle $i$. The interaction term or shift in energy for the atom $i$ due to the interaction with its neighboring atoms is $\sum_{j \neq i} \operatorname{Tr}_{j}\left[H_{i j}, \rho_{i, j}\right]$, with $H_{i j}$ the dipole-dipole interaction and $\rho_{i, j}$ the two-body density matrix for atoms $i$ and $j$. As correlations appear during the excitation, the state of the system does not remain a product state. However if the probability of excitation of a ground state atom into a Rydberg state is small, on the order of a few percent, and as long as the product of the individual density matrices is small, we can use the Hartree-Fock approximation where $\rho_{i, j} \sim \rho_{i} \otimes \rho_{j}$. Thus the interaction term can be written, schematically, as

$$
\sum_{j \neq i} \operatorname{Tr}_{j}\left[H_{i j}, \rho_{i, j}\right]=\left(\sum_{j \neq i} V_{i j} \rho_{j_{r r}}\right)\left(\rho_{i_{g r}}|g\rangle_{i i}\left\langle r\left|-\rho_{i_{e g}}\right| r\right\rangle_{i i}\langle r|\right)
$$

which is simply a shift of the Rydberg level for the atom $i$. The population in the excited state $\rho_{j_{r r}}$ is can then be replaced by a local mean value $\rho_{r r}(\vec{R})$ considered at different positions $\vec{R}$ over the whole atomic cloud. As we have seen, a naive (mean field) estimation for $\rho_{j_{r r}}$ could lead to wrong estimations and it is often better is to consider separately the nearest neighbor Rydberg atom 
from the other atoms. The shift in energy relies on the local density (gaussian distributed) $\rho_{0}(\vec{R})$ of the atoms in ground state. The shift for the atom $i$ is given by the interaction term of Eq. (9) which is finally proportional to $\rho_{r r}(\vec{r}) \rho_{0}(\vec{r})$. We can then use this approximation to solve equation the equation for an atom $i$.

\subsubsection{Full quantum mechanical treatment vs two-atom or mean field approach}

We have previously only briefly mentioned some basic results concerning the modelization of an ultracold Rydberg gas, but we would like to summarize here some of the approach or problems that theory has to face. Indeed, theoretical study of an ultracold Rydberg gas is very complex due to the numerous effects presents such as black-body radiation, quantum collective effects, dipolar forces, excitation transfer or dipole orientation effects to list few of them. All this explains why almost all experiments have tried to interpret their data using simple two-body, or one body plus mean field, picture. First, Akulin's study [14] as well as Frasier's one [118 have considered subsequent two body interactions to interpret data concerning diffusion of Rydberg excitation. The Connecticut group was the first one to proposed a many body (mean field) approach to study the Van der Waals blockade [56]. This approach has been modified to take into account the nearest neighbor dominant effect in dipole blockade case by the Orsay group [77, 62]. The same group has demonstrated, in a similar way to what has been performed by Jan Michael Rost's group (Dresden, Germany) [50], that this simple approach can be deduced from a more complex analysis of the density matrix evolution has described previously.

Francis Robicheaux studies carefully quantum effects in function of the atom number in the sample [119, 120, 63]. Similar study have been performed (for $N<10$ ) by Matthias Weidemüller's group [121]. Especially in the Förster resonance case, non perturbative many-atom Hamiltonian agree more with experimental results than sums over pairwise atomic potentials [122].

The fact that, the migration of the products of the reaction by exchange of Rydberg excitation between atoms, has to be taken into account or not is still a matter of debate [2]. Indeed, the interpretation of the line broadening mechanism has been recently reconsidered by F. Robicheaux [78] and, contradictory to the interpretation usually based on the diffusion of excitations [40, by turning off the diffusion process in the theoretical model no important change in the result was visible in the spectral linewidth.

Finally, the many body effects can also depend on the dimentionality of the sample as shown in the figure 20. At small radius, where the sample is nearly one-dimensional, many-body interactions are suppressed. But, when the sample becomes three-dimensional (large radius) many-body effects are apparent [6]. Furthermore, in a sample with larger number of atoms, the transfer occurs faster despites a lower density [39, 6].

All this, clearly point out that, contrary to the van der Waals or the static dipolar interaction, where simple binary picture are usually enough to obtain 


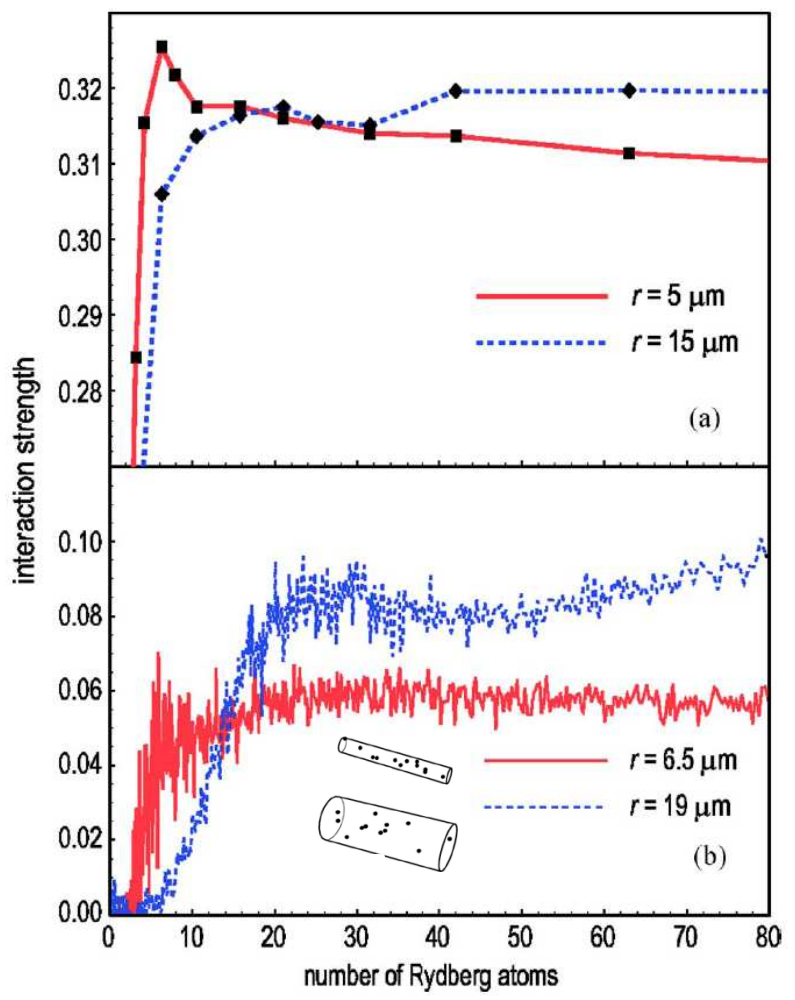

Figure 20: Evidence of many body effects and their dependence with dimensionality. a) results of the simulations of the $31 d+31 d \rightarrow 33 p+n=29$ transfer. The interaction strength (fraction of atoms that interacts) behavior reproduces qualitatively the experimental features of the data shown in part b) taken at two different excited volumes. Even though the density is higher in the smaller, one dimensional volume, the interaction is stronger in the sparser but more three-dimensional volumes. Adapted from [6].

precise results, in the Förster case it is necessary to include multiple atoms and to solve the full many-body wave function [9, 24. If the full many-body wave function is necessary to accurately model Förster experiment, the number of atoms to be included in the simulation to accurately model the experiment is still unclear (it varies from 4 to 12 [123, 122, 79]). However, only two of the states seems to play a dominant role suggesting that some simplification of the analysis may be possible by considering the atoms collectively [79]. 


\section{Control of Penning ionization}

If Penning ionizations play a key role in plasma formation they perturb the observation of blockade excitation, because the electric field created by one ion is much bigger than the one created by a Rydberg dipole. It is indeed very difficult to distinguish Rydberg blockade effects from ion plasma dynamics 61, 124, 121, 62. This has been study in our group in Orsay, by adding a simple N-body integrator (Verlet) to the Kinetic Monte Carlo simulation. We were able to describe dynamic processes in space and time and clarifies the role of collisions leading to ions in some "blockade" experiments [62].

\subsection{Evolution toward a plasma}

A cold Rydberg gas can spontaneously evolve into a plasma [16. The process is the following. Even a very slow ionization process, produces cold ions. At some point their macroscopic space charge traps all subsequently produced electrons [18, 125, 126]. For an electron temperature $T_{e}$, the number of ions $N_{\text {ion }}$ needed is simply given by the relation $\frac{N_{\text {ion }} q_{e}^{2}}{4 \pi \varepsilon_{0} l} \sim k_{B} T_{e}$ in a sample of size $l$. A trapped electron can then collide with a Rydberg atom present and ionize it leading to a free electron with a kinetic energy on the order of twice the initial Rydberg binding energy [127. This leads to a collisional avalanche which rapidly redistributes the population initially put into a single Rydberg state. At the end most of the Rydberg atoms are ionized. If Rydberg atoms can be ionized, ultracold plasma also formed Rydberg atoms in a back and forth evolution [128, 129]. Surprisingly, the Rydberg atoms in the plasma has a profound analogy with binary stars in star cluster [130]. For instance with the same Heggie's law: soft binaries get softer and hard binaries get harder. Indeed, if the Rydberg (binary) binding energy is roughly higher than a free electron (star) kinetic energy then the Rydberg atoms (binary stars) are driven to lower states.

In an initial pure Rydberg sample, the origin of the initial ions can be due to different processes such as for instance collision due to hot background atoms or ionization trough blackbody radiation. However, if exists, the dominant mechanism for this ionization is that pairs of atoms excited to attractive diatomic potential curves collide resulting in the ionization of one of the atoms, the second atom being driven to a lower state (Penning ionization process) [131, 132.

\subsection{Penning ionization between Rydberg atoms}

The key role play by the dipolar forces in the ionization process have clearly been observed in T. Gallagher's group who populated $\mathrm{Rb}(n s)$ states with a pulsed blue laser and then drive microwave transitions from the molecular nsns state to the $n s n p$ state 133 . The authors monitored the ion signal during the microwave frequency was scanned, results are shown in Figure 21. Microwave excitation to the attractive curve results in ionization, but when it is to the repulsive curve it does not. 

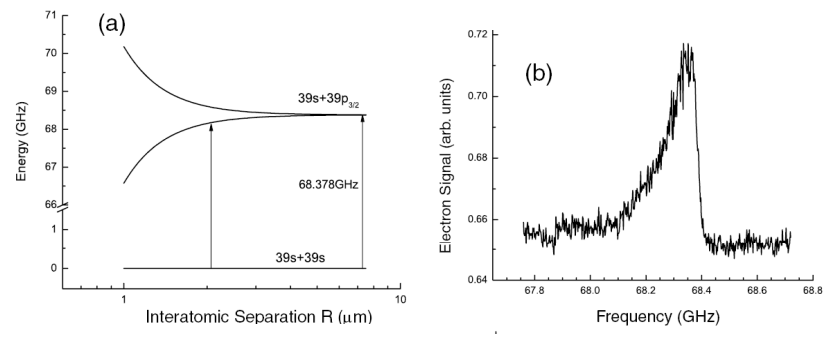

Figure 21: Penning ionization due to attractive potential curves. (a) Energy levels for the Rb pair states 39s39s and 39s39p as a function of internuclear separation (b) Plasma electron signal observed as a function of microwave frequency driving the $39 s 39 s \rightarrow 39 s 39 p$ transition; only transitions to the attractive curve of (a) are observed. From ref. [133].

If the atoms are drawn together along an attractive curve and ionize, dipoledipole potential energy is converted into kinetic energy of the resulting atom. This effect has been observed in reference 134. Assuming an $C_{n} / R^{n}$ interaction potential curve, the collisional time of two atoms (with a reduced mass equal half the individual mass $M$ ) initially separated by a distance $R_{0}$ can be very simply estimated to be $T=\int_{0}^{R_{0}} \frac{1}{\sqrt{\left(\frac{C_{n}}{R^{n}}-\frac{C_{n}}{R_{0}^{n}}\right) 4 / M}} d R$. For resonant dipole interaction $C_{3} \sim n^{2} a_{0}$ and this becomes $T \sim 20 \mu \mathrm{s} \times \frac{\sqrt{M(a m u) R_{0}^{5}(\mu m)}}{n^{2}}$. For $n=50$ rubidium atoms and an initial separation of $5 \mu \mathrm{m}$ the time is $4 \mu \mathrm{s}$ [127]. This straightforward calculation of the time required for the atoms to move along the attractive path and to ionize agrees well with experiments.

However, latter studies have raised unexpected results. Even in presence of repulsive forces, as in the van der Waals $\mathrm{Rb}(n s)$ case, Penning ionization still occur even if slower than in the case of an attractive interaction (nd states) [136. The explanation of this phenomenum is still matter of debate. Effect of Blackbody radiation transferring population towards states having attractive forces have been suggested [137, 8, 138, but the predicted rate seems too low to ensure sufficient ionization [139]. Irregularity in the arrangement of the atoms can also play a role by leading to an acceleration of the dynamics [140]. In recent experiments, a nonlinear dependence on the laser power has been observed (see figure 22) suggesting that more than one atom is excited simultaneously and that the role of dynamical Stark effect during the laser excitation must also be considered 135. Similarly, to interpret the fast $(100 \mathrm{~ns})$ ionisation of $\operatorname{Rb}(n=88)$ at a density of $5 \times 10^{10} \mathrm{~cm}^{-3}$ observed in reference [7], multiple atom absorptions has been suggested to lead to rapid ionization in a sequence of near resonant dipole-dipole transitions [7, 141].

In an effort to isolate the effect of attractive and repulsive potentials from other effects, we have examined the ionization of cold $\operatorname{Cs}(n p)$ atoms excited with narrow-bandwidth excitation [139]. The interesting features of the cesium $n p$ 


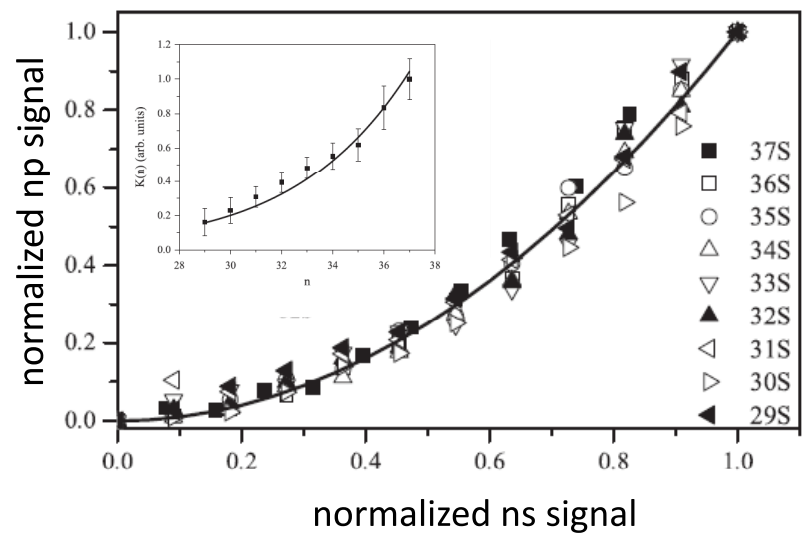

Figure 22: Observation of dynamical Stark effect during the laser excitation. Quadratic (solid line) dependence of $n p_{3 / 2}$ Rydberg signal after excitation of cold rubidium ns Rydberg atoms for several $n$ values. Because the interaction between $n s$ atoms is repulsive, the presence of $n p_{3 / 2}$ atoms are not coming from binary collision but comes from direct laser excitation facilitated by the ac Stark shift created during the laser excitation and by the dipole-dipole mixing between the $n s+n s$ and the $n p+n p$ levels. This hypothesis is attested by the inset showing the expected (for the theory see [135]) $K(n) \propto n^{8.5}$ dependence of the rate $N_{n p}=K(n) N_{n s}^{2}$. Adapted from ref. [135].

states are shown in figure 23 (a). For $n>42$ the $n p+n p$ state lies below the $n s+(n+1) s$ one, while for $n<42$ the reverse is true. The experimental ionization results, shown in figure 23 (b-c), display unambiguous difference between repulsive and attractive potentials. For $n>42$ (attractive potentials) we observe that the ion production become very non linear leading to a the complete ionization of the Rydberg sample. This is the signature of the formation of an ultracold plasma. For $n<42$ (repulsive potentials), the ionization is mainly due to blackbody radiation but we also suggest possible effect of ion-Rybderg interactions.

\subsection{Exotic molecules in Rydberg systems}

We could not conclude the review of the Rydberg blockade mechanism, i.e. on the interaction between two Rybderg atoms without mentioning that in addition to their long range part, in $C_{n} / R^{n}$, molecular potential energy surfaces reveals exotic Rydberg molecules. Indeed, the presence of many nearly degenerate pairs of states implies that the potential curves exhibit avoided crossings as a function of the interatomic distance $R$, which introduces the possibility of molecular states which can be bound. In $2000 \mathrm{C}$. Greene and H. Sadeghpour's groups predicted very peculiar levels some of them having permanent dipole moment even without any external field present. They called such states "trilobite" or 


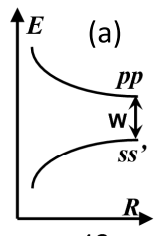

$\mathrm{n}<42$

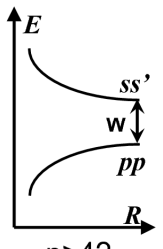

$n>42$
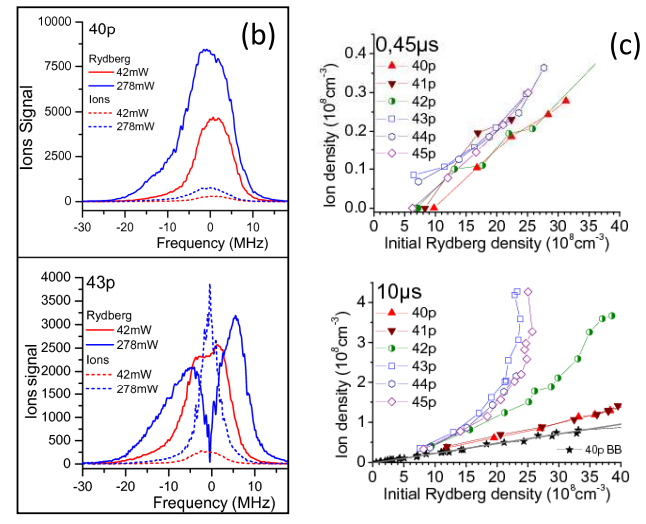

Figure 23: Penning ionization on attractive or repulsive potential curves. (a) Schematic potential curves of a pair of cesium atoms representing, as a function of internuclear distance, the energy difference (at zero electric field) between a pair of atoms in state $n p, n p(p p)$ and $n s,(n+1) s\left(s s^{\prime}\right)$. (b) Rydberg and Ion signal with two different laser intensity ( $42 \mathrm{~mW}$ and $278 \mathrm{~mW}$ ) for (upper part) $40 p$ state (repulsive) and (lower part) $43 p$ (attractive). Data are taken after $10 \mu \mathrm{s}$ of free evolution. (c) Ion density as function of initial Rydberg density for different $n$ states and two delay time (upper part) $0.45 \mu$ s and (lower part) $10 \mu \mathrm{s}$ the $40 \mathrm{p}$ BB" is taken with no $6 p$ atoms present. Adapted from ref. [142].

"butterfly" state because of the spacial electron density distribution which has analogous shape compare to trilobite or to butterfly animals [143, 144, 145]. Such molecular states involved one Rydberg electron surrounding one neutral atom. The first attempt to observe such states have been performed at Stoors (Connecticut), but only molecular potential curve crossing have been observed [146, 147]. The first observation of such states have in fact been performed in a room temperature experiment [148, 149] before being clearly observed in an ultracold ensemble [150] where spectra of the vibrational states has even been recorded as well as triatomic molecule or quantum reflection bound excited dimer states [151].

Interaction between two Rydberg atoms, has been studied in 2002 by Robin Côté's team. They predicted pure long range molecules which they call macroRydberg [152]. Similar study adding sometimes electric, magnetic or many (eventually orderered) atoms interactions have confirms the existence of many exotic Rydberg molecules [153, 154, 155, 156, 157, 74]. Poly-atomic molecules have also been predicted, even in repulsive di-atomic case because a third atoms can stabilize the system [158. Using a resolution approaching $1 \mathrm{MHz}$ Shaffer's team recently report [159] the observation of cold cesium Rydberg-atom macromolecules bound at internuclear separations of $R \sim 3-9 \mu \mathrm{m}$. The molecules are observed using the Coulomb repulsion of the ions after pulsed field ionization 
[160].

\section{Conclusion}

The Rydberg atoms with their huge electron dipole moment offer really a powerful tools for manipulating the interaction between atoms in presence of fields. Dipole-dipole interaction is the dominant feature of the cold Rydberg gas and the control of the dipole-dipole forces between Rydberg atoms opens interesting prospects [2]). The Rydberg dipole blockade offers an efficient quantum engineering for the entanglement of pairs of atoms and for the realization of quantum gates, quantum simulator or quantum repeater [?, ?]. More and more complex process can be imagined for controlling the mesoscopic system. Conditional transfer of ensemble atoms will probably be achieved in a near futur, for instance between two logical states by combining EIT and blockade [161].

The role of the dipole-dipole coupling at the Förster resonances, where multiple atoms and evolution of the full many-body wave function has to be solved, opens the way for simulating quantum many body physics [22, 23, 162]. Similarly, creation of complex many-particle states with atoms or ions in new geometries, eventually dynamical ones [163], such as lattice, make them potentially attractive for the study collective excitations [164, 165, 166], quantum random walk [167] or strongly correlated electronic system [168]. The fact that the energy levels mainly depend on the number of excited Rydberg states could be used to experimentally control, through the laser frequency, the number of excited states or even to control the interatomic distance to realize a well-defined regularly spaced Rydberg atomic sample [169, 170. The use of the strong Rydberg blockade regime, or its breakdown for specific internuclear distances, 75, 171] open the possibility to excite only nearest neighboring atoms at well defined distance [95]. This could be combined with the trapping of Rydberg atoms as realized by F. Merkt's group [1], or even coupled with laser ionization to prepare correlated plasmas [172, 173].

This review has only cover the case of alkali atoms. But very attractive fundamental as well as applicative research can be imagined using, for instance, two-valence electron atoms, such as helium, alkaline earth metals or ytterbium atoms. For instance, we might imagine using the first electron to excite the atom in a Rydberg state while manipulating the atom using the second valence electron to image, to laser cool and even to trap the Rydberg atoms.

\section{Acknowledgments}

This work is performed in the frame of the Institut Francilien pour la Recherche sur les Atomes Froids (IFRAF), of the Réseau Thématique de Recherche Avancée "Triangle de la Physique". 


\section{Appendix}

We derive here the master equation for two atoms excited in Rydberg state, taken into account retardation effect, Lamb shift effect and also (cooperative) spontaneous emission or superradiance. Other treatments can also be found in references [37, 38, 174].

\subsection{Dipole-dipole interaction between two two-level atoms}

We consider first two identical two-level $\{|1\rangle,|2\rangle\}$ atoms $A_{i} i=\{1,2\}$, interacting with the vacuum radiation $V$, in the frame of an electric dipole transition. The Hamiltonian, $H$, of the whole system $\left\{A_{i}\right\}+V$ is

$$
\begin{aligned}
H & =H_{V}+H_{A}+H_{A V} \\
H_{A} & =\sum_{i=1}^{2} H_{A i} \\
H_{A V} & =\sum_{i=1}^{2} H_{A i V}
\end{aligned}
$$

where $H_{A i}$ is the hamiltonian of the atom $i$ alone, and $H_{V}$ the hamiltonian of the vacuum radiation. We have

$$
\begin{aligned}
H_{A i} & =\hbar \omega_{1}|1\rangle_{i i}\left\langle 1\left|+\hbar \omega_{2}\right| 2\right\rangle_{i i}\langle 2| \\
H_{V} & =\sum_{l} \hbar c k \hat{a}_{l}^{+} \hat{a}_{l}
\end{aligned}
$$

$l=\left\{\vec{k}, \vec{e}_{l}\right\}$ corresponds to the modes of the vacuum radiation describes by the creation and anhilation operators $\hat{a}_{l}^{+}$and $\hat{a}_{l} . H_{A i V}$ the interaction hamiltonian coupling each atom, $A_{i}$, and the vacuum radiation $V$

$$
H_{A i V}=-\sum_{l} \varepsilon_{l}\left\langle 2\left|\vec{d} . \vec{e}_{l}\right| 1\right\rangle\left[\hat{a}_{l} \exp (i \vec{k} . \hat{\vec{R}})-\hat{a}_{l}^{+} \exp (-i \vec{k} \cdot \hat{\vec{R}})\right]|2\rangle_{i i}\langle 1|+h . c .
$$

In the frame of the rotating wave approximation, we consider only the part of the hamiltonian

$$
H_{A i V}^{R W}=-\sum_{l} \varepsilon_{l}\left\langle 2\left|\vec{d} . \vec{e}_{l}\right| 1\right\rangle \hat{a}_{l} \exp (i \vec{k} . \hat{\vec{R}})|2\rangle_{i i}\langle 1|+\text { h.c. }
$$

with $\varepsilon_{l}=i \sqrt{\frac{\hbar \omega_{l}}{2 \varepsilon_{0} L^{3}}}$. The non resonant part of the hamiltonian is

$$
H_{A i V}^{N R W}=\sum_{l} \varepsilon_{l}\left\langle 2\left|\vec{d} . \vec{e}_{l}\right| 1\right\rangle \hat{a}_{l}^{+} \exp (-i \vec{k} . \hat{\vec{R}})|2\rangle_{i i}\langle 1|+h . c .
$$




\subsection{Master equation}

The master equation is developed in the reduced density matrix approach $\sigma=$ $\operatorname{Tr}_{V}(\rho)$. The evolution of the density $\rho$ is

$$
i \hbar \frac{d \rho}{d t}=[H, \rho]
$$

which leads to write

$$
i \hbar \frac{d \sigma}{d t}=\operatorname{Tr}_{V}[H, \rho]
$$

We notice that $\operatorname{Tr}_{V}\left[H_{V}, \rho\right]=0$ and $\operatorname{Tr}_{V}\left[H_{A i}, \rho\right]=\left[H_{A i}, \sigma\right]$, which leads

$$
i \hbar \frac{d \sigma}{d t}=\left[H_{A}, \sigma\right]+\operatorname{Tr}_{R}\left[H_{A V}, \rho\right]
$$

In the frame of the interaction representation

$$
\widetilde{C}=\exp \left(i\left(H_{A}+H_{V}\right) t / \hbar\right) C \exp \left(-i\left(H_{A}+H_{V}\right) t / \hbar\right)
$$

the evolution of the density matrix $\widetilde{\rho}$ is now

$$
i \hbar \frac{d \widetilde{\rho}(t)}{d t}=\left[\widetilde{H}_{A V}(t), \widetilde{\rho}(t)\right]
$$

which can be writen

$$
i \hbar(\widetilde{\rho}(t)-\widetilde{\rho}(0))=\int_{0}^{t}\left[\widetilde{H}_{A V}\left(t^{\prime}\right), \widetilde{\rho}\left(t^{\prime}\right)\right] d t^{\prime}
$$

The evolution of the reduced density matrix $\widetilde{\sigma}$ is

$$
i \hbar \frac{d \widetilde{\sigma}(t)}{d t}=\operatorname{Tr}_{V}\left[\widetilde{H}_{A V}(t), \widetilde{\rho}(t)\right]
$$

which can also be writen as

$$
\frac{d \widetilde{\sigma}(t)}{d t}=-\frac{1}{\hbar^{2}}\left\{\operatorname{Tr}_{V}\left[\widetilde{H}_{A V}(t), \widetilde{\rho}(0)\right]+\operatorname{Tr}_{V} \int_{0}^{t}\left[\widetilde{H}_{A V}(t),\left[\widetilde{H}_{A V}\left(t^{\prime}\right), \widetilde{\rho}\left(t^{\prime}\right)\right]\right] d t^{\prime}\right\}
$$

By considering the change of variable $t^{\prime}=t-\tau$, with $\tau$ varying from 0 to $t$

$$
\frac{d \widetilde{\sigma}(t)}{d t}=-\frac{1}{\hbar^{2}}\left\{\operatorname{Tr}_{V}\left[\widetilde{H}_{A V}(t), \widetilde{\rho}(0)\right]+\operatorname{Tr}_{R} \int_{0}^{t}\left[\widetilde{H}_{A V}(t),\left[\widetilde{H}_{A V}(t-\tau), \widetilde{\rho}(t-\tau)\right]\right] d \tau\right\}
$$

\subsection{Born-Markov approximation}

We assume that at $t=0$ the electromagnetic field is the vacuum radiation $|0\rangle_{V V}\langle 0|$. More we assume the density matrix at the initial time, $\widetilde{\rho}(0)$, can be factorizable, meaning there is no correlation between the atoms and the vacuum field

$$
\widetilde{\rho}(0)=\widetilde{\sigma}(0) \otimes|0\rangle_{V V}\langle 0|
$$

To go further we make the Born-Markov approximation 
- The Born approximation consists to consider that the atoms interact always with the vacuum field an that no correlation appears between the atoms and the vacuum field,meaning

$$
\widetilde{\rho}(t)=\widetilde{\sigma}(t) \otimes|0\rangle_{V V}\langle 0|
$$

- The Markov approximation (or short memory) consists to replace $\widetilde{\sigma}(t-\tau)$ par $\tilde{\sigma}(t)$. We have

$$
\begin{aligned}
& \frac{d \widetilde{\sigma}(t)}{d t}=-\frac{1}{\hbar^{2}} \operatorname{Tr}_{V} \int_{0}^{t}\left[\widetilde{H}_{A V}(t),\left[\widetilde{H}_{A V}(t-\tau), \widetilde{\sigma}(t-\tau) \otimes|0\rangle_{V V}\langle 0|\right]\right] d \tau \\
& \frac{d \widetilde{\sigma}(t)}{d t}=-\frac{1}{\hbar^{2}} \operatorname{Tr}_{V} \int_{0}^{t}\left[\widetilde{H}_{A V}(t),\left[\widetilde{H}_{A V}(t-\tau), \widetilde{\sigma}(t) \otimes|0\rangle_{V V}\langle 0|\right]\right] d \tau
\end{aligned}
$$

\subsection{Two-atom master equation}

By considering the interaction hamiltonian in the rotating wave approximation, the master equation becomes

$$
\begin{aligned}
\frac{d \widetilde{\sigma}(t)}{d t}= & -\frac{1}{\hbar^{2}} \sum_{i, j} \sum_{l} \frac{\hbar c k}{2 \varepsilon_{0} L^{3}}\left|\left\langle 2\left|\vec{d} \cdot \vec{e}_{l}\right| 1\right\rangle\right|^{2} \\
& \int_{0}^{t}\left[|2, i\rangle\langle 1, i|\otimes| 1, j\rangle\langle 2, j| \widetilde{\sigma}(t) \exp \left(i \vec{k} .\left(\vec{R}_{i}-\vec{R}_{j}\right)\right) \exp \left(i\left(\omega_{0}-c k\right) \tau\right)+\right. \\
& +\widetilde{\sigma}(t)|2, i\rangle\langle 1, i|\otimes| 1, j\rangle\langle 2, j| \exp \left(i \vec{k} .\left(\vec{R}_{i}-\vec{R}_{j}\right)\right) \exp \left(-i\left(\omega_{0}-c k\right) \tau\right) \\
& \left.-2|1, i\rangle\langle 2, i|\widetilde{\sigma}(t)| 2, j\rangle\langle 1, j| \exp \left(-i \vec{k} .\left(\vec{R}_{i}-\vec{R}_{j}\right)\right) \cos \left(\left(\omega_{0}-c k\right) \tau\right)\right] d \tau
\end{aligned}
$$

with

$$
\sum_{l}\left|\vec{d} . \vec{e}_{l}\right|^{2} \equiv\left(\frac{L}{2 \pi}\right)^{3} \int k^{2} d k d \Omega_{\vec{k}}\left(\left|\vec{d} \cdot \vec{e}_{l_{1}}\right|^{2}+\left|\vec{d} \cdot \vec{e}_{l_{2}}\right|^{2}\right)
$$

where $\vec{e}_{l_{1}}$ et $\vec{e}_{l_{2}}$ are two orthogonal polarisations for the mode $\vec{k}:\left|\vec{d} \cdot \vec{e}_{l_{1}}\right|^{2}+$ $\left|\vec{d} \cdot \vec{e}_{l_{2}}\right|^{2}=|\vec{d}|^{2}-\left|\frac{\vec{d} . \vec{k}}{k}\right|^{2}$. The integration of the angular part $\int\left[|\vec{d}|^{2}-\left|\frac{\vec{d} \cdot \vec{k}}{k}\right|^{2}\right] d \Omega_{\vec{k}}=$ $2 \pi|\vec{d}|^{2}\left(2-\int_{0}^{\pi} \cos ^{2} \theta \sin \theta d \theta\right)=\frac{8 \pi}{3}|\vec{d}|^{2}$ gives

$$
\sum_{l}\left|\vec{d} \cdot \vec{e}_{l}\right|^{2} \equiv\left(\frac{L}{2 \pi}\right)^{3} \frac{8 \pi}{3}|\vec{d}|^{2} \int k^{2} d k
$$

By introducing the operators

$$
\begin{aligned}
& r_{i}^{+}=|2, i\rangle\langle 1, i| \\
& r_{i}^{-}=|1, i\rangle\langle 2, i|
\end{aligned}
$$


we have the master equation

$$
\begin{aligned}
\frac{d \widetilde{\sigma}(t)}{d t}= & -\frac{1}{\hbar^{2}} \frac{1}{8 \pi^{3}} \sum_{l} \sum_{i, j} \int \frac{\hbar c k}{2 \varepsilon_{0}}\left|\left\langle 2\left|\vec{d} \cdot \vec{e}_{l}\right| 1\right\rangle\right|^{2} \\
& \int_{0}^{t}\left[\left[r_{i}^{+} r_{j}^{-}, \widetilde{\sigma}(t)\right]_{+} \exp \left(i \vec{k} \cdot\left(\vec{R}_{i}-\vec{R}_{j}\right)\right) \cos \left(\left(\omega_{0}-c k\right) \tau\right)\right. \\
& -2 r_{j}^{-} \widetilde{\sigma}(t) r_{i}^{+} \exp \left(i \vec{k} \cdot\left(\vec{R}_{i}-\vec{R}_{j}\right)\right) \cos \left(\left(\omega_{0}-c k\right) \tau\right) \\
& \left.+i\left[r_{i}^{+} r_{j}^{-}, \widetilde{\sigma}(t)\right] \exp \left(i \vec{k} \cdot\left(\vec{R}_{i}-\vec{R}_{j}\right)\right) \sin \left(\left(\omega_{0}-c k\right) \tau\right)\right] d \tau d^{3} \vec{k}
\end{aligned}
$$

The terms, non considered in the above equation, corresponding to the non resonant part of the hamiltonian are

$$
\begin{aligned}
\frac{d \widetilde{\sigma}^{(N R 1)}(t)}{d t}= & -\frac{1}{\hbar^{2}} \frac{1}{8 \pi^{3}} \sum_{l} \sum_{i, j} \int \frac{\hbar c k}{2 \varepsilon_{0}}\left|\left\langle 2\left|\vec{d} \cdot \vec{e}_{l}\right| 1\right\rangle\right|^{2} \\
& \int_{0}^{t}\left[\left[r_{i}^{-} r_{j}^{+}, \widetilde{\sigma}(t)\right]_{+} \exp \left(i \vec{k} \cdot\left(\vec{R}_{i}-\vec{R}_{j}\right)\right) \cos \left(\left(-\omega_{0}-c k\right) \tau\right)\right. \\
& -2 r_{j}^{+} \widetilde{\sigma}(t) r_{i}^{-} \exp \left(i \vec{k} \cdot\left(\vec{R}_{i}-\vec{R}_{j}\right)\right) \cos \left(\left(-\omega_{0}-c k\right) \tau\right) \\
& \left.+i\left[r_{i}^{-} r_{j}^{+}, \widetilde{\sigma}(t)\right] \exp \left(i \vec{k} \cdot\left(\vec{R}_{i}-\vec{R}_{j}\right)\right) \sin \left(\left(-\omega_{0}-c k\right) \tau\right)\right] d \tau d^{3} \vec{k}
\end{aligned}
$$

There is also crossed terms of the resonant and non resonant parts of the hamiltonian containing $\exp \left( \pm 2 i \omega_{0} t\right)$ phase evolution and that we are going to neglect because of their off-resonant nature. The conditions of validity for this approximation correspond to a slow variation of the density matrix, $\sigma$, compared to the period, $2 \pi / \omega_{0}$.

We choose the axis, $z$, as $\vec{R}_{i}-\vec{R}_{j},\left(\vec{e}_{z}=\left(\vec{R}_{i}-\vec{R}_{j}\right) /\left|\vec{R}_{i}-\vec{R}_{j}\right|\right)$. The emission mode, $\vec{k}$, is characterized by the angles $\theta^{\prime}$ and $\varphi^{\prime}$, and the polarisations $\vec{e}_{l 1}=\sin \varphi^{\prime} \vec{e}_{x}-\cos \varphi^{\prime} \vec{e}_{y}$ and $\vec{e}_{l 2}=-\cos \theta^{\prime} \cos \varphi^{\prime} \vec{e}_{x}-\cos \theta^{\prime} \sin \varphi^{\prime} \vec{e}_{y}+\sin \theta^{\prime} \vec{e}_{z}$. The spherical coordonates of the transition dipole vector $\vec{d}$ are $\left\{d, \theta_{i j}=\theta, \varphi_{i j}=\varphi\right\}$. We have

$$
\begin{gathered}
\vec{k} \cdot\left(\vec{R}_{i}-\vec{R}_{j}\right)=k R_{i j} \cos \theta^{\prime} \\
\sum_{l}\left|\left\langle 2\left|\vec{d} \cdot \vec{e}_{l}\right| 1\right\rangle\right|^{2}=|\langle 2|\vec{d}| 1\rangle|^{2}-\left|\left\langle 2\left|\frac{\vec{d} \cdot \vec{k}}{k}\right| 1\right\rangle\right|^{2} \\
\left|\left\langle 2\left|\frac{\vec{d} \cdot \vec{k}}{k}\right| 1\right\rangle\right|^{2}=|\langle 2|\vec{d}| 1\rangle|^{2}\left(\cos \theta \cos \theta^{\prime}+\sin \theta \sin \theta^{\prime} \cos \left(\varphi-\varphi^{\prime}\right)\right)^{2}
\end{gathered}
$$

For large $t$, we can write

$$
\int_{0}^{t \approx \infty}\left[\exp i\left(c k-\omega_{0}\right) \tau\right] d \tau=\frac{\pi}{c} \delta\left(k-\frac{\omega_{0}}{c}\right)+\frac{i}{c} P P \frac{1}{k-\frac{\omega_{0}}{c}}
$$




$$
\begin{aligned}
\frac{d \widetilde{\sigma}(t)}{d t}= & -\frac{1}{\hbar^{2}} \frac{1}{8 \pi^{3}} \sum_{i, j} \int \frac{\hbar c k}{2 \varepsilon_{0}}|\langle 2|\vec{d}| 1\rangle|^{2} \\
& \left(1-\left(\cos \theta \cos \theta^{\prime}+\sin \theta \sin \theta^{\prime} \cos \left(\varphi-\varphi^{\prime}\right)\right)^{2}\right) \\
& {\left[\left[\left[r_{i}^{+} r_{j}^{-}, \widetilde{\sigma}(t)\right]_{+}-2 r_{j}^{-} \widetilde{\sigma}(t) r_{i}^{+}\right] \exp \left(i k R_{i j} \cos \theta^{\prime}\right) \frac{\pi}{c} \delta\left(k-k_{0}\right)\right.} \\
+ & \left.i\left[r_{i}^{+} r_{j}^{-}, \widetilde{\sigma}(t)\right] \exp \left(i k R_{i j} \cos \theta^{\prime}\right) \frac{1}{c} P P \frac{1}{k-k_{0}}\right] k^{2} d k \sin \theta^{\prime} d \theta^{\prime} d \varphi^{\prime} \\
\frac{d \widetilde{\sigma}(t)}{d t}= & -\frac{1}{\hbar^{2}} \frac{1}{8 \pi^{3}} \sum_{i, j} \int_{k=0}^{+\infty} \int_{u=-1}^{+1} \frac{\hbar c k}{2 \varepsilon_{0}}|\langle 2|\vec{d}| 1\rangle|^{2} \pi \\
& \left(1+\cos ^{2} \theta+\left(1-3 \cos ^{2} \theta\right) u^{2}\right) \\
& {\left[\left[\left[r_{i}^{+} r_{j}^{-}, \widetilde{\sigma}(t)\right]_{+}-2 r_{j}^{-} \widetilde{\sigma}(t) r_{i}^{+}\right] \exp \left(i k R_{i j} u\right) \frac{\pi}{c} \delta\left(k-k_{0}\right)\right.} \\
& \left.+i\left[r_{i}^{+} r_{j}^{-}, \widetilde{\sigma}(t)\right] \exp \left(i k R_{i j} u\right) \frac{1}{c} P P \frac{1}{k-k_{0}}\right] k^{2} d k d u
\end{aligned}
$$

We should add the contribution of the non-resonant terms

$$
\begin{aligned}
\frac{d \widetilde{\sigma}^{(N R 1)}(t)}{d t}= & -\frac{1}{\hbar^{2}} \frac{1}{8 \pi^{3}} \sum_{i, j} \int_{k=0}^{+\infty} \int_{u=-1}^{+1} \frac{\hbar c k}{2 \varepsilon_{0}}|\langle 2|\vec{d}| 1\rangle|^{2} \pi \\
& \left(1+\cos ^{2} \theta+\left(1-3 \cos ^{2} \theta\right) u^{2}\right) \\
& {\left[\left[r_{i}^{-} r_{j}^{+}, \widetilde{\sigma}(t)\right]_{+} \exp \left(i k R_{i j} u\right) \frac{\pi}{c} \delta\left(k+k_{0}\right)\right.} \\
& \left.+i\left[r_{i}^{-} r_{j}^{+}, \widetilde{\sigma}(t)\right] \exp \left(i k R_{i j} u\right) \frac{1}{c} P P \frac{1}{k+k_{0}}\right] k^{2} d k d u
\end{aligned}
$$

By changing $k$ in $-k$ in the integral, we have

$$
\begin{aligned}
\frac{d \widetilde{\sigma}^{(N R 1)}(t)}{d t}= & -\frac{1}{\hbar^{2}} \frac{1}{8 \pi^{3}} \sum_{i, j} \int_{k=-\infty}^{0} \int_{u=-1}^{+1} \frac{\hbar c k}{2 \varepsilon_{0}}|\langle 2|\vec{d}| 1\rangle|^{2} \pi \\
& \left(1+\cos ^{2} \theta+\left(1-3 \cos ^{2} \theta\right) u^{2}\right) \\
& {\left[\left[r_{i}^{-} r_{j}^{+}, \widetilde{\sigma}(t)\right]_{+} \exp \left(-i k R_{i j} u\right) \frac{\pi}{c} \delta\left(-k+k_{0}\right)\right.} \\
& \left.+i\left[r_{i}^{-} r_{j}^{+}, \widetilde{\sigma}(t)\right] \exp \left(-i k R_{i j} u\right) \frac{1}{c} P P \frac{1}{k-k_{0}}\right] k^{2} d k d u
\end{aligned}
$$

The operators $r_{i}^{ \pm}$and $r_{j}^{ \pm}$commute when $i \neq j$. By inverting the indices $i$ and 
$j$, we have to replace $u$ by $-u$

$$
\begin{aligned}
\frac{d \widetilde{\sigma}^{(N R 1)}(t)}{d t}= & -\frac{1}{\hbar^{2}} \frac{1}{8 \pi^{3}} \sum_{i, j} \int_{k=-\infty}^{0} \int_{u=-1}^{+1} \frac{\hbar c k}{2 \varepsilon_{0}}|\langle 2|\vec{d}| 1\rangle|^{2} \pi \\
& \left(1+\cos ^{2} \theta+\left(1-3 \cos ^{2} \theta\right) u^{2}\right) \\
& {\left[\left[r_{i}^{+} r_{j}^{-}, \widetilde{\sigma}(t)\right]_{+} \exp \left(i k R_{i j} u\right) \frac{\pi}{c} \delta\left(k-k_{0}\right)\right.} \\
& \left.+i\left[r_{i}^{+} r_{j}^{-}, \widetilde{\sigma}(t)\right] \exp \left(i k R_{i j} u\right) \frac{1}{c} P P \frac{1}{k-k_{0}}\right] k^{2} d k d u
\end{aligned}
$$

By taking into account both resonant and non-resonant terms, we have

$$
\begin{aligned}
\frac{d \widetilde{\sigma}(t)}{d t}= & -\Gamma \sum_{i}\left[\left[r_{i}^{+} r_{i}^{-}, \widetilde{\sigma}(t)\right]_{+}-2 r_{i}^{-} \widetilde{\sigma}(t) r_{i}^{+}\right] \\
& -\frac{1}{\hbar^{2}} \frac{1}{8 \pi^{3}} \sum_{i \neq, j} \int_{k=-\infty}^{+\infty} \int_{u=-1}^{+1} \frac{\hbar c k}{2 \varepsilon_{0}}|\langle 2|\vec{d}| 1\rangle|^{2} \pi \\
& \left(1+\cos ^{2} \theta+\left(1-3 \cos ^{2} \theta\right) u^{2}\right) \\
& {\left[\left[r_{i}^{+} r_{j}^{-}, \widetilde{\sigma}(t)\right]_{+} \exp \left(i k R_{i j} u\right) \frac{\pi}{c} \delta\left(k-k_{0}\right)\right.} \\
& \left.+i\left[r_{i}^{+} r_{j}^{-}, \widetilde{\sigma}(t)\right] \exp \left(i k R_{i j} u\right) \frac{1}{c} P P \frac{1}{k-k_{0}}\right] k^{2} d k d u
\end{aligned}
$$

We do not consider the term of the principal part $P P$ in the integral for $i=j$. This term is already taken into account in the energy of the different levels in the Lamb shift calculation. We use the equation

$$
\int_{-\infty}^{+\infty} \exp \left(i\left(k-k_{0}\right) R_{i j} u\right)\left[\pi \delta\left(k-k_{0}\right)+i P P \frac{1}{k-k_{0}}\right] d k=2 \pi \Theta\left(R_{i j} u\right)
$$

with $\Theta(x)=1$ is the Heaviside function with for $x>0, \Theta(x)=1$, for $x=0, \Theta(x)=1 / 2$, for $x<0, \Theta(x)=0$. For integrating, we notice also that 
$k^{3} \exp \left(i k R_{i j} u\right)=\frac{i}{R_{i j}^{3}} \frac{\partial^{3}}{\partial u^{3}} \exp \left(i k R_{i j} u\right)$. We can write

$$
\begin{aligned}
\frac{d \widetilde{\sigma}(t)}{d t}= & -\Gamma \sum_{i}\left[\left[r_{i}^{+} r_{i}^{-}, \tilde{\sigma}(t)\right]_{+}-2 r_{i}^{-} \tilde{\sigma}(t) r_{i}^{+}\right] \\
& -\frac{1}{\hbar} \frac{1}{4 \pi \varepsilon_{0}} \sum_{i \neq j}|\langle 2|\vec{d}| 1\rangle|^{2} \\
& \left\{( 1 - \operatorname { c o s } ^ { 2 } \theta ) \frac { ( - i ) k _ { 0 } ^ { 2 } } { R _ { i j } } \left[r_{i}^{+} r_{j}^{-} \widetilde{\sigma}(t) \exp \left(i k_{0} R_{i j}\right)\right.\right. \\
& \left.-\widetilde{\sigma}(t) r_{i}^{+} r_{j}^{-} \exp \left(-i k_{0} R_{i j}\right)\right] \\
& -\left(1-3 \cos ^{2} \theta\right) \frac{-k_{0}}{R_{i j}^{2}}\left[r_{i}^{+} r_{j}^{-} \widetilde{\sigma}(t) \exp \left(i k_{0} R_{i j}\right)\right. \\
& \left.+\widetilde{\sigma}(t) r_{i}^{+} r_{j}^{-} \exp \left(-i k_{0} R_{i j}\right)\right] \\
& +\left(1-3 \cos ^{2} \theta\right) \frac{i}{R_{i j}^{3}}\left[r_{i}^{+} r_{j}^{-} \tilde{\sigma}(t) \exp \left(i k_{0} R_{i j}\right)\right. \\
& \left.\left.-\widetilde{\sigma}(t) r_{i}^{+} r_{j}^{-} \exp \left(-i k_{0} R_{i j}\right)\right]\right\}
\end{aligned}
$$

Finally, the equation can be written

$$
\begin{aligned}
\frac{d \widetilde{\sigma}(t)}{d t}= & -\Gamma \sum_{i}\left[\left[r_{i}^{+} r_{i}^{-}, \widetilde{\sigma}(t)\right]_{+}-2 r_{i}^{-} \widetilde{\sigma}(t) r_{i}^{+}\right] \\
& -\frac{1}{\hbar} \frac{1}{4 \pi \varepsilon_{0}} \sum_{i \neq j}|\langle 2|\vec{d}| 1\rangle|^{2} \\
& \left\{\left\{\left(1-\cos ^{2} \theta\right) \frac{k_{0}^{2}}{R_{i j}} \sin \left(k_{0} R_{i j}\right)\right.\right. \\
& +\left(1-3 \cos ^{2} \theta\right) \frac{k_{0}}{R_{i j}^{2}} \cos \left(k_{0} R_{i j}\right) \\
& \left.-\left(1-3 \cos ^{2} \theta\right) \frac{1}{R_{i j}^{3}} \sin \left(k_{0} R_{i j}\right)\right\} \\
& {\left[r_{i}^{+} r_{j}^{-}, \widetilde{\sigma}(t)\right]+} \\
& -i\left\{\left(1-\cos ^{2} \theta\right) \frac{k_{0}^{2}}{R_{i j}} \cos \left(k_{0} R_{i j}\right)\right. \\
& -\left(1-3 \cos ^{2} \theta\right) \frac{k_{0}}{R_{i j}^{2}} \sin \left(k_{0} R_{i j}\right) \\
& \left.-\left(1-3 \cos ^{2} \theta\right) \frac{1}{R_{i j}^{3}} \cos \left(k_{0} R_{i j}\right)\right\} \\
& {\left.\left[r_{i}^{+} r_{j}^{-}, \widetilde{\sigma}(t)\right]\right\} }
\end{aligned}
$$

\subsection{Dipole-dipole interaction}


The master equation can be written

$i \hbar \frac{d \widetilde{\sigma}(t)}{d t}=-\Gamma \sum_{i}\left[\left[r_{i}^{+} r_{i}^{-}, \widetilde{\sigma}(t)\right]_{+}-2 r_{i}^{-} \widetilde{\sigma}(t) r_{i}^{+}\right]+\sum_{i \neq j}\left[\widetilde{H}_{i j}, \widetilde{\sigma}(t)\right]-\frac{i}{2}\left(\widetilde{\Gamma}_{i j} \widetilde{\sigma}(t)+\widetilde{\sigma}(t) \widetilde{\Gamma}_{i j}\right)$

with

$$
\begin{aligned}
\widetilde{H}_{i j}= & \frac{1}{4 \pi \varepsilon_{0}}|\langle 2|\vec{d}| 1\rangle|^{2} r_{i}^{+} r_{j}^{-} \\
& \left\{-\left(1-\cos ^{2} \theta\right) \frac{k_{0}^{2}}{R_{i j}} \cos \left(k_{0} R_{i j}\right)\right. \\
& +\left(1-3 \cos ^{2} \theta\right) \frac{k_{0}}{R_{i j}^{2}} \sin \left(k_{0} R_{i j}\right) \\
& \left.+\left(1-3 \cos ^{2} \theta\right) \frac{1}{R_{i j}^{3}} \cos \left(k_{0} R_{i j}\right)\right\}
\end{aligned}
$$

and

$$
\begin{aligned}
\frac{\widetilde{\Gamma}_{i j}}{2}=\quad & -i \frac{1}{4 \pi \varepsilon_{0}}|\langle 2|\vec{d}| 1\rangle|^{2} r_{i}^{+} r_{j}^{-} \\
& \left\{\left(1-\cos ^{2} \theta\right) \frac{k_{0}^{2}}{R_{i j}} \sin \left(k_{0} R_{i j}\right)\right. \\
& +\left(1-3 \cos ^{2} \theta\right) \frac{k_{0}}{R_{i j}^{2}} \cos \left(k_{0} R_{i j}\right) \\
& \left.-\left(1-3 \cos ^{2} \theta\right) \frac{1}{R_{i j}^{3}} \sin \left(k_{0} R_{i j}\right)\right\}
\end{aligned}
$$

$\widetilde{H}_{i j}$ can be interpreted in terms of interaction between two oscillating electric dipoles, $\vec{\mu}_{i}$ and $\vec{\mu}_{j}$, at a distance $R_{i j} \vec{n}_{i j}$. This dipole-dipole interaction between two atoms can be interpreted as a collective Lamb shift due to the dipoledipole interaction. The term in $R^{-3}$ correspond to the classical dipole-dipole interactionThe term in $1 / R$ correspond to the delayed terms of the interaction.

The $\widetilde{\Gamma}_{i j}$ terms contains the spontaneous emission by individual atoms.

\section{References}

[1] S. D. Hogan and F. Merkt. Demonstration of three-dimensional electrostatic trapping of state-selected rydberg atoms. Physical Review Letters, 100(4):043001-+, February 2008.

[2] Th.F. Gallagher and P. Pillet. Dipole - dipole interactions of rydberg atoms. Advances in Atomic, Molecular, and Optical Physics, 56:161, 2008.

[3] M. Saffman, T. G. Walker, and K. Molmer. Quantum information with rydberg atoms. ArXiv e-prints, September 2009. 
[4] M. D. Lukin, M. Fleischhauer, R. Cote, L. M. Duan, D. Jaksch, J. I. Cirac, and P. Zoller. Dipole blockade and quantum information processing in mesoscopic atomic ensembles. Physical Review Letters, 87(3):037901-+, July 2001.

[5] Thomas F. Gallagher. Rydberg Atoms. Cambridge University Press, Cambridge, 1994.

[6] T. J. Carroll, S. Sunder, and M. W. Noel. Many-body interactions in a sample of ultracold rydberg atoms with varying dimensions and densities. Phys. Rev. A, 73(3):032725-+, March 2006.

[7] P. J. Tanner, J. Han, E. S. Shuman, and T. F. Gallagher. Many-body ionization in a frozen rydberg gas. Physical Review Letters, 100(4):043002+, February 2008.

[8] A. Reinhard, T. Cubel Liebisch, K. C. Younge, P. R. Berman, and G. Raithel. Rydberg-rydberg collisions: Resonant enhancement of state mixing and penning ionization. Physical Review Letters, 100(12):123007+ , March 2008.

[9] T. G. Walker and M. Saffman. Zeros of rydberg rydberg föster interactions. Journal of Physics B Atomic Molecular Physics, 38:309-+, January 2005.

[10] K. A. Safinya, J. F. Delpech, F. Gounand, W. Sandner, and T. F. Gallagher. Resonant rydberg-atom-rydberg-atom collisions. Physical Review Letters, 47:405-408, August 1981.

[11] T. F. Gallagher. Resonant collisional energy transfer between rydberg atoms. Phys. Rep., 210:319-366, January 1992.

[12] W. R. Anderson, J. R. Veale, and T. F. Gallagher. Resonant dipole-dipole energy transfer in a nearly frozen rydberg gas. Phys. Rev. Lett., 80:249, 1998.

[13] I. Mourachko, D. Comparat, F. de Tomasi, A. Fioretti, P. Nosbaum, V. M. Akulin, and P. Pillet. Many-body effects in a frozen rydberg gas. Phys. Rev. Lett., 80:253, 1998.

[14] V. M. Akulin, F. D. Tomasi, I. Mourachko, and P. Pillet. Level-band problem and many-body effects in cold rydberg atoms. Physica D Nonlinear Phenomena, 131:125-140, July 1999.

[15] A. Fioretti, D. Comparat, C. Drag, T.F. Gallagher, and P. Pillet. Longrange forces between cold atoms. Phys. Rev. Lett., 82:1839, 1999.

[16] G. Vitrant, J. M. Raimond, M. Gross, and S. Haroche. Rydberg to plasma evolution in a dense gas of very excited atoms. J. Phys. B, 15:L49, 1982. 
[17] T. C. Killian, S. Kulin, S. D. Bergeson, L. A. Orozco, C. Orzel, and S. L. Rolston. Creation of an ultracold neutral plasma. Phys. Rev. Lett., 83(23):4776, 1999.

[18] M P Robinson, B L Tolra, M W Noel, T F Gallagher, and P Pillet. Spontaneous evolution of rydberg atoms into an ultracold plasma. Phys. Rev. Lett., 85:4466, 2000.

[19] D. Jaksch, J. I. Cirac, P. Zoller, S. L. Rolston, R. Côté, and M. D. Lukin. Fast quantum gates for neutral atoms. Physical Review Letters, 85:22082211, September 2000.

[20] A. Gaëtan, Y. Miroshnychenko, T. Wilk, A. Chotia, M. Viteau, D. Comparat, P. Pillet, A. Browaeys, and P. Grangier. Observation of collective excitation of two individual atoms in the rydberg blockade regime. Nature Physics, 5:115-118, February 2009.

[21] L. Isenhower, E. Urban, X. L. Zhang, A. T. Gill, T. Henage, T. A. Johnson, T. G. Walker, and M. Saffman. Demonstration of a Neutral Atom Controlled-NOT Quantum Gate. Physical Review Letters, 104(1):010503+ , January 2010.

[22] M. Lewenstein, A. Sanpera, V. Ahufinger, B. Damski, A. Sen, and U. Sen. Ultracold atomic gases in optical lattices: mimicking condensed matter physics and beyond. Advances in Physics, 56:243-379, March 2007.

[23] I. Buluta and F. Nori. Quantum simulators. Science, 326:108-, October 2009.

[24] T. G. Walker and M. Saffman. Consequences of zeeman degeneracy for the van der waals blockade between rydberg atoms. Phys. Rev. A, 77(3):032723-+, March 2008.

[25] M. W. Horbatsch, M. Horbatsch, and E. A. Hessels. A universal formula for the accurate calculation of hydrogenic lifetimes. Journal of Physics B Atomic Molecular Physics, 38:1765-1771, June 2005.

[26] I. I. Beterov, I. I. Ryabtsev, D. B. Tretyakov, and V. M. Entin. Quasiclassical calculations of blackbody-radiation-induced depopulation rates and effective lifetimes of rydberg ns, np, and nd alkali-metal atoms with n $<80$. Phys. Rev. A, 79(5):052504-+, May 2009.

[27] J. W. Farley and W. H. Wing. Accurate calculation of dynamic stark shifts and depopulation rates of rydberg energy levels induced by blackbody radiation. hydrogen, helium, and alkali-metal atoms. Phys. Rev. A, 23:2397-2424, May 1981.

[28] B. Kaulakys. Consistent analytical approach for the quasi-classical radial dipole matrix elements. Journal of Physics B Atomic Molecular Physics, 28:4963-4971, December 1995. 
[29] B. Oumarou, J. Picart, N. Tran Minh, and J. Chapelle. New and rapid method for calculation of electric dipole and quadrupole radial integrals between atomic rydberg states. Phys. Rev. A, 37:1885-1894, March 1988.

[30] G. Lagmago Kamta, S. G. Nana Engo, M. G. K. Njock, and B. Oumarou. Consistent description of klein-gordon dipole matrix elements. Journal of Physics B Atomic Molecular Physics, 31:963-997, March 1998.

[31] L. G. D'yachkov and P. M. Pankratov. On the use of the semiclassical approximation for the calculation of oscillator strengths and photoionization cross sections. Journal of Physics B Atomic Molecular Physics, 27:461-472, February 1994.

[32] J. H. Hoogenraad and L. D. Noordam. Rydberg atoms in far-infrared radiation fields. I. Dipole matrix elements of $\mathrm{H}, \mathrm{Li}$ and Rb. Phys. Rev. A, 57(6):4533, june 1998.

[33] Myron L. Zimmerman, Michael G. Littman, Michael M. Kash, and Daniel Kleppner. Stark structure of the rydberg states of alkali-metal atoms. Phys. Rev. A, 20(6):2251-2275, Dec 1979.

[34] K. Singer, J. Stanojevic, M. Weidemüller, and R. Côté. Long-range interactions between alkali rydberg atom pairs correlated to the ns ns, np np and nd nd asymptotes. Journal of Physics B Atomic Molecular Physics, $38: 295-+$, January 2005.

[35] A. Reinhard, T. C. Liebisch, B. Knuffman, and G. Raithel. Level shifts of rubidium rydberg states due to binary interactions. Phys. Rev. A, 75(3):032712-+, March 2007.

[36] A. Reinhard, T. C. Liebisch, B. Knuffman, and G. Raithel. Publisher's note: Level shifts of rubidium rydberg states due to binary interactions [phys. rev. a 75, 032712 (2007)]. Phys. Rev. A, 75(3):039902-+, March 2007.

[37] S. F. Yelin, M. Kostrun, T. Wang, and M. Fleischhauer. Correlation in superradiance: A closed-form approach to cooperative effects. ArXiv Quantum Physics e-prints, September 2005.

[38] P. G. Brooke, K.-P. Marzlin, J. D. Cresser, and B. C. Sanders. Superand subradiant emission of two-level systems in the near-dicke limit. Phys. Rev. A, 77(3):033844-+, March 2008.

[39] T. J. Carroll, K. Claringbould, A. Goodsell, M. J. Lim, and M. W. Noel. Angular dependence of the dipole-dipole interaction in a nearly one-dimensional sample of rydberg atoms. Physical Review Letters, 93(15):153001-+, October 2004. 
[40] I. Mourachko, W. Li, and T. F. Gallagher. Controlled many-body interactions in a frozen rydberg gas. Phys. Rev. A, 70(3):031401-+, September 2004 .

[41] Tetsuji Tokihiro, Yoshihiro Manabe, and Eiichi Hanamura. Superradiance of frenkel excitons with any degree of excitation prepared by a short-pulse laser. Phys. Rev. B, 51(12):7655-7668, Mar 1995.

[42] T. Castellani and A. Cavagna. Spin-glass theory for pedestrians. J. Stat. Mech, 5012:52, 2005.

[43] Th. F'orster. Zwischenmolekulare energiewanderung und fluoreszenz. $A n$ nalen der Physik, 437:55, 1948.

[44] J. Weiner, V. S. Bagnato, S. Zilio, and P. S. Julienne. Experiments and theory in cold and ultracold collisions. Reviews of Modern Physics, 71:185, January 1999.

[45] K. M. Jones, E. Tiesinga, P. D. Lett, and P. S. Julienne. Ultracold photoassociation spectroscopy: Long-range molecules and atomic scattering. Reviews of Modern Physics, 78:483-535, April 2006.

[46] G. K. Brennen, I. H. Deutsch, and P. S. Jessen. Entangling dipoledipole interactions for quantum logic with neutral atoms. Phys. Rev. A, 61(6):062309-+, June 2000.

[47] I. I. Ryabtsev, D. B. Tretyakov, and I. I. Beterov. Applicability of rydberg atoms to quantum computers. Journal of Physics B Atomic Molecular Physics, 38:421-+, January 2005.

[48] N. Schlosser, G. Reymond, I. Protsenko, and P. Grangier. Sub-poissonian loading of single atoms in a microscopic dipole trap. Nature, 411:10241027, June 2001.

[49] J. Beugnon, M. P. A. Jones, J. Dingjan, B. Darquié, G. Messin, A. Browaeys, and P. Grangier. Quantum interference between two single photons emitted by independently trapped atoms. Nature, 440:779-782, April 2006.

[50] C. Ates, T. Pohl, T. Pattard, and J. M. Rost. Many-body theory of excitation dynamics in an ultracold rydberg gas. Phys. Rev. A, 76(1):013413-+, July 2007.

[51] M. Reetz-Lamour, J. Deiglmayr, T. Amthor, and M. Weidemüller. Rabi oscillations between ground and rydberg states and van der waals blockade in a mesoscopic frozen rydberg gas. New Journal of Physics, 10(4):045026+ , April 2008. 
[52] T. A. Johnson, E. Urban, T. Henage, L. Isenhower, D. D. Yavuz, T. G. Walker, and M. Saffman. Rabi oscillations between ground and rydberg states with dipole-dipole atomic interactions. Physical Review Letters, 100(11):113003-+, March 2008.

[53] E. Urban, T. A. Johnson, T. Henage, L. Isenhower, D. D. Yavuz, T. G. Walker, and M. Saffman. Observation of rydberg blockade between two atoms. Nature Physics, 5:110-114, February 2009.

[54] T. Wilk, A. Gaëtan, C. Evellin, J. Wolters, Y. Miroshnychenko, P. Grangier, and A. Browaeys. Entanglement of Two Individual Neutral Atoms Using Rydberg Blockade. Physical Review Letters, 104(1):010502-+, January 2010.

[55] T. Pohl and P. R. Berman. Breaking the dipole blockade: Nearly resonant dipole interactions in few-atom systems. Physical Review Letters, 102(1):013004-+, January 2009.

[56] D. Tong, S. M. Farooqi, J. Stanojevic, S. Krishnan, Y. P. Zhang, R. Côté, E. E. Eyler, and P. L. Gould. Local blockade of rydberg excitation in an ultracold gas. Physical Review Letters, 93(6):063001-+, August 2004.

[57] V. Vuletic. Quantum networks: When superatoms talk photons. Nature Physics, 2:801-802, December 2006.

[58] J. Stanojevic and R. Côté. Many-body rabi oscillations of rydberg excitation in small mesoscopic samples. Phys. Rev. A, 80(3):033418-+, September 2009 .

[59] R. Heidemann, U. Raitzsch, V. Bendkowsky, B. Butscher, R. Löw, L. Santos, and T. Pfau. Evidence for coherent collective rydberg excitation in the strong blockade regime. Physical Review Letters, 99(16):163601-+, October 2007.

[60] A. Browaeys, A. Gaëtan, T. Wilk, C. Evellin, J. Wolters, Y. Miroshnychenko, P. Grangier, P. Pillet, D. Comparat, A. Chotia, and M. Viteau. Entanglement of two individual atoms using the rydberg blockade. ArXiv e-prints, October 2009.

[61] K. Singer, M. Reetz-Lamour, T. Amthor, L. G. Marcassa, and M. Weidemüller. Suppression of excitation and spectral broadening induced by interactions in a cold gas of rydberg atoms. Physical Review Letters, 93(16):163001-+, October 2004.

[62] Amodsen Chotia, Matthieu Viteau, Thibault Vogt, Daniel Comparat, and Pierre Pillet. Kinetic monte carlo modeling of dipole blockade in rydberg excitation experiment. New J. Phys., 10:045031, 2008. 
[63] J. V. Hernández and F. Robicheaux. Simulation of a strong van der waals blockade in a dense ultracold gas. Journal of Physics B Atomic Molecular Physics, 41:5301-+, February 2008.

[64] H. Weimer, R. Löw, T. Pfau, and H. P. Büchler. Quantum critical behavior in strongly interacting rydberg gases. Physical Review Letters, 101(25):250601-+, December 2008.

[65] R. Löw, H. Weimer, U. Krohn, R. Heidemann, V. Bendkowsky, B. Butscher, H. P. Büchler, and T. Pfau. Universal scaling in a strongly interacting rydberg gas. Phys. Rev. A, 80(3):033422-+, September 2009.

[66] T. C. Liebisch, A. Reinhard, P. R. Berman, and G. Raithel. Atom counting statistics in ensembles of interacting rydberg atoms. Physical Review Letters, 95(25):253002-+, December 2005.

[67] T. C. Liebisch, A. Reinhard, P. R. Berman, and G. Raithel. Erratum: Atom counting statistics in ensembles of interacting rydberg atoms [phys. rev. lett. 95, 253002 (2005)]. Physical Review Letters, 98(10):109903-+, March 2007.

[68] I. I. Ryabtsev, D. B. Tretyakov, I. I. Beterov, and V. M. Entin. Effect of finite detection efficiency on the observation of the dipole-dipole interaction of a few rydberg atoms. Phys. Rev. A, 76(1):012722-+, July 2007.

[69] I. I. Ryabtsev, D. B. Tretyakov, I. I. Beterov, and V. M. Entin. Erratum: Effect of finite detection efficiency on the observation of the dipoledipole interaction of a few rydberg atoms [phys. rev. a 76, 012722 (2007)]. Phys. Rev. A, 76(4):049902-+, October 2007.

[70] I. I. Ryabtsev, D. B. Tretyakov, I. I. Beterov, and V. M. Entin. Observation of the Stark-Tuned Förster Resonance between Two Rydberg Atoms. Physical Review Letters, 104(7):073003-+, February 2010.

[71] K. Afrousheh, P. Bohlouli-Zanjani, D. Vagale, A. Mugford, M. Fedorov, and J. D. Martin. Spectroscopic observation of resonant electric dipoledipole interactions between cold rydberg atoms. Physical Review Letters, 93(23):233001-+, November 2004.

[72] D. B. Tretyakov, I. I. Beterov, V. M. Entin, I. I. Ryabtsev, and P. L. Chapovsky. Investigation of cold rubidium rydberg atoms in a magnetooptical trap. Soviet Journal of Experimental and Theoretical Physics, 108:374-383, March 2009.

[73] A. Reinhard, K. C. Younge, T. C. Liebisch, B. Knuffman, P. R. Berman, and G. Raithel. Double-resonance spectroscopy of interacting rydbergatom systems. Physical Review Letters, 100(23):233201-+, June 2008. 
[74] C. Ates, T. Pohl, T. Pattard, and J. M. Rost. Antiblockade in rydberg excitation of an ultracold lattice gas. Physical Review Letters, 98(2):023002+ , January 2007.

[75] T. Amthor, C. Giese, C. S. Hofmann, and M. Weidemüller. Evidence of Antiblockade in an Ultracold Rydberg Gas. Physical Review Letters, 104(1):013001-+, January 2010.

[76] T. Vogt, M. Viteau, J. Zhao, A. Chotia, D. Comparat, and P. Pillet. Dipole blockade at förster resonances in high resolution laser excitation of rydberg states of cesium atoms. Physical Review Letters, 97(8):083003-+, August 2006.

[77] T. Vogt, M. Viteau, A. Chotia, J. Zhao, D. Comparat, and P. Pillet. Electric-field induced dipole blockade with rydberg atoms. Physical Review Letters, 99(7):073002-+, August 2007.

[78] B. Sun and F. Robicheaux. Spectral linewidth broadening from pair fluctuations in a frozen rydberg gas. Phys. Rev. A, 78(4):040701-+, October 2008.

[79] T. J. Carroll, C. Daniel, L. Hoover, T. Sidie, and M. W. Noel. Simulations of the dipole-dipole interaction between two spatially separated groups of Rydberg atoms. Phys. Rev. A, 80(5):052712-+, November 2009.

[80] C. S. E. van Ditzhuijzen, A. F. Koenderink, J. V. Hernández, F. Robicheaux, L. D. Noordam, and H. B. V. L. van den Heuvell. Spatially resolved observation of dipole-dipole interaction between rydberg atoms. Physical Review Letters, 100(24):243201-+, June 2008.

[81] A. Tauschinsky, C. S. E. van Ditzhuijzen, L. D. Noordam, and H. B. V. L. van den Heuvell. Radio-frequency-driven dipole-dipole interactions in spatially separated volumes. Phys. Rev. A, 78(6):063409-+, December 2008.

[82] P. Bohlouli-Zanjani, J. A. Petrus, and J. D. D. Martin. Enhancement of rydberg atom interactions using ac stark shifts. Physical Review Letters, 98(20):203005-+, May 2007.

[83] J. A. Petrus, P. Bohlouli-Zanjani, and J. D. D. Martin. ac electric-fieldinduced resonant energy transfer between cold rydberg atoms. Journal of Physics B Atomic Molecular Physics, 41(24):245001-+, December 2008.

[84] O. Kritsun, O. Boiko, and H. Metcalf. Excitation of metastable helium atoms to rydberg states by stirap. APS Meeting Abstracts, pages D1026+, May 2004.

[85] T. Cubel, B. K. Teo, V. S. Malinovsky, J. R. Guest, A. Reinhard, B. Knuffman, P. R. Berman, and G. Raithel. Coherent population transfer of ground-state atoms into rydberg states. Phys. Rev. A, 72(2):023405-+, August 2005. 
[86] K. J. Weatherill, J. D. Pritchard, R. P. Abel, M. G. Bason, A. K. Mohapatra, and C. S. Adams. Fast track communication: Electromagnetically induced transparency of an interacting cold rydberg ensemble. Journal of Physics B Atomic Molecular Physics, 41(20):201002-+, October 2008.

[87] H. Schempp, C. Giese, S. D. Saliba, C. S. Hofmann, G. Günter, B. D. DePaola, T. Amthor, M. Weidemüller, S. Sevincli, and T. Pohl. Coherent population trapping with controlled interparticle interactions. ArXiv eprints, December 2009.

[88] A. K. Mohapatra, T. R. Jackson, and C. S. Adams. Coherent optical detection of highly excited rydberg states using electromagnetically induced transparency. Physical Review Letters, 98(11):113003-+, March 2007.

[89] W. R. Anderson, M. P. Robinson, J. D. Martin, and T. F. Gallagher. Dephasing of resonant energy transfer in a cold rydberg gas. Phys. Rev. A, 65(6):063404-+, June 2002.

[90] M. Mudrich, N. Zahzam, T. Vogt, D. Comparat, and P. Pillet. Back and forth transfer and coherent coupling in a cold rydberg dipole gas. Physical Review Letters, 95(23):233002-+, November 2005.

[91] U. Raitzsch, V. Bendkowsky, R. Heidemann, B. Butscher, R. Löw, and T. Pfau. Echo experiments in a strongly interacting rydberg gas. Physical Review Letters, 100(1):013002-+, January 2008.

[92] U. Raitzsch, R. Heidemann, H. Weimer, B. Butscher, P. Kollmann, R. Löw, H. P. Büchler, and T. Pfau. Investigation of dephasing rates in an interacting rydberg gas. New Journal of Physics, 11(5):055014-+, May 2009.

[93] J. V. Hernández and F. Robicheaux. Simulations using echo sequences to observe coherence in a cold rydberg gas. Journal of Physics B Atomic Molecular Physics, 41(19):195301-+, October 2008.

[94] K. C. Younge and G. Raithel. Rotary echo tests of coherence in rydbergatom excitation. New Journal of Physics, 11(4):043006-+, April 2009.

[95] S. Wüster, J. Stanojevic, C. Ates, T. Pohl, P. Deuar, J. F. Corney, and J. M. Rost. Correlations of Rydberg excitations in an ultracold gas after an echo sequence. Phys. Rev. A, 81(2):023406-+, February 2010.

[96] N. Saquet, A. Cournol, J. Beugnon, J. Robert, P. Pillet, and N. Vanhaecke. Landau-zener transitions in frozen pairs of rydberg atoms. 2010.

[97] C. S. E. van Ditzhuijzen, A. Tauschinsky, and H. B. van Linden van den Heuvell. Observation of Stückelberg oscillations in dipole-dipole interactions. Phys. Rev. A, 80(6):063407-+, December 2009. 
[98] T. Wang, S. F. Yelin, R. Côté, E. E. Eyler, S. M. Farooqi, P. L. Gould, M. Koštrun, D. Tong, and D. Vrinceanu. Superradiance in ultracold rydberg gases. Phys. Rev. A, 75(3):033802-+, March 2007.

[99] J. O. Day, E. Brekke, and T. G. Walker. Dynamics of low-density ultracold rydberg gases. Phys. Rev. A, 77(5):052712-+, May 2008.

[100] P. Maioli, T. Meunier, S. Gleyzes, A. Auffeves, G. Nogues, M. Brune, J. M. Raimond, and S. Haroche. Nondestructive rydberg atom counting with mesoscopic fields in a cavity. Physical Review Letters, 94(11):113601-+, March 2005.

[101] S. Haroche, M. Brune, and J.-M. Raimond. Schrödinger cat states and decoherence studies in cavity qed. The European Physical Journal Special Topics, Volume 159, Issue 1, pp.19-26, 159:19-26, June 2008.

[102] E. Brekke, J. O. Day, and T. G. Walker. Four-wave mixing in ultracold atoms using intermediate rydberg states. Phys. Rev. A, 78(6):063830-+, December 2008.

[103] L. Santos, G. V. Shlyapnikov, P. Zoller, and M. Lewenstein. Bose-einstein condensation in trapped dipolar gases. Physical Review Letters, 85:17911794, August 2000.

[104] M. Baranov, Ł. G. K. Dobrek, K. Góral, L. Santos, and M. Lewenstein. Ultracold dipolar gases - a challenge for experiments and theory. Physica Scripta Volume T, 102:74-81, 2002.

[105] T Lahaye, C Menotti, L Santos, M Lewenstein, and T Pfau. The physics of dipolar bosonic quantum gases. Rep. Prog. Phys., 72:126401, 2009.

[106] L. Santos and T. Pfau. Spin-3 chromium bose-einstein condensates. Physical Review Letters, 96(19):190404-+, May 2006.

[107] Y. Kawaguchi, H. Saito, and M. Ueda. Einstein de haas effect in dipolar bose-einstein condensates. Physical Review Letters, 96(8):080405-+, March 2006.

[108] Roman V Krems. Cold controlled chemistry. Phys. Chem. Chem. Phys., 2008.

[109] Martin T. Bell and Timothy P. Softley. Ultracold molecules and ultracold chemistry. Molecular Physics, 107(2):99, 2009.

[110] L.D. Carr, D. DeMille, R.V. Krems, and J. Ye. Cold and ultracold molecules: science, technology and applications. New Journal of Physics, 11(055049):055049, 2009.

[111] O. Dulieu and C. Gabbanini. The formation and interactions of cold and ultracold molecules: new challenges for interdisciplinary physics. Reports on Progress in Physics, 72(8):086401-+, August 2009. 
[112] G. Pupillo, A. Micheli, M. Boninsegni, I. Lesanovsky, and P. Zoller. Mesoscopic phases of dipolar ensembles with polar molecules and rydberg atoms. ArXiv e-prints, April 2009.

[113] J. Stuhler, A. Griesmaier, T. Koch, M. Fattori, T. Pfau, S. Giovanazzi, P. Pedri, and L. Santos. Observation of dipole-dipole interaction in a degenerate quantum gas. Physical Review Letters, 95(15):150406-+, October 2005 .

[114] T. Koch, T. Lahaye, J. Metz, B. Fröhlich, A. Griesmaier, and T. Pfau. Stabilization of a purely dipolar quantum gas against collapse. Nature Physics, 4:218-222, March 2008.

[115] G. Pupillo, A. Micheli, M. Boninsegni, I. Lesanovsky, and P. Zoller. Strongly correlated gases of rydberg-dressed atoms: quantum and classical dynamics. 2010.

[116] R. Heidemann, U. Raitzsch, V. Bendkowsky, B. Butscher, R. Löw, and T. Pfau. Rydberg excitation of bose-einstein condensates. Physical Review Letters, 100(3):033601-+, January 2008.

[117] J. H. Wesenberg, K. Mølmer, L. Rippe, and S. Kröll. Scalable designs for quantum computing with rare-earth-ion-doped crystals. Phys. Rev. A, 75(1):012304-+, January 2007.

[118] J. S. Frasier, V. Celli, and T. Blum. Resonant processes in a frozen gas. Phys. Rev. A, 59:4358-4367, June 1999.

[119] F. Robicheaux, J. V. Hernández, T. Topçu, and L. D. Noordam. Simulation of coherent interactions between rydberg atoms. Phys. Rev. A, 70(4):042703-+, October 2004.

[120] F. Robicheaux and J. V. Hernández. Many-body wave function in a dipole blockade configuration. Phys. Rev. A, 72(6):063403, Dec 2005.

[121] S. Westermann, T. Amthor, A. L. de Oliveira, J. Deiglmayr, M. ReetzLamour, and M. Weidemüller. Dynamics of resonant energy transfer in a cold rydberg gas. European Physical Journal D, 40:37-43, October 2006.

[122] K. C. Younge, A. Reinhard, T. Pohl, P. R. Berman, and G. Raithel. Mesoscopic rydberg ensembles: Beyond the pairwise-interaction approximation. Phys. Rev. A, 79(4):043420-+, April 2009.

[123] M. Reetz-Lamour, T. Amthor, S. Westermann, J. Denskat, A. L. de Oliveira, and M. Weidemüller. Modeling few-body phenomena in an ultracold rydberg gas. Nuclear Physics A, 790:728-732, June 2007.

[124] W. Li, P. J. Tanner, Y. Jamil, and T. F. Gallagher. Ionization and plasma formation in high n cold rydberg samples. European Physical Journal D, 40:27-35, October 2006. 
[125] T C Killian, V S Ashoka, P Gupta, S Laha, S B Nagel, C E Simien, S Kulin, S L Rolston, and S D Bergeson. Ultracold neutral plasmas: recent experiments and new prospect. J. Phys. A: Math. Gen., 36:6077, may 2003.

[126] T C Killian, T Pattard, T Pohl, and J M Rost. Ultracold neutral plasmas. Physics Reports, 449:77-130, 2007.

[127] F. Robicheaux. Ionization due to the interaction between two rydberg atoms. Journal of Physics B Atomic Molecular Physics, 38:333-+, January 2005.

[128] T. C. Killian, M. J. Lim, S. Kulin, R. Dumke, S. D. Bergeson, and S. L. Rolston. Formation of rydberg atoms in an expanding ultracold neutral plasma. Phys. Rev. Lett., 86(17):3759, 2001.

[129] T. F. Gallagher, P. Pillet, M. P. Robinson, B. Laburthe-Tolra, and M. W. Noel. Back and forth between rydberg atoms and ultracold plasmas. Journal of the Optical Society of America B Optical Physics, 20:1091-1097, May 2003.

[130] D. Comparat, T. Vogt, N. Zahzam, M. Mudrich, and P. Pillet. Star cluster dynamics in a laboratory: electrons in an ultracold plasma. MNRAS, 361:1227-1242, August 2005.

[131] A. Walz-Flannigan, J. R. Guest, J.-H. Choi, and G. Raithel. Cold-rydberggas dynamics. Phys. Rev. A, 69:063405, 2004.

[132] W. Li, andM.P. Robinson M.W. Noel, P.J. Tanner, T.F. Gallagher, D. Comparat, B. Laburthe-Tolra, N. Vanhaecke, T. Vogt, N. Zahzam, P. Pillet, and D. A. Tate. Evolution dynamics of a dense, frozen rydberg gas to plasma. Phys. Rev. A, 70:042713, 2004.

[133] W. Li, P. J. Tanner, and T. F. Gallagher. Dipole-dipole excitation and ionization in an ultracold gas of rydberg atoms. Physical Review Letters, 94(17):173001-+, May 2005.

[134] B. Knuffman and G. Raithel. Emission of fast atoms from a cold rydberg gas. Phys. Rev. A, 73(2):020704-+, February 2006.

[135] V. A. Nascimento, L. L. Caliri, A. Schwettmann, J. P. Shaffer, and L. G. Marcassa. Electric field effects in the excitation of cold rydberg-atom pairs. Physical Review Letters, 102(21):213201-+, May 2009.

[136] T. Amthor, M. Reetz-Lamour, S. Westermann, J. Denskat, and M. Weidemüller. Mechanical effect of van der waals interactions observed in real time in an ultracold rydberg gas. Physical Review Letters, 98(2):023004+, January 2007. 
[137] T. Amthor, M. Reetz-Lamour, C. Giese, and M. Weidemüller. Modeling many-particle mechanical effects of an interacting rydberg gas. Phys. Rev. A, 76(5):054702-+, November 2007.

[138] T. Amthor, J. Denskat, C. Giese, N. N. Bezuglov, A. Ekers, L. S. Cederbaum, and M. Weidemüller. Autoionization of an ultracold rydberg gas through resonant dipole coupling. European Physical Journal D, 53:329335 , June 2009.

[139] M. Viteau, A. Chotia, D. Comparat, D. A. Tate, T. F. Gallagher, and P. Pillet. Melting a frozen rydberg gas with an attractive potential. Phys. Rev. A, 78(4):040704-+, October 2008.

[140] C. Ates, A. Eisfeld, and J. M. Rost. Motion of rydberg atoms induced by resonant dipole dipole interactions. New Journal of Physics, 10(4):045030+ , April 2008.

[141] Yukap Hahn. Density dependence of molecular autoionization in a cold gas. J. Phys. B, 33:L655, 2000.

[142] M. Viteau, A. Chotia, D. Comparat, D. Tate, T. F. Gallagher, and P. Pillet. Melting a frozen rydberg gas with an attractive potential. Phys. Rev. A, 78:040704, 2008.

[143] C. H. Greene, A. S. Dickinson, and H. R. Sadeghpour. Creation of polar and nonpolar ultra-long-range rydberg molecules. Physical Review Letters, 85:2458-2461, September 2000.

[144] B. E. Granger, E. L. Hamilton, and C. H. Greene. Quantum and semiclassical analysis of long-range rydberg molecules. Phys. Rev. A, 64(4):042508-+, October 2001.

[145] E. L. Hamilton, C. H. Greene, and H. R. Sadeghpour. Letter to the editor: Shape-resonance-induced long-range molecular rydberg states. Journal of Physics B Atomic Molecular Physics, 35:L199-L206, May 2002.

[146] S. M. Farooqi, D. Tong, S. Krishnan, J. Stanojevic, Y. P. Zhang, J. R. Ensher, A. S. Estrin, C. Boisseau, R. Côté, E. E. Eyler, and P. L. Gould. Long-range molecular resonances in a cold rydberg gas. Physical Review Letters, 91(18):183002-+, October 2003.

[147] A. L. de Oliveira, M. W. Mancini, V. S. Bagnato, and L. G. Marcassa. Rydberg cold collisions dominated by ultralong range potential. Physical Review Letters, 90(14):143002-+, April 2003.

[148] C. H. Greene, E. L. Hamilton, H. Crowell, C. Vadla, and K. Niemax. Experimental verification of minima in excited long-range rydberg states of $\mathrm{rb}_{2}$. Physical Review Letters, 97(23):233002-+, December 2006. 
[149] C. Vadla, V. Horvatic, and K. Niemax. Line-broadening studies of excited diatomic homoatomic and heteroatomic rydberg molecules formed by potassium, rubidium, and cesium atoms. Phys. Rev. A, 80(5):052506+ , November 2009.

[150] V. Bendkowsky, B. Butscher, J. Nipper, J. P. Shaffer, R. Löw, and T. Pfau. Observation of ultralong-range rydberg molecules. Nature, 458:1005-1008, April 2009.

[151] V. Bendkowsky, B. Butscher, J. Nipper, J. Balewski, J. P. Shaffer, R. Löw, T. Pfau, W. Li, J. Stanojevic, T. Pohl, and J. M. Rost. Rydberg trimers and excited dimers bound by internal quantum reflection. ArXiv e-prints, December 2009.

[152] C. Boisseau, I. Simbotin, and R. Côté. Macrodimers: Ultralong range rydberg molecules. Physical Review Letters, 88(13):133004-+, April 2002.

[153] M. I. Chibisov, A. A. Khuskivadze, and I. I. Fabrikant. Letter to the editor: Energies and dipole moments of long-range molecular rydberg states. Journal of Physics B Atomic Molecular Physics, 35:L193-L198, May 2002.

[154] A. A. Khuskivadze, M. I. Chibisov, and I. I. Fabrikant. Adiabatic energy levels and electric dipole moments of rydberg states of $\mathrm{rb}_{2}$ and $\mathrm{cs}_{2}$ dimers. Phys. Rev. A, 66(4):042709-+, October 2002.

[155] I. C. H. Liu and J. M. Rost. Polyatomic molecules formed with a rydberg atom in an ultracold environment. European Physical Journal D, 40:65-71, October 2006.

[156] M. Mayle, B. Hezel, I. Lesanovsky, and P. Schmelcher. One-dimensional rydberg gas in a magnetoelectric trap. Physical Review Letters, 99(11):113004-+, September 2007.

[157] A. Schwettmann, J. Crawford, K. R. Overstreet, and J. P. Shaffer. Cold cs rydberg-gas interactions. Phys. Rev. A, 74(2):020701-+, August 2006.

[158] I. C. H. Liu, J. Stanojevic, and J. M. Rost. Ultra-long-range rydberg trimers with a repulsive two-body interaction. Physical Review Letters, 102(17):173001-+, May 2009.

[159] K. R. Overstreet, A. Schwettmann, J. Tallant, D. Booth, and J. P. Shaffer. Observation of electric-field-induced cs rydberg atom macrodimers. Nature Physics, 5:581-585, August 2009.

[160] K. R. Overstreet, A. Schwettmann, J. Tallant, and J. P. Shaffer. Photoinitiated collisions between cold cs rydberg atoms. Phys. Rev. A, 76(1):011403-+, July 2007. 
[161] M. Müller, I. Lesanovsky, H. Weimer, H. P. Büchler, and P. Zoller. Mesoscopic rydberg gate based on electromagnetically induced transparency. Physical Review Letters, 102(17):170502-+, May 2009.

[162] H. Weimer, M. Müller, I. Lesanovsky, P. Zoller, and H. P. Büchler. A Rydberg quantum simulator. Nature Physics, 6:382-388, May 2010.

[163] S. I. Schmid and J. Evers. Dipole-dipole interaction between orthogonal dipole moments in time-dependent geometries. Phys. Rev. A, 77(1):013822-+, January 2008.

[164] M. Müller, L. Liang, I. Lesanovsky, and P. Zoller. Trapped rydberg ions: from spin chains to fast quantum gates. New Journal of Physics, 10(9):093009-+, September 2008.

[165] B. Olmos, R. González-Férez, and I. Lesanovsky. Collective rydberg excitations of an atomic gas confined in a ring lattice. Phys. Rev. A, 79(4):043419-+, April 2009.

[166] B. Olmos, R. González-Férez, and I. Lesanovsky. Fermionic collective excitations in a lattice gas of rydberg atoms. Physical Review Letters, 103(18):185302-+, October 2009.

[167] R. Côté, A. Russell, E.E. Eyler, and P.L. Gould. Quantum random walk with rydberg atoms in an optical lattice. New Journal of Physics, 8:156, 2006.

[168] B. Vaucher, S. J. Thwaite, and D. Jaksch. Ultralarge rydberg dimers in optical lattices. Phys. Rev. A, 78(4):043415-+, October 2008.

[169] M. L. Wall, F. Robicheaux, and R. R. Jones. Controlling atom motion through the dipole dipole force. Journal of Physics B Atomic Molecular Physics, 40:3693-3705, September 2007.

[170] T. Pohl, E. Demler, and M. D. Lukin. Dynamical crystallization in the dipole blockade of ultracold atoms. ArXiv e-prints, November 2009.

[171] J. Qian, Y. Qian, M. Ke, X.-L. Feng, C. H. Oh, and Y. Wang. Breakdown of the dipole blockade with a zero-area phase-jump pulse. Phys. Rev. A, 80(5):053413-+, November 2009.

[172] T. Pohl, T. Pattard, and J. M. Rost. Coulomb crystallization in expanding laser-cooled neutral plasmas. Physical Review Letters, 92(15):155003-+, April 2004.

[173] T. Pohl, T. Pattard, and J. M. Rost. Letter to the editor: On the possibility of 'correlation cooling' of ultracold neutral plasmas. Journal of Physics B Atomic Molecular Physics, 37:L183-L191, May 2004. 
[174] A. A. Svidzinsky, J.-T. Chang, and M. O. Scully. Cooperative spontaneous emission of $\mathrm{N}$ atoms: Many-body eigenstates, the effect of virtual Lamb shift processes, and analogy with radiation of $\mathrm{N}$ classical oscillators. Phys. Rev. A, 81(5):053821-+, May 2010. 


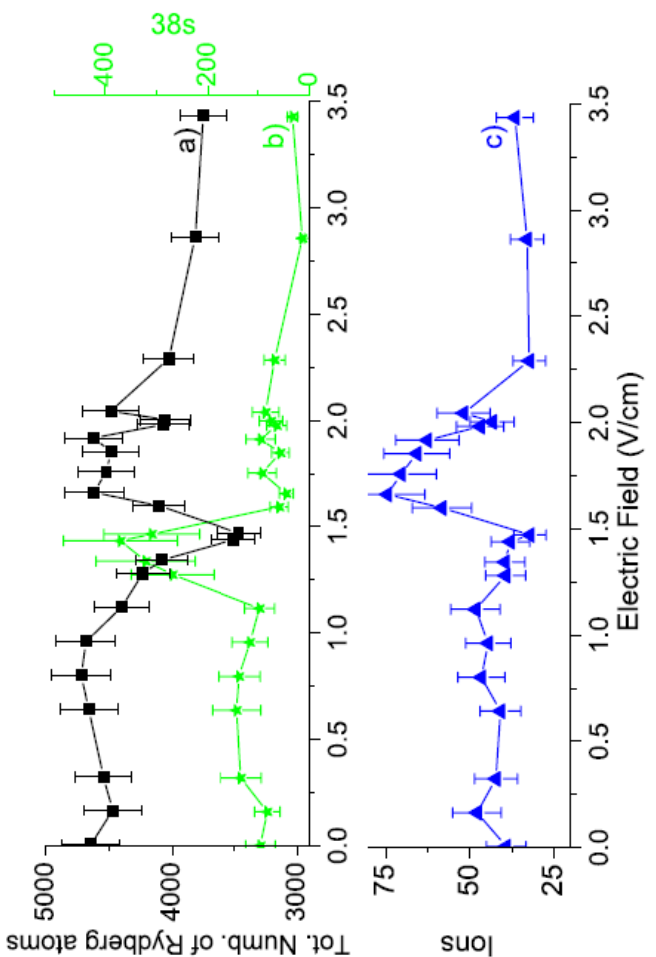




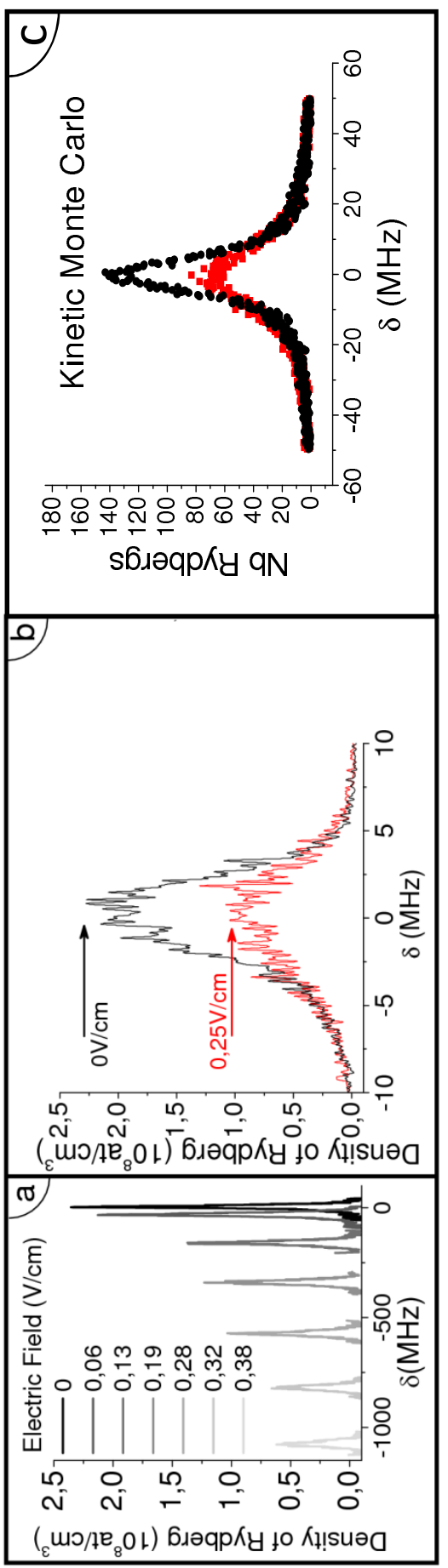

